\title{
A FUNCTORIAL EXTENSION OF THE ABELIAN REIDEMEISTER TORSIONS OF THREE-MANIFOLDS
}

\author{
VINCENT FLORENS AND GWÉNAËL MASSUYEAU
}

\begin{abstract}
Let $\mathbb{F}$ be a field and let $G \subset \mathbb{F} \backslash\{0\}$ be a multiplicative subgroup. We consider the category $\mathrm{Cob}_{G}$ of 3 -dimensional cobordisms equipped with a representation of their fundamental group in $G$, and the category $\operatorname{Vect}_{\mathbb{F}, \pm G}$ of $\mathbb{F}$-linear maps defined up to multiplication by an element of $\pm G$. Using the elementary theory of Reidemeister torsions, we construct a "Reidemeister functor" from Cob $_{G}$ to $\mathrm{Vect}_{\mathbb{F}, \pm G}$. In particular, when the group $G$ is free abelian and $\mathbb{F}$ is the field of fractions of the group ring $\mathbb{Z}[G]$, we obtain a functorial formulation of an Alexander-type invariant introduced by Lescop for 3-manifolds with boundary; when $G$ is trivial, the Reidemeister functor specializes to the TQFT developed by Frohman and Nicas to enclose the Alexander polynomial of knots. The study of the Reidemeister functor is carried out for any multiplicative subgroup $G \subset \mathbb{F} \backslash\{0\}$. We obtain a duality result and we show that the resulting projective representation of the monoid of homology cobordisms is equivalent to the Magnus representation combined with the relative Reidemeister torsion.
\end{abstract}

\section{Contents}

1. Introduction

2. The Alexander functor A $r$

3. Alexander functor and knots 10

4. The Reidemeister functor R 12

5. Back to the Alexander functor $\quad 18$

6. Reidemeister functor and knots 20

7. The monoid of homology cobordisms 23

8. Computations with Heegaard splittings 26

9. Duality 28

Appendix A. A short review of combinatorial torsions 33

References

\section{INTRODUCTION}

Let Cob be the category of 3-dimensional cobordisms introduced by Crane and Yetter [CY99], and whose definition we briefly recall. The objects of Cob are integers $g \geq 0$, and correspond to compact connected oriented surfaces $F_{g}$ of genus $g$ with one boundary component. Indeed, we fix for every $g \geq 0$ a model surface $F_{g}$ whose boundary is identified with $S^{1}$, and we also fix a base point $\star$ on $\partial F_{g}=S^{1}$. The morphisms $g_{-} \rightarrow g_{+}$ in the category Cob are the equivalence classes of cobordisms between the surfaces $F_{g_{-}}$and $F_{g_{+}}$. To be more specific, a cobordism from $F_{g_{-}}$to $F_{g_{+}}$is a pair $(M, m)$ consisting of a compact connected oriented 3-manifold $M$ and an orientation-preserving homeomorphism $m: F\left(g_{-}, g_{+}\right) \rightarrow \partial M$ where

$$
F\left(g_{-}, g_{+}\right):=-F_{g_{-}} \cup_{S^{1} \times\{-1\}}\left(S^{1} \times[-1,1]\right) \cup_{S^{1} \times\{1\}} F_{g_{+}} ;
$$

Date: October 23, 2014. 
two such pairs $(M, m)$ and $\left(M^{\prime}, m^{\prime}\right)$ are equivalent if there exists a homeomorphism $f: M \rightarrow M^{\prime}$ such that $m^{\prime}=\left.f\right|_{\partial M} \circ m$. We shall denote a pair $(M, m)$ simply by the upper-case letter $M$, with the convention that the boundary-parametrization is always denoted by the lower-case letter $m$; besides, we denote by $m_{ \pm}: F_{g_{ \pm}} \rightarrow M$ the restriction of $m$ composed with the inclusion of $\partial M$ into $M$. Thus the cobordism $M$ "runs" from the bottom surface $\partial_{-} M:=m_{-}\left(F_{g_{-}}\right)$to the top surface $\partial_{+} M:=m_{+}\left(F_{g_{+}}\right)$. The degree of the cobordism $M$ is the integer $g_{+}-g_{-}$.

The composition $N \circ M$ of two cobordisms $M, N$ in Cob is defined by identifying $\partial_{+} M$ to $\partial_{-} N$ and, for any integer $g \geq 0$, the identity of the object $g$ is the cylinder $F_{g} \times[-1,1]$ with the obvious boundary-parametrization. Our model surfaces $F_{0}, F_{1}, F_{2}, \ldots$ also come with an identification of the boundary-connected sum $F_{g} \sharp_{\partial} F_{h}$ with the surface $F_{g+h}$ for any $g, h \geq 0$. Thus the category Cob is enriched with a monoidal structure $\otimes$ : the tensor product $g \otimes h$ of two integers $g, h$ is the sum $g+h$, and the tensor product $M \otimes N$ of two cobordisms $M, N$ is their boundary-connected sum $M \sharp_{\partial} N$.

Let now $G$ be an abelian group. The category Cob can be refined to the category $\mathrm{Cob}_{G}$ of cobordisms equipped with a representation of the first integral homology group in $G$. To be more specific, an object of $\operatorname{Cob}_{G}$ is a pair $(g, \varphi)$ consisting of an integer $g \geq 0$ and a group homomorphism $\varphi: H_{1}\left(F_{g} ; \mathbb{Z}\right) \rightarrow G$. A morphism $\left(g_{-}, \varphi_{-}\right) \rightarrow\left(g_{+}, \varphi_{+}\right)$in the category $\operatorname{Cob}_{G}$ is a pair $(M, \varphi)$ where $M \in \operatorname{Cob}\left(g_{-}, g_{+}\right)$and $\varphi: H_{1}(M ; \mathbb{Z}) \rightarrow G$ is a group homomorphism such that $\varphi \circ m_{ \pm, *}=\varphi_{ \pm}$. The composition of two morphisms $(M, \varphi) \in \operatorname{Cob}_{G}\left(\left(g_{-}, \varphi_{-}\right),\left(g_{+}, \varphi_{+}\right)\right)$and $(N, \psi) \in \operatorname{Cob}_{G}\left(\left(h_{-}, \psi_{-}\right),\left(h_{+}, \psi_{+}\right)\right)$, such that $\left(g_{+}, \varphi_{+}\right)=\left(h_{-}, \psi_{-}\right)$, is defined by

$$
(N, \psi) \circ(M, \varphi):=(N \circ M, \psi+\varphi)
$$

where $N \circ M$ is the composition in Cob and $\psi+\varphi: H_{1}(N \circ M ; \mathbb{Z}) \rightarrow G$ is defined from $\varphi$ and $\psi$ by using the Mayer-Vietoris theorem. The monoidal structure of Cob also extends to the category $\mathrm{Cob}_{G}$ : the tensor product of objects is

$$
(g, \varphi) \otimes(h, \psi):=(g+h, \varphi \oplus \psi)
$$

where $H_{1}\left(F_{g+h} ; \mathbb{Z}\right)=H_{1}\left(F_{g} \sharp_{\partial} F_{h} ; \mathbb{Z}\right)$ is identified with $H_{1}\left(F_{g} ; \mathbb{Z}\right) \oplus H_{1}\left(F_{h} ; \mathbb{Z}\right)$, and the tensor product of morphisms is

$$
(M, \varphi) \otimes(N, \psi):=\left(M \sharp_{\partial} N, \varphi \oplus \psi\right)
$$

where $H_{1}\left(M \sharp_{\partial} N ; \mathbb{Z}\right)$ is identified with $H_{1}(M ; \mathbb{Z}) \oplus H_{1}(N ; \mathbb{Z})$.

Consider now a commutative ring $R$ and fix a subgroup $G \subset R^{\times}$of its group of units. Let $\operatorname{grMod}_{R, \pm G}$ be the category whose objects are $\mathbb{Z}$-graded $R$-modules and whose morphisms are graded $R$-linear maps of arbitrary degree, up to multiplication by an element of $\pm G$. The usual tensor product of graded $R$-modules defines a monoidal structure on the category $\operatorname{grMod}_{R, \pm G}$ : here the tensor product $a \otimes b$ of two graded $R$-linear maps $a: U \rightarrow U^{\prime}$ and $b: V \rightarrow V^{\prime}$ is defined with Koszul's rule, i.e. we set $(a \otimes b)(u \otimes v):=(-1)^{|b||u|} a(u) \otimes b(v)$ for any homogeneous elements $u \in U, v \in V$. In this paper, we construct and study two functors from $\operatorname{Cob}_{G}$ to $\operatorname{grMod}_{R, \pm G}$ for some specific rings $R$ and specific subgroups $G \subset R^{\times}$.

Our first functor is based on the "Alexander function" introduced by Lescop [Les98]. For any compact orientable 3-manifold $M$ with boundary, this function is defined on an exterior power of the Alexander module of $M$ relative to a boundary point, and it takes values in a ring of Laurent polynomials. Lescop's definition proceeds in a rather elementary way using a presentation of the Alexander module. 
Theorem I. Let $G$ be a finitely generated free abelian group, and let $\mathbb{Z}[G]$ be its group ring. Then there is a degree-preserving monoidal functor

$$
\mathrm{A}:=\mathrm{A}_{G}: \operatorname{Cob}_{G} \longrightarrow \operatorname{grMod}_{\mathbb{Z}[G], \pm G}
$$

which, at the level of objects, assigns to any $(g, \varphi)$ the exterior algebra of the $\varphi$-twisted relative homology group of the pair $\left(F_{g}, \star\right)$.

The $\mathbb{Z}[G]$-linear map $\mathrm{A}(M, \varphi)$ associated to a morphism $(M, \varphi)$ of $\operatorname{Cob}_{G}$ is defined in a very simple way from the Alexander function of $M$ using the decomposition of $\partial M$ into two parts, $\partial_{-} M$ and $\partial_{+} M$. The fact that the Alexander function gives rise to a functor on the category of cobordisms is somehow implicit in [Les98], where Lescop studies the behaviour of her invariant under some specific gluing operations. As it contains the Alexander polynomial of knots in a natural way, we call A the Alexander functor.

Since the works of Milnor [Mil62] and Turaev [Tur75], it is known that the Alexander polynomial of knots and 3-manifolds can be interpreted as a special kind of abelian Reidemeister torsion. We follow this direction to define our second functor, which we call the Reidemeister functor. In the sequel, the category $\operatorname{grMod}_{R, \pm G}$ associated to a field $R:=\mathbb{F}$ and a subgroup $G$ of $\mathbb{F}^{\times}=\mathbb{F} \backslash\{0\}$ is denoted by $\operatorname{grVect}_{\mathbb{F}, \pm G}$.

Theorem II. Let $\mathbb{F}$ be a field and let $G$ be a subgroup of $\mathbb{F}^{\times}$. Then there is a degreepreserving monoidal functor

$$
\mathrm{R}:=\mathrm{R}_{\mathbb{F}, G}: \operatorname{Cob}_{G} \longrightarrow \operatorname{grVect}_{\mathbb{F}, \pm G}
$$

which, at the level of objects, assigns to any $(g, \varphi)$ the exterior algebra of the $\varphi$-twisted relative homology group of the pair $\left(F_{g}, \star\right)$.

The construction of the functor R uses the elementary theory of Reidemeister torsions, but note that we need to consider cell chain complexes which are not necessarily acyclic. When $G$ is a finitely generated free abelian group and $\mathbb{F}:=Q(G)$ is the field of fractions of $\mathbb{Z}[G]$, we recover the functor A by extension of scalars. Thus it suffices to study the functor $\mathrm{R}$ and this is done using basic properties of combinatorial torsions. For instance, we compute its restriction to the monoid of homology cobordisms (which includes the mapping class group of a surface): we find that the representation induced by $\mathrm{R}$ is equivalent to the Magnus representation combined with the Reidemeister torsion of cobordisms relative to the top surface. We also give a formula for R in terms of Heegaard splittings and we show that $\mathrm{R}$ satisfies some duality properties, which generalize the symmetry properties of the Alexander polynomial of knots and 3-manifolds.

It is expected that Turaev's refinements of the Reidemeister torsion [Tur86, Tur89] can be adapted to refine R to a kind of "monoidal" degree-preserving functor from Cob $_{G}$ to the category $\operatorname{grVect}_{\mathbb{F}}$ of graded $\mathbb{F}$-vector spaces: the sign ambiguity would presumably be fixed using homological orientations on the manifolds, while the ambiguity in $G$ would be fixed by adding Euler structures. (Observe however that, since we use Koszul's rule and we allow morphisms in grVect $_{\mathbb{F}}$ to have non-zero degree, this category is not monoidal in the usual sense of the word.)

We now explain how our constructions are related to prior works. Soon after the emergence of quantum invariants of 3-manifolds in the late eighties, there have been several works which showed how to interpret the classical Alexander polynomial in this new framework. A more general problem was then to extend the Alexander polynomial to a functor from a category of cobordisms to a category of vector spaces following, as close as possible, the axioms of a TQFT [Ati88]. This problem has been solved by Frohman and Nicas who used elementary intersection theory in U(1)-representation 
varieties of surfaces [FN91]. (See also [FN94] for a much more general construction using $\mathrm{PU}(N)$-representations.) Later, Kerler showed that the Frohman-Nicas functor is in fact equivalent to a TQFT based on a certain quasitriangular Hopf algebra [Ker03a]. The Alexander polynomial of a knot $K$ in an integral homology 3 -sphere $N$ is recovered from this functor by taking the "graded" trace of the endomorphism associated to the cobordism that one obtains by "cutting" $N \backslash K$ along a Seifert surface of $K$. It turns out that, in the case $G=\{1\}$, the Alexander functor A is equivalent to the Frohman-Nicas functor. Note that the way how their functor determines the Alexander polynomial is somehow extrinsic, in that it goes through the choice of a Seifert surface. On the contrary, the functor A for $G=\mathbb{Z}$ intrinsically contains the Alexander polynomial of oriented knots in oriented integral homology 3-spheres by considering any knot of this type as a "bottom knot" in the style of [Hab06], i.e. by regarding its exterior as a morphism $1 \rightarrow 0$ in $\mathrm{Cob}_{G}$. Since this functorial extension of the Alexander polynomial applies to cobordisms $M$ equipped with an element of $H^{1}(M ; \mathbb{Z})$, it should be regarded as a kind of HQFT with target $\mathrm{K}(\mathbb{Z}, 1)$ - see [Tur10] - rather than a TQFT.

Our constructions are also related to the work of Bigelow, Cattabriga and the first author [BCF12], which provides a functorial extension of the Alexander polynomial to the category of tangles instead of the category of cobordisms. To describe this relation, let TangCob be the monoidal category whose objects are pairs of non-negative integers $(g, n)$ - corresponding to surfaces $F_{g}$ with $n$ punctures - and whose morphisms are cobordisms with tangles inside. Clearly the category TangCob contains the category Cob of [CY99] as well as the usual category Tang of (unoriented) tangles in the standard ball; for any abelian group $G$, there is an obvious refinement $\operatorname{Tang} \operatorname{Cob}_{G}$ of the category TangCob. When $G$ is the infinite cyclic group generated by $t$, the usual category Tang of oriented tangles in the standard ball can be regarded as a subcategory of TangCob ${ }_{G}$ by only considering those representations of tangle exteriors that send any oriented meridian to the generator $t$. The functors $\mathrm{A}$ and $\mathrm{R}$ constructed in this paper could be extended to the category TangCob ${ }_{G}$ using similar methods, but with more technicality. When $G$ is infinite cyclic, the restriction of the resulting functor A : TangCob $\operatorname{CrMod}_{\mathbb{Z}[G], \pm G}$ to Tang + would coincide with the "Alexander representation of tangles" constructed in [BCF12]. We also mention Archibald's extension of the Alexander polynomial [Arc10], which is based on diagrammatic presentations of tangles: her invariant seems to be very close to the invariant constructed in [BCF12] and it is stronger since it is defined without ambiguity in $\pm G$.

Finally, our approach is related to the work of Cimasoni and Turaev on "Lagrangian representations of tangles" [CT05, CT06]. These representations are functors from the category Tang + to the category of "Lagrangian relations" (which generalizes the category of $\mathbb{Z}\left[t^{ \pm 1}\right]$-modules equipped with non-degenerate skew-hermitian forms) and, for string links, they are equivalent to the (reduced) Burau representation [LD92, KLW01]. The constructions of [CT05, CT06] could be adapted to the case of cobordisms in order to obtain a functor from $\operatorname{Cob}_{G}$ to the category of "Lagrangian relations" over the $\operatorname{ring} \mathbb{Z}[G]$. In the case of homology cobordisms, the resulting functor would be equivalent to the (reduced) Magnus representation but it would miss the relative Reidemeister torsion: so it would be weaker than the functor $A$.

The paper is organized as follows. A first part deals exclusively with the Alexander functor: $\S 2$ gives the construction of the functor $A$ (Theorem I) and $\S 3$ explains how the classical Alexander polynomial of knots is contained in A. Next, the Reidemeister functor is constructed in $\S 4$ (Theorem II) and it is proved to be a generalization of $A$ in $\S 5$. (Thus, we provide two different proofs of the functoriality of A.) Starting from 
there, we focus on the study of $\mathrm{R}$ and indicate the resulting properties for $\mathrm{A}$. The abelian Reidemeister torsions of knot exteriors and closed 3-manifolds are shown to be determined by $\mathrm{R}$ in $\S 6$. The functor $\mathrm{R}$ restricts to a projective representation of the monoid of homology cobordisms, which we fully compute in $\S 7$. We also explain in $\S 8$ how to calculate R using Heegaard splittings of cobordisms, and we prove in $\S 9$ a duality result for $\mathrm{R}$ which involves the twisted intersection form of surfaces. Finally, the paper ends with a short appendix recalling the definition and basic properties of the torsion of chain complexes.

Notation and conventions. Let $R$ be a commutative ring. The exterior algebra of an $R$-module $N$ is denoted by

$$
\Lambda N=\bigoplus_{i \geq 0} \Lambda^{i} N \quad \text { where } \Lambda^{0} N=R
$$

the multivector $v_{1} \wedge \cdots \wedge v_{i} \in \Lambda^{i} N$ defined by a finite family $v=\left(v_{1}, \ldots, v_{i}\right)$ of elements of $N$ is still denoted by $v$. If $N$ is free of rank $n$, a volume form on $N$ is an isomorphism of $R$-modules $\Lambda^{n} N \rightarrow R$.

Let $X$ be a topological space with base point $\star$. The maximal abelian cover of $X$ based at $\star$ is denoted by $p_{X}: \widehat{X} \rightarrow X$, and the preferred lift of $\star$ is denoted by $\widehat{\star}$. (Here we assume the appropriate assumptions on $X$ to have a universal cover.) For any oriented loop $\alpha$ in $X$ based at $\star$, the unique lift of $\alpha$ to $\widehat{X}$ starting at $\widehat{\star}$ is denoted by $\widehat{\alpha}$.

Unless otherwise specified, (co)homology groups are taken with coefficients in the ring of integers $\mathbb{Z} ;($ co)homology classes are denoted with square brackets [-]. For any subspace $Y \subset X$ such that $\star \in Y$ and any ring homomorphism $\varphi: \mathbb{Z}\left[H_{1}(X)\right] \rightarrow R$, we denote by $H^{\varphi}(X, Y)$ the $\varphi$-twisted homology of the pair $(X, Y)$, namely

$$
H^{\varphi}(X, Y)=H\left(C^{\varphi}(X, Y)\right) \quad \text { where } C^{\varphi}(X, Y):=R \otimes_{\mathbb{Z}\left[H_{1}(X)\right]} C\left(\widehat{X}, p_{X}^{-1}(Y)\right) .
$$

If $\left(X^{\prime}, Y^{\prime}\right)$ is another pair of spaces and $f:\left(X^{\prime}, Y^{\prime}\right) \rightarrow(X, Y)$ is a continuous map, the corresponding homomorphism $H\left(X^{\prime}\right) \rightarrow H(X)$ is still denoted by $f$. If a base point $\star^{\prime} \in Y^{\prime}$ is given and $f\left(\star^{\prime}\right)=\star$, the $R$-linear map $H^{\varphi f}\left(X^{\prime}, Y^{\prime}\right) \rightarrow H^{\varphi}(X, Y)$ induced by $f$ is also denoted by $f$.

Acknowledgements. This work was partially supported by the French ANR research project "Interlow" (ANR-09-JCJC-0097-01). The authors would like to thank the referee for some useful comments.

\section{The Alexander functor A}

We firstly review the Alexander function of a 3-manifold with boundary following [Les98]. (Note that the terminology "Alexander function" has a very different meaning in [Tur86].) Next, we construct the Alexander functor A. In this section, we fix a finitely generated free abelian group $G$; the extension of a group homomorphism $\varphi: A \rightarrow G$ to a ring homomorphism $\mathbb{Z}[A] \rightarrow \mathbb{Z}[G]$ is still denoted by $\varphi$.

2.1. The Alexander function. Let $M$ be a compact connected orientable 3-manifold with connected boundary. We fix a base point $\star \in \partial M$ and a group homomorphism $\varphi: H_{1}(M) \rightarrow G$. The genus of $M$ is the integer $g(M):=1-\chi(M)$, i.e. the genus of the surface $\partial M$.

Lemma 2.1. There exists a presentation of the $\mathbb{Z}[G]$-module $H_{1}^{\varphi}(M, \star)$ whose deficiency is $g(M)$. 
Proof. We consider a decomposition of $M$ with a single 0-handle, $s$ 1-handles and $r 2$ handles. Since the boundary of $M$ has genus $g(M)$, we have $s-r=g(M)$. This handle decomposition defines a 2-dimensional complex $X \subset M$ onto which $M$ deformation retracts. The complex $X$ has a single 0-cell (which we assume to be $\star$ ), $s$ 1-cells and $r$ 2-cells. Thus we obtain a presentation of the $\mathbb{Z}[G]$-module $H_{1}^{\varphi}(M, \star) \simeq H_{1}^{\varphi}(X, \star)$ with $s$ generators and $r$ relations.

We now simplify our notation by setting $g:=g(M)$ and $H:=H_{1}^{\varphi}(M, \star)$.

Definition 2.2 (Lescop [Les98]). Consider a presentation of the $\mathbb{Z}[G]$-module $H$ with deficiency $g$ :

$$
H=\left\langle\gamma_{1}, \ldots, \gamma_{g+r} \mid \rho_{1}, \ldots, \rho_{r}\right\rangle .
$$

Let $\Gamma$ be the $\mathbb{Z}[G]$-module freely generated by the symbols $\gamma_{1}, \ldots, \gamma_{g+r}$, and regard $\rho_{1}, \ldots, \rho_{r}$ as elements of $\Gamma$. Then the Alexander function of $M$ with coefficients $\varphi$ is the $\mathbb{Z}[G]$-linear map $\mathcal{A}_{M}^{\varphi}: \Lambda^{g} H \rightarrow \mathbb{Z}[G]$ defined by

$$
\mathcal{A}_{M}^{\varphi}\left(u_{1} \wedge \cdots \wedge u_{g}\right) \cdot \gamma_{1} \wedge \cdots \wedge \gamma_{g+r}=\rho_{1} \wedge \cdots \wedge \rho_{r} \wedge \widetilde{u_{1}} \wedge \cdots \wedge \widetilde{u_{g}} \in \Lambda^{g+r} \Gamma
$$

for any $u_{1}, \ldots, u_{g} \in H$, which we lift to some $\widetilde{u_{1}}, \ldots, \widetilde{u_{g}} \in \Gamma$ in an arbitrary way.

The map $\mathcal{A}_{M}^{\varphi}$ can be concretely computed as follows: if one considers the $r \times(g+r)$ matrix defined by the presentation (2.1) of $H$, and if one adjoins to this matrix some row vectors giving $u_{1}, \ldots, u_{g}$ in the generators $\gamma_{1}, \ldots, \gamma_{g+r}$, then $\mathcal{A}_{M}^{\varphi}\left(u_{1} \wedge \cdots \wedge u_{g}\right)$ is the determinant of the resulting $(g+r) \times(g+r)$ matrix. It is shown in [Les98, $§ 3.1]$ that, up to multiplication by a unit of $\mathbb{Z}[G]$ (i.e., an element of $\pm G$ ), the map $\mathcal{A}_{M}^{\varphi}$ does not depend on the choice of the presentation (2.1).

Let $Q(G)$ be the field of fractions of $\mathbb{Z}[G]$. The following lemma, which is implicit in [Les98], shows that either the Alexander function is trivial or it induces by extension of scalars a volume form on $H_{Q}:=Q(G) \otimes_{\mathbb{Z}[G]} H$.

Lemma 2.3. We have $\operatorname{dim} H_{Q} \geq g$, and $\mathcal{A}_{M}^{\varphi} \neq 0$ if and only if $\operatorname{dim} H_{Q}=g$.

Proof. Let $A$ be the $r \times(g+r)$ matrix with entries in $\mathbb{Z}[G]$ corresponding to the presentation (2.1) of the $\mathbb{Z}[G]$-module $H$. The multiplication $v \mapsto v A$ defines a linear map $Q(G)^{r} \rightarrow Q(G)^{g+r}$ whose cokernel is $H_{Q}$. Therefore

$$
\operatorname{dim} H_{Q}=(g+r)-\operatorname{rank} A .
$$

Clearly, we have $\operatorname{rank} A \leq r$ so that $\operatorname{dim} H_{Q} \geq g$.

Assume that $\operatorname{dim} H_{Q}>g$ and let $A^{\prime}$ be a matrix obtained by adding $g$ arbitrary rows to $A$. Then rank $A<r$ so that all the minors of $A$ of order $r$ vanish. By expanding the determinant of $A^{\prime}$ successively along the last $g$ rows, we see that $\operatorname{det} A^{\prime}=0$ and deduce that $\mathcal{A}_{M}^{\varphi}=0$.

Assume that $\operatorname{dim} H_{Q}=g$. Then $\operatorname{rank} A=r$ so that $A$ has a non-zero minor $D$ of order $r$. Let $1 \leq i_{1}<\cdots<i_{g} \leq g+r$ be the indices of the columns of $A$ not pertaining to $D$. Then $\mathcal{A}_{M}^{\varphi}\left(\gamma_{i_{1}} \wedge \cdots \wedge \gamma_{i_{g}}\right)=D \neq 0$.

2.2. Definition of A. In order to define a functor A, we associate to any object $(g, \varphi)$ of $\mathrm{Cob}_{G}$ the exterior algebra

$$
\mathrm{A}(g, \varphi):=\Lambda H_{1}^{\varphi}\left(F_{g}, \star\right)
$$

of the $\mathbb{Z}[G]$-module $H^{\varphi}\left(F_{g}, \star\right)=H_{1}^{\varphi}\left(F_{g}, \star\right)$, which is free of rank $2 g$. Next, we associate to any morphism $(M, \varphi) \in \operatorname{Cob}_{G}\left(\left(g_{-}, \varphi_{-}\right),\left(g_{+}, \varphi_{+}\right)\right)$a $\mathbb{Z}[G]$-linear map

$$
\mathrm{A}(M, \varphi): \Lambda H_{1}^{\varphi_{-}}\left(F_{g_{-}}, \star\right) \longrightarrow \Lambda H_{1}^{\varphi_{+}}\left(F_{g_{+}}, \star\right)
$$


of degree $\delta g:=g_{+}-g_{-}$as follows. We denote by $I$ the interval $m(\star \times[-1,1])$, which connects the base point of the bottom surface $\partial_{-} M$ to that of the top surface $\partial_{+} M$. We set $H:=H_{1}^{\varphi}(M, I), H_{ \pm}:=H_{1}^{\varphi_{ \pm}}\left(F_{g_{ \pm}}, \star\right)$ and $g:=g_{+}+g_{-}$. Then, for any integer $j \geq 0$, the image $\mathrm{A}(M, \varphi)(x) \in \Lambda^{j+\delta g} H_{+}$of any $x \in \Lambda^{j} H_{-}$is defined by the following property:

$$
\forall y \in \Lambda^{g-j} H_{+}, \mathcal{A}_{M}^{\varphi}\left(\Lambda^{j} m_{-}(x) \wedge \Lambda^{g-j} m_{+}(y)\right)=\omega(\mathrm{A}(M, \varphi)(x) \wedge y) .
$$

Here $\omega: \Lambda^{2 g_{+}} H_{+} \rightarrow \mathbb{Z}[G]$ is an arbitrary volume form on $H_{+}$. Due to the choices of $\omega$ and of the presentation of $H$, the map $\mathrm{A}(M, \varphi)$ is only defined up to multiplication by an element of $\pm G$. Besides, observe that $A(M, \varphi)$ is trivial on $\Lambda^{j} H_{-}$for any $j<\max (0,-\delta g)$ and any $j>\min \left(g, 2 g_{-}\right)$.

The next two lemmas show that the above paragraph defines a monoidal functor $A$ from $\operatorname{Cob}_{G}$ to $\operatorname{grMod}_{\mathbb{Z}[G], \pm G}$, which proves Theorem I of the Introduction. The first lemma is related to Property 6 of the Alexander function in [Les98], while the second lemma seems to be new.

Lemma 2.4. For any morphisms $(M, \varphi) \in \operatorname{Cob}_{G}\left(\left(g_{-}, \varphi_{-}\right),\left(g_{+}, \varphi_{+}\right)\right)$and $(N, \psi) \in$ $\operatorname{Cob}_{G}\left(\left(h_{-}, \psi_{-}\right),\left(h_{+}, \psi_{+}\right)\right)$, we have

$$
\mathrm{A}((M, \varphi) \otimes(N, \psi))=\mathrm{A}(M, \varphi) \otimes \mathrm{A}(N, \psi) .
$$

Proof. We set $g:=g_{+}+g_{-}, h:=h_{+}+h_{-}, \delta g:=g_{+}-g_{-}, \delta h:=h_{+}-h_{-}$and

$$
\begin{gathered}
H_{ \pm}^{M}:=H_{1}^{\varphi_{ \pm}}\left(F_{g_{ \pm}}, \star\right), H_{ \pm}^{N}:=H_{1}^{\psi_{ \pm}}\left(F_{h_{ \pm}}, \star\right), H_{ \pm}:=H_{1}^{\varphi \pm \oplus} \psi_{ \pm}\left(F_{g_{ \pm}+h_{ \pm}}, \star\right), \\
H^{M}:=H_{1}^{\varphi}(M, I), \quad H^{N}:=H_{1}^{\psi}(N, I), \quad H:=H_{1}^{\varphi \oplus \psi}\left(M \sharp_{\partial} N, I\right) .
\end{gathered}
$$

In the statement of the lemma and in the proof below, we identify

$$
\mathrm{A}\left(\left(g_{ \pm}, \varphi_{ \pm}\right) \otimes\left(h_{ \pm}, \psi_{ \pm}\right)\right)=\mathrm{A}\left(g_{ \pm}+h_{ \pm}, \varphi_{ \pm} \oplus \psi_{ \pm}\right)=\Lambda H_{ \pm}=\Lambda\left(H_{ \pm}^{M} \oplus H_{ \pm}^{N}\right)
$$

in the obvious way with

$$
\Lambda H_{ \pm}^{M} \otimes \Lambda H_{ \pm}^{N}=\mathrm{A}\left(g_{ \pm}, \varphi_{ \pm}\right) \otimes \mathrm{A}\left(h_{ \pm}, \psi_{ \pm}\right)
$$

Since the intersection of $M$ and $N$ in $M \sharp_{\partial} N$ is a 2-disk which retracts onto $I$, the Mayer-Vietoris theorem gives an isomorphism $H^{M} \oplus H^{N} \stackrel{\simeq}{\longrightarrow} H$. If rank $H^{M}>g$, then $\mathcal{A}_{M}^{\varphi}=0$ by Lemma 2.3 so that $\mathrm{A}(M, \varphi)=0$; the same lemma applied to $N$ shows that

$$
\operatorname{rank} H=\operatorname{rank} H^{M}+\operatorname{rank} H^{N}>g+h
$$

so that $\mathrm{A}((M, \varphi) \otimes(N, \psi))=0$ and (2.2) trivially holds true. Therefore, we can assume in the sequel that $\operatorname{rank}\left(H^{M}\right)=g$ and $\operatorname{rank}\left(H^{N}\right)=h$.

Let $x:=x^{M} \otimes x^{N} \in \Lambda^{i} H_{-}^{M} \otimes \Lambda^{j} H_{-}^{N} \subset \Lambda^{i+j} H_{-}$: we aim at showing that $a:=$ $\mathrm{A}((M, \varphi) \otimes(N, \psi))(x)$ is equal to

$$
a^{\prime}:=(\mathrm{A}(M, \varphi) \otimes \mathrm{A}(N, \psi))(x)=(-1)^{i \delta h} \mathrm{~A}(M, \varphi)\left(x^{M}\right) \otimes \mathrm{A}(N, \psi)\left(x^{N}\right) .
$$

(Recall that we are using Koszul's rule in the definition of the tensor product of morphisms in the category $\operatorname{grMod}_{\mathbb{Z}[G], \pm G}$.) It is enough to prove that, for any integers $p, q \geq 0$ such that $p+q=(g+h)-(i+j)$ and any $y:=y^{M} \otimes y^{N} \in \Lambda^{p} H_{+}^{M} \otimes \Lambda^{q} H_{+}^{N} \subset \Lambda^{p+q} H_{+}$, the identity

$$
\omega(a \wedge y)=\omega\left(a^{\prime} \wedge y\right)
$$

holds true up to multiplication by an element of $\pm G$ independent of $x, y$ (and, in particular, independent of $i, j, p, q)$. In the sequel, we fix some volume forms $\omega^{M}$ and $\omega^{N}$ on 
$H_{+}^{M}$ and $H_{+}^{N}$ respectively, and we assume that the volume form $\omega$ on $H_{+}=H_{+}^{M} \oplus H_{+}^{N}$ is defined by

$$
\omega(u \wedge v)=\omega^{M}(u) \cdot \omega^{N}(v)
$$

for any $u \in \Lambda^{2 g_{+}} H_{+}^{M}$ and $v \in \Lambda^{2 h_{+}} H_{+}^{N}$. By definition of $\mathrm{A}$, we have

$$
\omega(a \wedge y)=\mathcal{A}_{M \sharp \partial N}^{\varphi \oplus \psi}\left(\Lambda^{i} m_{-}\left(x^{M}\right) \wedge \Lambda^{j} n_{-}\left(x^{N}\right) \wedge \Lambda^{p} m_{+}\left(y^{M}\right) \wedge \Lambda^{q} n_{+}\left(y^{N}\right)\right) .
$$

If $p>g-i$, then $i+p>\operatorname{rank}\left(H^{M}\right)$ by our assumptions, so that $\Lambda^{i} m_{-}\left(x^{M}\right) \wedge \Lambda^{p} m_{+}\left(y^{M}\right) \in$ $\Lambda^{i+p} H^{M}$ is torsion; we deduce that $\omega(a \wedge y)=0$; on the other hand, the degree of $\mathrm{A}(M, \varphi)\left(x^{M}\right) \wedge y^{M} \in \Lambda H_{+}^{M}$ is $i+\delta g+p>2 g_{+}$so that $\omega\left(a^{\prime} \wedge y\right)=0$ as well; thus (2.3) trivially holds true if $p>g-i$. If $p<g-i$, then $q>h-j$ and the same conclusion applies. Therefore, we can assume in the sequel that $p=g-i$ and $q=h-j$.

To proceed, we consider a presentation $H^{M}=\left\langle\gamma_{1}, \ldots, \gamma_{g+r} \mid \rho_{1}, \ldots, \rho_{r}\right\rangle$ and a presentation $H^{N}=\left\langle\mu_{1}, \ldots, \mu_{h+s} \mid \zeta_{1}, \ldots, \zeta_{s}\right\rangle$. By the above-mentioned isomorphism between $H^{M} \oplus H^{N}$ and $H$, we obtain a presentation

$$
H=\left\langle\gamma_{1}, \ldots, \gamma_{g+r}, \mu_{1}, \ldots, \mu_{h+s} \mid \rho_{1}, \ldots, \rho_{r}, \zeta_{1}, \ldots, \zeta_{s}\right\rangle .
$$

Note that, with these choices of presentations, the matrix corresponding to $H$ is the direct sum of the matrices corresponding to $H^{M}$ and $H^{N}$. Therefore, we get

$$
\begin{aligned}
\omega(a \wedge y) \stackrel{\stackrel{(2.5)}{=}}{=}(-1)^{i s+p(s+j)} \mathcal{A}_{M}^{\varphi}\left(\Lambda^{i} m_{-}\left(x^{M}\right) \wedge \Lambda^{g-i} m_{+}\left(y^{M}\right)\right) \\
\cdot \mathcal{A}_{N}^{\psi}\left(\Lambda^{j} n_{-}\left(x^{N}\right) \wedge \Lambda^{h-j} n_{+}\left(y^{N}\right)\right) \\
=(-1)^{i s+p(s+j)} \omega^{M}\left(\mathrm{~A}(M, \varphi)\left(x^{M}\right) \wedge y^{M}\right) \cdot \omega^{N}\left(\mathrm{~A}(N, \psi)\left(x^{N}\right) \wedge y^{N}\right) \\
\stackrel{(2.4)}{=}(-1)^{i s+p(s+j)} \omega\left(\mathrm{A}(M, \varphi)\left(x^{M}\right) \wedge y^{M} \wedge \mathrm{A}(N, \psi)\left(x^{N}\right) \wedge y^{N}\right) \\
=(-1)^{i s+p(s+j)+p(j+\delta h)} \omega\left(\mathrm{A}(M, \varphi)\left(x^{M}\right) \wedge \mathrm{A}(N, \psi)\left(x^{N}\right) \wedge y^{M} \wedge y^{N}\right) \\
=(-1)^{g(s+h)} \omega\left(a^{\prime} \wedge y\right) .
\end{aligned}
$$

Lemma 2.5. For any morphisms $(M, \varphi) \in \operatorname{Cob}_{G}\left(\left(g_{-}, \varphi_{-}\right),\left(g_{+}, \varphi_{+}\right)\right)$and $(N, \psi) \in$ $\operatorname{Cob}_{G}\left(\left(h_{-}, \psi_{-}\right),\left(h_{+}, \psi_{+}\right)\right)$such that $\left(g_{+}, \varphi_{+}\right)=\left(h_{-}, \psi_{-}\right)$, we have

$$
\mathrm{A}((N, \psi) \circ(M, \varphi))=\mathrm{A}(N, \psi) \circ \mathrm{A}(M, \varphi) .
$$

The next subsection is devoted to the proof of Lemma 2.5.

2.3. Proof of the functoriality of $A$. We use the notations of Lemma 2.5 and we set

$$
\begin{aligned}
& g:=g_{-}+g_{+}, \quad h:=h_{-}+h_{+}, \quad f:=g_{-}+h_{+}, \\
& \delta g:=g_{+}-g_{-}, \quad \delta h:=h_{+}-h_{-}, \quad \delta f:=h_{+}-g_{-}, \\
& H^{M}:=H_{1}^{\varphi}(M, I), \quad H^{N}:=H_{1}^{\psi}(N, I), \quad H:=H_{1}^{\psi+\varphi}(N \circ M, I) .
\end{aligned}
$$

Let $v=\left(v_{1}, \ldots, v_{2 g_{+}}\right)$be a basis of $H_{1}^{\varphi_{+}}\left(F_{g_{+}}, \star\right)$ : we set $m v_{i}:=m_{+}\left(v_{i}\right)$ and $n v_{i}:=n_{-}\left(v_{i}\right)$ for all $i=1, \ldots, 2 g_{+}$. We consider presentations of the following form:

$$
\begin{aligned}
H^{M} & =\left\langle m v_{1}, \ldots, m v_{2 g_{+}}, u_{1}, \ldots, u_{r} \mid \zeta_{1}, \ldots, \zeta_{r+\delta g}\right\rangle, \\
H^{N} & =\left\langle n v_{1}, \ldots, n v_{2 h_{-}}, w_{1}, \ldots, w_{s} \mid \rho_{1}, \ldots, \rho_{s-\delta h}\right\rangle .
\end{aligned}
$$

Applying the Mayer-Vietoris theorem to $N \circ M$, we obtain that the $\mathbb{Z}[G]$-module $H$ is generated by

$$
m v_{1}, \ldots, m v_{2 g_{+}}, n v_{1}, \ldots, n v_{2 h_{-}}, u_{1}, \ldots, u_{r}, w_{1}, \ldots, w_{s}
$$

subject to the relations $\zeta_{1}, \ldots, \zeta_{r+\delta g}, \rho_{1}, \ldots, \rho_{s-\delta h}, m v_{1}-n v_{1}, \ldots, m v_{2 g_{+}}-n v_{2 g_{+}}$. 
In the sequel, we set $H_{-}:=H_{1}^{\varphi_{-}}\left(F_{g_{-}}, \star\right)$ and $H_{+}:=H_{1}^{\psi_{+}}\left(F_{h_{+}}, \star\right)$. Let $x \in \Lambda^{j} H_{-}$and $y \in \Lambda^{f-j} H_{+}$: we wish to compute

$$
\mathcal{A}_{N \circ M}^{\psi+\varphi}\left(\Lambda^{j} m_{-}(x) \wedge \Lambda^{f-j} n_{+}(y)\right)
$$

using the previous presentation of $H$. For this, we do some computations in $\Lambda^{k} \Gamma$ where $k:=4 g_{+}+r+s$ and $\Gamma$ denotes the free $\mathbb{Z}[G]$-module generated by the $k$ symbols listed at (2.6). Set $\zeta:=\zeta_{1} \wedge \cdots \wedge \zeta_{r+\delta g}, \rho:=\rho_{1} \wedge \cdots \wedge \rho_{s-\delta h}$. Then, we have

$$
\begin{aligned}
& \zeta \wedge \rho \wedge\left(m v_{1}-n v_{1}\right) \wedge \cdots \wedge\left(m v_{2 g_{+}}-n v_{2 g_{+}}\right) \wedge \widehat{\Lambda^{j m_{-}}(x)} \wedge \widehat{\Lambda^{f-j_{n_{+}}}(y)} \\
& =\sum_{P}(-1)^{|P|} \varepsilon_{P} \cdot \zeta \wedge \rho \wedge m v_{P} \wedge n v_{\bar{P}} \wedge \widehat{\Lambda^{j} m_{-}(x)} \wedge \widehat{\Lambda^{f-j} n_{+}}(y) \\
& =\sum_{P}(-1)^{|P|(j+1)} \varepsilon_{P} \cdot\left(\zeta \wedge m v_{P} \wedge \widehat{\Lambda^{j} m_{-}(x)}\right) \wedge\left(\rho \wedge n v_{\bar{P}} \wedge \widehat{\Lambda^{f-j} n_{+}}(y)\right) \in \Lambda^{k} \Gamma .
\end{aligned}
$$

Here the sums are taken over all parts $P \subset\left\{1, \ldots, 2 g_{+}\right\}, \bar{P}$ denotes the complement of $P, m v_{P}$ is the wedge of the $m v_{i}$ for $i \in P, n v_{\bar{P}}$ is the wedge of the $n v_{i}$ for $i \in \bar{P}$ and $\varepsilon_{P}$ is the signature of the permutation $P \bar{P}$ (where the elements of $P$ in increasing order are followed by the elements of $\bar{P}$ in increasing order). A sign $(-1)^{(s-\delta h)(j+|P|)}$ is missing in the second sum but, since the presentation of $H^{N}$ is arbitrary of deficiency $h$, we can assume that its number of relations $(s-\delta h)$ is even.

In the sequel, we omit the "tilde" notation to distinguish elements of $\Lambda H$ from their lifts to $\Lambda \Gamma$. Note that, in the above sums, the multivector $\zeta \wedge m v_{P} \wedge \Lambda^{j} m_{-}(x)$ has degree $(r+\delta g)+|P|+j$ which is greater than $2 g_{+}+r$ as soon as $|P|>g-j$; similarly, the multivector $\rho \wedge n v_{\bar{P}} \wedge \Lambda^{f-j} n_{+}(y)$ has degree $(s-\delta h)+\left(2 g_{+}-|P|\right)+(f-j)$ which is greater than $2 h_{-}+s$ as soon as $|P|<g-j$; since $2 g_{+}+r$ and $2 h_{-}+s$ are respectively the numbers of generators of $H^{M}$ and $H^{N}$ in the above presentations, the summand corresponding to $P$ vanishes for $|P|>g-j$ and for $|P|<g-j$. Therefore the above sums are actually indexed by the subsets $P \subset\left\{1, \ldots, 2 g_{+}\right\}$having cardinality $g-j$, and we get

$$
\begin{aligned}
& \zeta \wedge \rho \wedge\left(m v_{1}-n v_{1}\right) \wedge \cdots \wedge\left(m v_{2 g_{+}}-n v_{2 g_{+}}\right) \wedge \Lambda^{j} m_{-}(x) \wedge \Lambda^{f-j} n_{+}(y) \\
= & \sum_{|P|=g-j} \varepsilon_{P}^{\prime} \cdot\left(\zeta \wedge m v_{P} \wedge \Lambda^{j} m_{-}(x)\right) \wedge\left(\rho \wedge n v_{\bar{P}} \wedge \Lambda^{f-j} n_{+}(y)\right)
\end{aligned}
$$

where we have set $\varepsilon_{P}^{\prime}:=(-1)^{|P|(j+1)} \varepsilon_{P}$. The summand is here equal to

$$
\begin{aligned}
& \varepsilon_{P}^{\prime} \cdot\left(\zeta \wedge m v_{P} \wedge \Lambda^{j} m_{-}(x)\right) \wedge\left(\rho \wedge n v_{\bar{P}} \wedge \Lambda^{f-j} n_{+}(y)\right) \\
= & \varepsilon_{P}^{\prime} \cdot\left(\mathcal{A}_{M}^{\varphi}\left(m v_{P} \wedge \Lambda^{j} m_{-}(x)\right) \cdot(m v \wedge u)\right) \\
& \wedge\left(\mathcal{A}_{N}^{\psi}\left(n v_{\bar{P}} \wedge \Lambda^{f-j} n_{+}(y)\right) \cdot(n v \wedge w)\right) \\
= & \varepsilon_{P}^{\prime} \cdot \mathcal{A}_{M}^{\varphi}\left(m v_{P} \wedge \Lambda^{j} m_{-}(x)\right) \mathcal{A}_{N}^{\psi}\left(n v_{\bar{P}} \wedge \Lambda^{f-j} n_{+}(y)\right) \cdot(m v \wedge n v \wedge u \wedge w) .
\end{aligned}
$$

We deduce that

$$
\begin{aligned}
& \mathcal{A}_{N \circ M}^{\psi+\varphi}\left(\Lambda^{j} m_{-}(x) \wedge \Lambda^{f-j} n_{+}(y)\right) \\
= & \sum_{|P|=g-j} \varepsilon_{P}^{\prime} \cdot \mathcal{A}_{M}^{\varphi}\left(m v_{P} \wedge \Lambda^{j} m_{-}(x)\right) \cdot \mathcal{A}_{N}^{\psi}\left(n v_{\bar{P}} \wedge \Lambda^{f-j} n_{+}(y)\right) \\
= & \mathcal{A}_{N}^{\psi}\left(\sum_{|P|=g-j} \varepsilon_{P}^{\prime} \cdot \mathcal{A}_{M}^{\varphi}\left(m v_{P} \wedge \Lambda^{j} m_{-}(x)\right) \cdot n v_{\bar{P}} \wedge \Lambda^{f-j} n_{+}(y)\right)
\end{aligned}
$$




$$
=\mathcal{A}_{N}^{\psi}\left(\sum_{|P|=g-j}(-1)^{|P|} \varepsilon_{P} \cdot \omega\left(\mathrm{A}(M, \varphi)(x) \wedge v_{P}\right) \cdot n v_{\bar{P}} \wedge \Lambda^{f-j} n_{+}(y)\right) .
$$

We can assume that the basis $v$ of $H_{1}^{\varphi_{+}}\left(F_{g_{+}}, \star\right)$ is compatible with the chosen volume form $\omega$, in the sense that $\omega\left(v_{1} \wedge \cdots \wedge v_{2 g_{+}}\right)=1$. Observe that, for all $z \in$ $\Lambda^{j+\delta g} H_{1}^{\varphi_{+}}\left(F_{g_{+}}, \star\right)$, we have the identities

$$
z=\sum_{|P|=g-j} \varepsilon_{\bar{P}} \cdot \omega\left(z \wedge v_{P}\right) \cdot v_{\bar{P}}=\sum_{|P|=g-j}(-1)^{|P|} \cdot \varepsilon_{P} \cdot \omega\left(z \wedge v_{P}\right) \cdot v_{\bar{P}}
$$

where the sums range over all subsets $P \subset\left\{1, \ldots, 2 g_{+}\right\}$of cardinality $g-j$. Hence

$$
\begin{aligned}
\mathcal{A}_{N \circ M}^{\psi+\varphi}\left(\Lambda^{j} m_{-}(x) \wedge \Lambda^{f-j} n_{+}(y)\right) & =\mathcal{A}_{N}^{\psi}\left(\Lambda^{j+\delta g} n_{-} \mathrm{A}(M, \varphi)(x) \wedge \Lambda^{f-j} n_{+}(y)\right) \\
& =\omega(\mathrm{A}(N, \psi)(\mathrm{A}(M, \varphi)(x)) \wedge y) .
\end{aligned}
$$

It follows that $\omega(\mathrm{A}((N, \psi) \circ(M, \varphi))(x) \wedge y)=\omega(\mathrm{A}(N, \psi)(\mathrm{A}(M, \varphi)(x)) \wedge y)$, which concludes the proof of Lemma 2.5.

\section{Alexander FunCtor And KnOts}

In this section, we relate the functor A to the classical Alexander polynomial of knots. We fix a finitely generated free abelian group $G$; the extension of a group homomorphism $\varphi: A \rightarrow G$ to a ring homomorphism $\mathbb{Z}[A] \rightarrow \mathbb{Z}[G]$ is still denoted by $\varphi$.

3.1. The Alexander polynomial of a topological pair. Given a finitely generated $\mathbb{Z}[G]$-module $N$ and an integer $i \geq 0$, the $i$-th Alexander polynomial of $N$ is the greatest common divisor of all minors of order $n-i$ in an $m \times n$ presentation matrix of $N$. This algebraic invariant is denoted by $\Delta_{i} N \in \mathbb{Z}[G] / \pm G$.

Let $(X, Y)$ be a pair of topological spaces, and assume that they have the homotopy type of finite $\mathrm{CW}$-complexes. Consider a group homomorphism $\varphi: H_{1}(X) \rightarrow G$. The Alexander polynomial of $(X, Y)$ with coefficients $\varphi$ is

$$
\Delta^{\varphi}(X, Y):=\Delta_{0} H_{1}^{\varphi}(X, Y) \in \mathbb{Z}[G] / \pm G .
$$

If $Y$ is empty, we set $\Delta^{\varphi}(X):=\Delta_{0} H_{1}^{\varphi}(X)$.

3.2. The Alexander function in genus one. Let $M$ be a compact connected orientable 3-manifold with connected boundary, and fix a base point $\star \in \partial M$. Let also $\varphi: H_{1}(M) \rightarrow G$ be a group homomorphism. The next lemma generalizes Property 1 of the Alexander function given in [Les98].

Lemma 3.1. Assume that $g(M)=1$ and that $\varphi$ is not trivial. Then, for any $h \in H:=$ $H_{1}^{\varphi}(M, \star)$, we have

$$
\mathcal{A}_{M}^{\varphi}(h)= \begin{cases}\Delta^{\varphi}(M) \cdot \partial_{*}(h) & \text { if } \operatorname{rank} \varphi\left(H_{1}(M)\right) \geq 2, \\ \Delta^{\varphi}(M) \cdot \frac{\partial_{*}(h)}{t-1} & \text { if } \operatorname{rank} \varphi\left(H_{1}(M)\right)=1 \text { and } t \text { is a generator. }\end{cases}
$$

Here $\partial_{*}: H \rightarrow \mathbb{Z}[G]$ is the connecting homomorphism $H_{1}^{\varphi}(M, \star) \rightarrow H_{0}^{\varphi}(\star)$ in the long exact sequence of the pair $(M, \star)$, followed by the canonical isomorphism $H_{0}^{\varphi}(\star) \simeq \mathbb{Z}[G]$.

We shall deduce Lemma 3.1 from the following.

Lemma 3.2. If $\varphi$ is not trivial, then $\Delta^{\varphi}(M)=\Delta_{1} H_{1}^{\varphi}(M, \star)$. 
Proof. The long exact sequence in $\varphi$-twisted homology for the pair $(M, \star)$ gives

$$
0 \longrightarrow H_{1}^{\varphi}(M) \longrightarrow H_{1}^{\varphi}(M, \star) \longrightarrow H_{0}^{\varphi}(\star) \longrightarrow H_{0}^{\varphi}(M) \longrightarrow 0 .
$$

Since the $\mathbb{Z}[G]$-module $H_{0}^{\varphi}(\star) \simeq \mathbb{Z}[G]$ is torsion-free, we deduce that

$$
\text { Tors } H_{1}^{\varphi}(M) \simeq \text { Tors } H_{1}^{\varphi}(M, \star) .
$$

Besides, the above exact sequence implies that

$$
\operatorname{rank} H_{1}^{\varphi}(M)-\operatorname{rank} H_{1}^{\varphi}(M, \star)+1-\operatorname{rank} H_{0}^{\varphi}(M)=0 .
$$

We now show that $\operatorname{rank} H_{0}^{\varphi}(M)=0$. By considering a cell decomposition of $M$ with $\star$ as a single 0 -cell and some 1-cells $e_{1}, \ldots, e_{r}$, we see that

$$
H_{0}^{\varphi}(M) \simeq \mathbb{Z}[G] /\left\langle\left(g_{1}-1\right), \ldots,\left(g_{r}-1\right)\right\rangle_{\text {ideal }}
$$

where $g_{i}:=\varphi\left(\left[e_{i}\right]\right) \in G$. Thus we have the short exact sequence of modules

$$
0 \longrightarrow I_{\varphi} \longrightarrow \mathbb{Z}[G] \longrightarrow H_{0}^{\varphi}(M) \longrightarrow 0,
$$

where $I_{\varphi}$ is the ideal generated by the $\varphi(h)-1$ for all $h \in H_{1}(M)$. By tensoring with the field of fractions $Q(G)$, we obtain

$$
0 \longrightarrow Q(G) \otimes_{\mathbb{Z}[G]} I_{\varphi} \longrightarrow Q(G) \longrightarrow Q(G) \otimes_{\mathbb{Z}[G]} H_{0}^{\varphi}(M) \longrightarrow 0
$$

Since $\varphi$ is not trivial, $Q(G) \otimes_{\mathbb{Z}[G]} I_{\varphi} \neq 0$ so that $Q(G) \otimes_{\mathbb{Z}[G]} H_{0}^{\varphi}(M)=0$. Hence

$$
\operatorname{rank} H_{1}^{\varphi}(M, \star)=\operatorname{rank} H_{1}^{\varphi}(M)+1 \text {. }
$$

We conclude thanks to (3.1) and (3.2) using the following:

Fact. [Bla57, Lemma 4.10]. Let $N$ be a finitely generated $\mathbb{Z}[G]$-module.

Then

$$
\Delta_{i}(N)= \begin{cases}0 & \text { if } i<\operatorname{rank}(N) \\ \Delta_{i-\operatorname{rank} N}(\operatorname{Tors} N) & \text { if } i \geq \operatorname{rank}(N) .\end{cases}
$$

Proof of Lemma 3.1. Observe that, for any oriented loop $\rho$ in $M$ based at $\star$, we have $\partial_{*}([\widehat{\rho}])=\varphi([\rho])-1$. Thus, the greatest common divisor of $\partial_{*}(H)$ is

$$
\operatorname{gcd} \partial_{*}(H)=\operatorname{gcd}\left\{\varphi(x)-1 \mid x \in H_{1}(M)\right\} \in \mathbb{Z}[G] / \pm G .
$$

Since $\varphi$ is assumed to be non-trivial, we deduce that

$$
\operatorname{gcd} \partial_{*}(H)= \begin{cases}1 & \text { if } \operatorname{rank} \varphi\left(H_{1}(M)\right) \geq 2, \\ t-1 & \text { if } \operatorname{rank} \varphi\left(H_{1}(M)\right)=1 \text { and } t \text { is a generator. }\end{cases}
$$

Therefore, we have to prove that

$$
\mathcal{A}_{M}^{\varphi}(h)=\Delta^{\varphi}(M) \cdot \frac{\partial_{*}(h)}{\operatorname{gcd} \partial_{*}(H)} .
$$

For this, we consider a presentation $H=\left\langle\gamma_{1}, \ldots, \gamma_{r+1} \mid \rho_{1}, \ldots, \rho_{r}\right\rangle$ and let $A$ be the associated $r \times(r+1)$ matrix. We have

$$
\forall z_{1}, \ldots, z_{r+1} \in \mathbb{Z}[G], \mathcal{A}_{M}^{\varphi}\left(z_{1} \gamma_{1}+\cdots+z_{r+1} \gamma_{r+1}\right)=\sum_{i=1}^{r+1}(-1)^{i+r+1} \operatorname{det}\left(A_{i}\right) z_{i}
$$

where $A_{i}$ is the matrix $A$ with the $i$-th column removed. Then Lemma 3.2 gives

$$
\Delta^{\varphi}(M)=\Delta_{1} H=\operatorname{gcd} \mathcal{A}_{M}^{\varphi}(H) \text {. }
$$

It follows that $\Delta^{\varphi}(M)=0$ if and only if $\mathcal{A}_{M}^{\varphi}=0$. In that case (3.3) trivially holds true: thus we assume in the sequel that $\mathcal{A}_{M}^{\varphi} \neq 0$. Lemma 2.3 implies that rank $H=1$ : it follows that any two $Q(G)$-linear maps $Q(G) \otimes_{\mathbb{Z}[G]} H \rightarrow Q(G)$ are linearly dependent. 
Since $\mathcal{A}_{M}^{\varphi} \neq 0$ and $\partial_{*} \neq 0$, we deduce that there exist non-zero elements $D, E \in \mathbb{Z}[G]$ such that

$$
\forall h \in H, \quad \mathcal{A}_{M}^{\varphi}(h)=\frac{D}{E} \cdot \partial_{*}(h)
$$

or, equivalently, $D \partial_{*}(h)=E \mathcal{A}_{M}^{\varphi}(h)$ for all $h \in H$. Hence $D \operatorname{gcd} \partial_{*}(H)=E \operatorname{gcd} \mathcal{A}_{M}^{\varphi}(H)$ and we deduce from (3.4) that

$$
\frac{D}{E}=\frac{\Delta^{\varphi}(M)}{\operatorname{gcd} \partial_{*}(H)}
$$

The identity (3.3) is then deduced from (3.5) and (3.6).

3.3. The functor $\mathrm{A}$ on knot exteriors. Let $K$ be an oriented knot in an oriented homology 3 -sphere $N$. The Alexander polynomial of $K$ is classically defined as

$$
\Delta(K):=\Delta^{\varphi_{K}}\left(M_{K}\right)=\Delta_{0} H_{1}^{\varphi_{K}}\left(M_{K}\right) \in \mathbb{Z}[G] / \pm G
$$

where $M_{K}$ is the complement of an open tubular neighborhood of $K$ in $N, G$ is the infinite cyclic group spanned by $t$, and $\varphi_{K}: H_{1}\left(M_{K}\right) \rightarrow G$ is the isomorphism mapping an oriented meridian $\mu \subset \partial M_{K}$ of $K$ to $t$. Note that $\Delta(K)$ is a Laurent polynomial in the variable $t$, which is defined up to multiplication by a monomial $\pm t^{k}$ for $k \in \mathbb{Z}$.

We make $M_{K}$ a morphism $1 \rightarrow 0$ in the category Cob by choosing a boundaryparametrization $m: F(1,0) \rightarrow \partial M_{K}$ such that $\mu_{-}:=m^{-1}(\mu)$ is contained in the bottom surface $F_{1}$ and goes through the base point $\star$. Set $H_{-}:=H_{1}^{\varphi_{K} m_{-}}\left(F_{1}, \star\right)$. The following proposition shows that the knot invariants $\Delta(K)$ and $\mathrm{A}\left(M_{K}, \varphi_{K}\right)$ carry the same topological information. This is deduced from Lemma 3.1 applied to $M:=M_{K}$.

Proposition 3.3. With the above notation and for any $h \in \Lambda^{i} H_{-}$, we have

$$
\mathrm{A}\left(M_{K}, \varphi_{K}\right)(h)= \begin{cases}\Delta(K) \cdot \partial_{*}(h) /(t-1) & \text { if } i=1, \\ 0 & \text { otherwise, }\end{cases}
$$

where $\partial_{*}: H_{-} \rightarrow \mathbb{Z}[G]$ is the connecting homomorphism for the pair $\left(F_{1}, \star\right)$. In particular, we have $\Delta(K)=\mathrm{A}\left(M_{K}, \varphi_{K}\right)\left(\left[\widehat{\mu}_{-}\right]\right)$.

\section{The ReIDEMEISTER FUnCTOR R}

In this section, we construct the Reidemeister functor $\mathrm{R}$. We fix a field $\mathbb{F}$ and a subgroup $G$ of $\mathbb{F}^{\times}$. In this section, the extension of a group homomorphism $\varphi: A \rightarrow G$ to a ring homomorphism $\mathbb{Z}[A] \rightarrow \mathbb{F}$ is still denoted by $\varphi$.

4.1. The Reidemeister function. We use the elementary theory of abelian Reidemeister torsions to construct an analogue of the Alexander function considered in $§ 2.1$. Let $M$ be a compact connected orientable 3-manifold with connected boundary, and let $\varphi: H_{1}(M) \rightarrow G$ be a group homomorphism. We fix a base point $\star \in \partial M$ and we set $g:=g(M)=1-\chi(M)$.

Lemma 4.1. We have $H_{i}^{\varphi}(M, \star)=0$ if $i=0$ or $i>2$. Moreover, we have

$$
\operatorname{dim} H_{1}^{\varphi}(M, \star)=g+\operatorname{dim} H_{2}^{\varphi}(M, \star) .
$$

Proof. Since $\partial M$ is non-empty, $M$ deformation retracts to a connected 2-dimensional complex whose only 0 -cell is $\star$ : the first assertion follows. Moreover, we have

$$
-g=\chi(M)-1=\chi(M, \star)=-\operatorname{dim} H_{1}^{\varphi}(M, \star)+\operatorname{dim} H_{2}^{\varphi}(M, \star) .
$$


Denote $H:=H_{1}^{\varphi}(M, \star)$ and assume in this paragraph that $\operatorname{dim} H=g$. We choose a cell decomposition of $M$ where $\star$ is a 0-cell: by Lemma 4.1, the homology of the $\varphi$-twisted cell chain complex $C^{\varphi}(M, \star)$ is concentrated in degree 1. For every dimension $i \in\{0, \ldots, 3\}$, let $n_{i} \geq 0$ be the number of relative $i$-cells of $(M, \star)$ and order them $\sigma_{1}^{(i)}, \ldots, \sigma_{n_{i}}^{(i)}$ in an arbitrary way. For every cell $\sigma$ of $(M, \star)$, we also choose an orientation of $\sigma$ and a lift $\hat{\sigma}$ of $\sigma$ to the maximal abelian cover $\widehat{M}$ of $M$. Thus, we get a basis $c:=\left(c_{3}, c_{2}, c_{1}, c_{0}\right)$ of the $\mathbb{F}$-chain complex $C^{\varphi}(M, \star)$ where, for every $i \in\{0, \ldots, 3\}$, the basis of the $\mathbb{F}$-vector space $C_{i}^{\varphi}(M, \star)$ is given by $c_{i}:=\left(1 \otimes \hat{\sigma}_{1}^{(i)}, \ldots, 1 \otimes \hat{\sigma}_{n_{i}}^{(i)}\right)$. Then we consider the function $H^{g} \rightarrow \mathbb{F}$ defined by

$$
\left(h_{1}, \ldots, h_{g}\right) \longmapsto \begin{cases}\tau\left(C^{\varphi}(M, \star) ; c,\left(h_{1}, \ldots, h_{g}\right)\right) & \text { if } h_{1} \wedge \cdots \wedge h_{g} \neq 0 \\ 0 & \text { otherwise }\end{cases}
$$

Here $\tau(C ; c, h)$ denotes the torsion of the finite $\mathbb{F}$-chain complex $C$ with basis $c$ and homological basis $h$ : see $\S$ A.1. It follows from the definition of the torsion that the map (4.1) is multilinear and alternate: see Lemma A.2.

Definition 4.2. The Reidemeister function of $M$ with coefficients $\varphi$ is the $\mathbb{F}$-linear map $\mathcal{R}_{M}^{\varphi}: \Lambda^{g} H \rightarrow \mathbb{F}$ defined by (4.1) if $\operatorname{dim} H=g$ and by $\mathcal{R}_{M}^{\varphi}:=0$ if $\operatorname{dim} H \neq g$.

Because of the choice of the orders, orientations, and lifts of the cells of $(M, \star)$, the map $\mathcal{R}_{M}^{\varphi}$ is only defined up to multiplication by an element of $\pm G \subset \mathbb{F}$. It remains to justify that $\mathcal{R}_{M}^{\varphi} \in \operatorname{Hom}\left(\Lambda^{g} H, \mathbb{F}\right) / \pm G$ defines a topological invariant of $M$ (i.e., it does not depend on the choice of the cell decomposition). Note that we do not need Chapman's result on the topological invariance of the torsion of CW-complexes [Cha74, Coh73] since we are considering here manifolds of dimension 3. Specifically, using Whitehead's theory of smooth triangulations and the fact that the Reidemeister torsion of CW-complexes is invariant under cellular subdivisions, we obtain that the above definition of $\mathcal{R}_{M}^{\varphi}$ applied to a smooth triangulation of $(M, \star)$ produces an invariant of smooth 3-manifolds. (See [Mil66, §9] or [Tur89, §3] for similar arguments which are valid in any dimension.) Next, we appeal to the 3-dimensional Hauptvermutung to conclude that $\mathcal{R}_{M}^{\varphi}$ is an invariant of topological 3-manifolds. Thus, we can consider in Definition 4.2 an arbitrary cell decomposition of $(M, \star)$ provided it can be subdivided to a smooth triangulation of $M$.

4.2. Definition of $\mathrm{R}$. The definition of the functor $\mathrm{R}$ from the Reidemeister function $\mathcal{R}$ goes parallel to the definition of $\mathrm{A}$ from $\mathcal{A}$ (see $\S 2.2$ ). Thus we associate to any object $(g, \varphi)$ of $\operatorname{Cob}_{G}$ the exterior algebra

$$
\mathrm{R}(g, \varphi):=\Lambda H_{1}^{\varphi}\left(F_{g}, \star\right)
$$

of the $\mathbb{F}$-vector space $H^{\varphi}\left(F_{g}, \star\right)=H_{1}^{\varphi}\left(F_{g}, \star\right)$, which has dimension $2 g$. Next, we associate to any morphism $(M, \varphi)$ from $\left(g_{-}, \varphi_{-}\right)$to $\left(g_{+}, \varphi_{+}\right)$an $\mathbb{F}$-linear map

$$
\mathrm{R}(M, \varphi): \Lambda H_{1}^{\varphi_{-}}\left(F_{g_{-}}, \star\right) \longrightarrow \Lambda H_{1}^{\varphi_{+}}\left(F_{g_{+}}, \star\right)
$$

of degree $\delta g:=g_{+}-g_{-}$in the following way. We set $H:=H_{1}^{\varphi}(M, I)$ where $I:=$ $m(\star \times[-1,1]), H_{ \pm}:=H_{1}^{\varphi_{ \pm}}\left(F_{g_{ \pm}}, \star\right)$ and $g:=g_{+}+g_{-}$. Then, for any integer $j \geq 0$, the image $\mathrm{R}(M, \varphi)(x) \in \Lambda^{j+\delta g} H_{+}$of any $x \in \Lambda^{j} H_{-}$is defined by the following property:

$$
\forall y \in \Lambda^{g-j} H_{+}, \mathcal{R}_{M}^{\varphi}\left(\Lambda^{j} m_{-}(x) \wedge \Lambda^{g-j} m_{+}(y)\right)=\omega(\mathrm{R}(M, \varphi)(x) \wedge y) .
$$

Here $\omega: \Lambda^{2 g_{+}} H_{+} \rightarrow \mathbb{F}$ is an arbitrary volume form which is integral in the following sense: regarding $H_{+}$as $\mathbb{F} \otimes_{\mathbb{Z}\left[H_{1}\left(F_{g_{+}}\right)\right]} H_{1}\left(F_{g_{+}}, \star ; \mathbb{Z}\left[H_{1}\left(F_{g_{+}}\right)\right]\right)$, we assume that $\omega$ arises from an arbitrary volume form on the free $\mathbb{Z}\left[H_{1}\left(F_{g_{+}}\right)\right]$-module $H_{1}\left(F_{g_{+}}, \star ; \mathbb{Z}\left[H_{1}\left(F_{g_{+}}\right)\right]\right)$. Due to the choices of this volume form and of the ordered/oriented lifts of the cells to 
$\widehat{M}$, the map $\mathrm{R}(M, \varphi)$ is only defined up to multiplication by an element of $\pm G \subset \mathbb{F}$. Besides, $R(M, \varphi)$ is trivial on $\Lambda^{j} H_{-}$for any $j<\max (0,-\delta g)$ and any $j>\min \left(g, 2 g_{-}\right)$.

The next two lemmas show that the above paragraph defines a monoidal functor $\mathrm{R}: \operatorname{Cob}_{G} \rightarrow \operatorname{grVect}_{\mathbb{F}, \pm G}$, which proves Theorem II of the Introduction.

Lemma 4.3. For any morphisms $(M, \varphi) \in \operatorname{Cob}_{G}\left(\left(g_{-}, \varphi_{-}\right),\left(g_{+}, \varphi_{+}\right)\right)$and $(N, \psi) \in$ $\operatorname{Cob}_{G}\left(\left(h_{-}, \psi_{-}\right),\left(h_{+}, \psi_{+}\right)\right)$, we have

$$
\mathrm{R}((M, \varphi) \otimes(N, \psi))=\mathrm{R}(M, \varphi) \otimes \mathrm{R}(N, \psi) .
$$

Proof. We set $g:=g_{+}+g_{-}, h:=h_{+}+h_{-}, \delta g:=g_{+}-g_{-}, \delta h:=h_{+}-h_{-}$and

$$
\begin{gathered}
H_{ \pm}^{M}:=H_{1}^{\varphi_{ \pm}}\left(F_{g_{ \pm}}, \star\right), H_{ \pm}^{N}:=H_{1}^{\psi_{ \pm}}\left(F_{h_{ \pm}}, \star\right), H_{ \pm}:=H_{1}^{\varphi_{ \pm} \oplus \psi_{ \pm}}\left(F_{g_{ \pm}+h_{ \pm}}, \star\right), \\
H^{M}:=H_{1}^{\varphi}(M, I), \quad H^{N}:=H_{1}^{\psi}(N, I), \quad H:=H_{1}^{\varphi \oplus \psi}\left(M_{\sharp_{\partial}} N, I\right) .
\end{gathered}
$$

Since $M$ and $N$ intersect in $M \sharp_{\partial} N$ along a 2-disk which retracts onto $I$, the MayerVietoris theorem gives an isomorphism $H^{M} \oplus H^{N} \stackrel{\simeq}{\longrightarrow} H$. If $\operatorname{dim}\left(H^{M}\right)>g$, then $\mathcal{R}_{M}^{\varphi}=0$ by definition, so that $\mathrm{R}(M, \varphi)=0$; moreover,

$$
\operatorname{dim}(H)=\operatorname{dim}\left(H^{M}\right)+\operatorname{dim}\left(H^{N}\right)>g+h
$$

so that $\mathrm{R}((M, \varphi) \otimes(N, \psi))=0$ as well, and (4.2) trivially holds true in that case. Therefore, we can assume that $\operatorname{dim}\left(H^{M}\right)=g$ and $\operatorname{dim}\left(H^{N}\right)=h$.

Let $x^{M}=\left(x_{1}^{M}, \ldots, x_{i}^{M}\right)$ be a family of vectors in $H_{-}^{M}$ and let $x^{N}=\left(x_{1}^{N}, \ldots, x_{j}^{N}\right)$ be a family of vectors in $H_{-}^{N}$. We consider the element

$$
x:=x^{M} \otimes x^{N} \in \Lambda^{i} H_{-}^{M} \otimes \Lambda^{j} H_{-}^{N} \subset \Lambda^{i+j}\left(H_{-}^{M} \oplus H_{-}^{N}\right)=\Lambda^{i+j} H_{-} .
$$

We aim at showing that $r:=\mathrm{R}((M, \varphi) \otimes(N, \psi))(x)$ is equal to

$$
r^{\prime}:=(\mathrm{R}(M, \varphi) \otimes \mathrm{R}(N, \psi))(x)=(-1)^{i \delta h} \cdot \mathrm{R}(M, \varphi)\left(x^{M}\right) \otimes \mathrm{R}(N, \psi)\left(x^{N}\right) .
$$

It is enough to prove that, for any integers $p, q \geq 0$ such that $p+q=(g+h)-(i+j)$ and for any families $y^{M}=\left(y_{1}^{M}, \ldots, y_{p}^{M}\right) \subset H_{+}^{M}$ and $y^{N}=\left(y_{1}^{N}, \ldots, y_{q}^{N}\right) \subset H_{+}^{N}$, we have

$$
\omega(r \wedge y)=\omega\left(r^{\prime} \wedge y\right)
$$

where $y:=y^{M} \otimes y^{N} \in \Lambda^{p} H_{+}^{M} \otimes \Lambda^{q} H_{+}^{N} \subset \Lambda^{p+q} H_{+}$. In fact, we only need to prove (4.3) up to multiplication by an element of $\pm G$, provided this factor is independent of $i, j, p, q, x$ and $y$.

In the sequel, we fix integral volume forms $\omega^{M}$ and $\omega^{N}$ on $H_{+}^{M}$ and $H_{+}^{N}$ respectively, and we assume that the volume form $\omega$ on $H_{+}=H_{+}^{M} \oplus H_{+}^{N}$ is defined by

$$
\omega(u \wedge v)=\omega^{M}(u) \cdot \omega^{N}(v)
$$

for any $u \in \Lambda^{2 g_{+}} H_{+}^{M}, v \in \Lambda^{2 h_{+}} H_{+}^{N}$. (So $\omega$ is integral too.) By definition of $\mathrm{R}$, we have

$$
\omega(r \wedge y)=\mathcal{R}_{M \sharp \partial N}^{\varphi \oplus \psi}\left(\Lambda^{i} m_{-}\left(x^{M}\right) \wedge \Lambda^{j} n_{-}\left(x^{N}\right) \wedge \Lambda^{p} m_{+}\left(y^{M}\right) \wedge \Lambda^{q} n_{+}\left(y^{N}\right)\right) .
$$

If $p>g-i$, then we have $i+p>\operatorname{dim}\left(H^{M}\right)$ by our assumptions and we obtain $\Lambda^{i} m_{-}\left(x^{M}\right) \wedge \Lambda^{p} m_{+}\left(y^{M}\right)=0 \in \Lambda^{i+p} H^{M}$; we deduce that $\omega(r \wedge y)=0$; on the other hand, the degree of the multivector $\mathrm{R}(M, \varphi)\left(x^{M}\right) \wedge y^{M} \in \Lambda H_{+}^{M}$ is $i+\delta g+p>2 g_{+}$so that $\omega\left(r^{\prime} \wedge y\right)=0$ as well; thus (4.3) trivially holds true if $p>g-i$. If $p<g-i$, then $q>h-j$ and the same conclusion applies. Therefore, we can assume that $p=g-i$ and $q=h-j$ in the sequel.

Since $H^{M} \oplus H^{N} \simeq H, k:=\left(m_{-}\left(x^{M}\right), m_{+}\left(y^{M}\right), n_{-}\left(x^{N}\right), n_{+}\left(y^{N}\right)\right)$ is a basis of $H$ if, and only if, the families $k^{M}:=\left(m_{-}\left(x^{M}\right), m_{+}\left(y^{M}\right)\right)$ and $k^{N}:=\left(n_{-}\left(x^{N}\right), n_{+}\left(y^{N}\right)\right)$ are 
basis of $H^{M}$ and $H^{N}$ respectively. If the former condition is not satisfied, then $\omega(r \wedge y)$ is zero by (4.5) and, if the latter condition is not satisfied, then $\omega\left(r^{\prime} \wedge y\right)$ is trivial as well since we have

$$
\begin{aligned}
\omega\left(r^{\prime} \wedge y\right) & =(-1)^{i \delta h} \omega\left(\mathrm{R}(M, \varphi)\left(x^{M}\right) \wedge \mathrm{R}(N, \psi)\left(x^{N}\right) \wedge y^{M} \wedge y^{N}\right) \\
= & (-1)^{i \delta h+p(j+\delta h)} \omega\left(\mathrm{R}(M, \varphi)\left(x^{M}\right) \wedge y^{M} \wedge \mathrm{R}(N, \psi)\left(x^{N}\right) \wedge y^{N}\right) \\
\stackrel{(4.4)}{=} & (-1)^{g h+p j} \omega^{M}\left(\mathrm{R}(M, \varphi)\left(x^{M}\right) \wedge y^{M}\right) \cdot \omega^{N}\left(\mathrm{R}(N, \psi)\left(x^{N}\right) \wedge y^{N}\right)
\end{aligned}
$$

or, equivalently,

$$
\begin{array}{r}
\omega\left(r^{\prime} \wedge y\right)=(-1)^{g h+p j} \mathcal{R}_{M}^{\varphi}\left(\Lambda^{i} m_{-}\left(x^{M}\right) \wedge \Lambda^{g-i} m_{+}\left(y^{M}\right)\right) \\
\cdot \mathcal{R}_{N}^{\psi}\left(\Lambda^{j} n_{-}\left(x^{N}\right) \wedge \Lambda^{h-j} n_{+}\left(y^{N}\right)\right) .
\end{array}
$$

Therefore, we can assume in the sequel that $k$ is a basis of $H$.

Consider next the twisted cell chain complexes $C:=C^{\varphi \oplus \psi}\left(M \sharp_{\partial} N, I\right), C^{M}:=C^{\varphi}(M, I)$ and $C^{N}:=C^{\psi}(N, I)$. There is a short exact sequence of $\mathbb{F}$-chain complexes

$$
0 \longrightarrow D \longrightarrow C^{M} \oplus C^{N} \longrightarrow C \longrightarrow 0
$$

where $D$ is the (un-)twisted cell chain complex of the disk $M \cap N \subset M \sharp_{\partial} N$ relatively to $I$. Clearly, $D$ is acyclic. By the multiplicativity property of torsions (see Theorem A.3 and Example A.4), we obtain

$$
\varepsilon \cdot \tau(C ; c, k) \cdot \tau(D ; d) \cdot \tau\left(\mathcal{H} ;\left(\left(k^{M}, k^{N}\right), k\right)\right)=\tau\left(C^{M} ; c^{M}, k^{M}\right) \cdot \tau\left(C^{N} ; c^{N}, k^{N}\right)
$$

for some appropriate choices of ordered/oriented lifts of the relative cells, which result in bases $c, d, c^{M}, c^{N}$ of the chain complexes. Here $\varepsilon$ is a sign not depending on $i, j, p, q, x, y$, and $\mathcal{H}$ is the long exact sequence in homology

$$
0 \longrightarrow \cdots \longrightarrow 0 \longrightarrow H^{M} \oplus H^{N} \longrightarrow H \longrightarrow 0 \longrightarrow 0 \longrightarrow 0
$$

induced by (4.7), which we view as a finite acyclic $\mathbb{F}$-chain complex concentrated in degrees 3,4 and with basis $\left(\left(k^{M}, k^{N}\right), k\right)$. By definition of $k, k^{M}$ and $k^{N}$, we have $\tau\left(\mathcal{H} ;\left(\left(k^{M}, k^{N}\right), k\right)\right)=1$ and, since the intersection disk $M \cap N$ can be reduced to $I$ by elementary collapses, the scalar $T:=\tau(D ; d)$ belongs to $\pm G$. We conclude that

$$
\begin{aligned}
& \omega(r \wedge y) \stackrel{(4.5)}{=}(-1)^{p j} \cdot \tau(C ; c, k) \\
& =(-1)^{p j} \varepsilon T^{-1} \cdot \tau\left(C^{M} ; c^{M}, k^{M}\right) \cdot \tau\left(C^{N} ; c^{N}, k^{N}\right) \\
& \stackrel{(4.6)}{=}(-1)^{g h} \varepsilon T^{-1} \cdot \omega\left(r^{\prime} \wedge y\right) \text {. }
\end{aligned}
$$

Lemma 4.4. For any morphisms $(M, \varphi) \in \operatorname{Cob}_{G}\left(\left(g_{-}, \varphi_{-}\right),\left(g_{+}, \varphi_{+}\right)\right)$and $(N, \psi) \in$ $\operatorname{Cob}_{G}\left(\left(h_{-}, \psi_{-}\right),\left(h_{+}, \psi_{+}\right)\right)$such that $\left(g_{+}, \varphi_{+}\right)=\left(h_{-}, \psi_{-}\right)$, we have

$$
\mathrm{R}((N, \psi) \circ(M, \varphi))=\mathrm{R}(N, \psi) \circ \mathrm{R}(M, \varphi) .
$$

The next subsection is devoted to the proof of Lemma 4.4 .

4.3. Proof of the functoriality of R. We use the notations of Lemma 4.4 and we set

$$
\begin{aligned}
g:=g_{-}+g_{+}, \quad h:=h_{-}+h_{+}, \quad f:=g_{-}+h_{+}, \\
\delta g:=g_{+}-g_{-}, \quad \delta h:=h_{+}-h_{-}, \quad \delta f:=h_{+}-g_{-}, \\
H^{M}:=H_{1}^{\varphi}(M, I), \quad H^{N}:=H_{1}^{\psi}(N, I), \quad H:=H_{1}^{\psi+\varphi}(N \circ M, I), \\
K^{M}:=H_{2}^{\varphi}(M, I), \quad K^{N}:=H_{2}^{\psi}(N, I), \quad K:=H_{2}^{\psi+\varphi}(N \circ M, I), \\
H_{-}:=H_{1}^{\varphi}\left(F_{g_{-}}, \star\right), \quad V:=H_{1}^{\varphi+}\left(F_{g_{+}}, \star\right), \quad H_{+}:=H_{1}^{\psi+}\left(F_{h_{+}}, \star\right) .
\end{aligned}
$$


Since $N \circ M$ is obtained from $M$ and $N$ by identifying $\partial_{+} M$ to $\partial_{-} N$, there is a short exact sequence of chain complexes

$$
0 \longrightarrow \underbrace{C^{\varphi_{+}}\left(F_{g_{+}}, \star\right)}_{D:=} \longrightarrow \underbrace{C^{\psi}(N, I)}_{C^{N}:=} \oplus \underbrace{C^{\varphi}(M, I)}_{C^{M}:=} \longrightarrow \underbrace{C^{\psi+\varphi}(N \circ M, I)}_{C:=} \longrightarrow 0 .
$$

Let $\mathcal{H}$ be the corresponding long exact sequence in homology:

$$
0 \rightarrow \cdots \rightarrow 0 \rightarrow K^{N} \oplus K^{M} \rightarrow K \rightarrow V \stackrel{\left(-n_{-}, m_{+}\right)}{\longrightarrow} H^{N} \oplus H^{M} \rightarrow H \rightarrow 0 \rightarrow 0 \rightarrow 0 .
$$

If $K^{M} \neq 0$, then $\operatorname{dim}\left(H^{M}\right)>g$ by Lemma 4.1 so that $\mathcal{R}_{M}^{\varphi}=0$ and $\mathrm{R}(M, \varphi)=0$; besides, the long exact sequence $\mathcal{H}$ implies that $K \neq 0$ so that $\mathrm{R}((N, \psi) \circ(M, \varphi))=0$; therefore, (4.8) trivially holds true in that case. If $K^{N} \neq 0$, the same conclusion applies. So, we can assume that $K^{M}=0$ and $K^{N}=0$ or, equivalently, $\operatorname{dim} H^{M}=g$ and $\operatorname{dim} H^{N}=h$.

Let $j \in\{0, \ldots, f\}$, and let $x=\left(x_{1}, \ldots, x_{j}\right)$ and $y=\left(y_{1}, \ldots, y_{f-j}\right)$ be families of vectors in $H_{-}$and $H_{+}$respectively. Let $v=\left(v_{1}, \ldots, v_{2 g_{+}}\right)$be an arbitrary basis of $V$ and let $\omega^{v}: \Lambda^{2 g_{+}} V \rightarrow \mathbb{F}$ be the volume form such that $\omega^{v}\left(v_{1} \wedge \cdots \wedge v_{2 g_{+}}\right)=1$; there exists an $\alpha_{v} \in \mathbb{F} \backslash\{0\}$ such that $\omega=\alpha_{v} \cdot \omega^{v}$ is the integral volume form chosen in the definition of the functor $\mathrm{R}$. We have $\mathrm{R}(M, \varphi)(x) \in \Lambda^{j+\delta g} V$, hence

$$
\mathrm{R}(M, \varphi)(x)=\sum_{|P|=g-j} \varepsilon_{\bar{P}} \cdot \omega^{v}\left(\mathrm{R}(M, \varphi)(x) \wedge v_{P}\right) \cdot v_{\bar{P}}
$$

where the sum is taken over all subsets $P \subset\left\{1, \ldots, 2 g_{+}\right\}$of cardinality $g-j, \bar{P}$ denotes the complement of $P, v_{P}$ (respectively $v_{\bar{P}}$ ) is the wedge of the $v_{i}$ 's for $i \in P$ (respectively $i \in \bar{P}$ ), and $\varepsilon_{\bar{P}}$ is the signature of the permutation $\bar{P} P$ (where the elements of $\bar{P}$ in increasing order are followed by the elements of $P$ in increasing order). We deduce that

$$
\omega(\mathrm{R}(N, \psi)(\mathrm{R}(M, \varphi)(x)) \wedge y)
$$

$$
\begin{aligned}
& =\mathcal{R}_{N}^{\psi}\left(\Lambda^{j+\delta g} n_{-} \mathrm{R}(M, \varphi)(x) \wedge \Lambda^{f-j} n_{+}(y)\right) \\
& =\mathcal{R}_{N}^{\psi}\left(\sum_{|P|=g-j} \varepsilon_{\bar{P}} \cdot \omega^{v}\left(\mathrm{R}(M, \varphi)(x) \wedge v_{P}\right) \cdot \Lambda^{j+\delta g_{-}} n_{-}\left(v_{\bar{P}}\right) \wedge \Lambda^{f-j} n_{+}(y)\right) \\
& =\alpha_{v}^{-1} \mathcal{R}_{N}^{\psi}\left(\sum_{|P|=g-j} \varepsilon_{P}^{\prime} \cdot \mathcal{R}_{M}^{\varphi}\left(\Lambda^{g-j} m_{+}\left(v_{P}\right) \wedge \Lambda^{j} m_{-}(x)\right) \cdot \Lambda^{j+\delta g_{-}} n_{-}\left(v_{\bar{P}}\right) \wedge \Lambda^{f-j} n_{+}(y)\right) \\
& =\alpha_{v}^{-1} \sum_{|P|=g-j} \varepsilon_{P}^{\prime} \cdot \mathcal{R}_{M}^{\varphi}\left(\Lambda^{g-j} m_{+}\left(v_{P}\right) \wedge \Lambda^{j} m_{-}(x)\right) \cdot \mathcal{R}_{N}^{\psi}\left(\Lambda^{j+\delta g} n_{-}\left(v_{\bar{P}}\right) \wedge \Lambda^{f-j} n_{+}(y)\right)
\end{aligned}
$$

where $\varepsilon_{P}^{\prime}:=\varepsilon_{\bar{P}} \cdot(-1)^{j(g-j)}$. If $K \neq 0$, then $\mathrm{R}((N, \psi) \circ(M, \varphi))=0$; besides, the long exact sequence in homology $\mathcal{H}$ shows that there exists a $w \in V \backslash\{0\}$ such that $n_{-}(w)=0 \in H^{N}$ and $m_{+}(w)=0 \in H^{M}$; since the basis $v$ of $V$ is arbitrary in (4.10), we can assume that $v_{1}=w$. In the last sum indexed by $P$, the vector $w$ appears either in $v_{P}$ or in $v_{\bar{P}}$, so that the corresponding summand is always zero; it follows that $\mathrm{R}(N, \psi)(\mathrm{R}(M, \varphi)(x)) \wedge y=0$ for any $x \in \Lambda^{j} H_{-}$and $y \in \Lambda^{f-j} H_{+}$; therefore, (4.8) trivially holds true in that case. Thus, we can assume in the sequel that $K=0$ or, equivalently, $\operatorname{dim} H=f$.

It now remains to prove using the above assumptions that, for any families of vectors $x=\left(x_{1}, \ldots, x_{j}\right)$ in $H_{-}$and $y=\left(y_{1}, \ldots, y_{f-j}\right)$ in $H_{+}$,

$$
\begin{aligned}
\text { (4.11) } & \omega(\mathrm{R}((N, \psi) \circ(M, \varphi))(x) \wedge y) \\
= & \alpha_{v}^{-1} \sum_{|P|=g-j} \varepsilon_{P}^{\prime} \cdot \mathcal{R}_{M}^{\varphi}\left(\Lambda^{g-j} m_{+}\left(v_{P}\right) \wedge \Lambda^{j} m_{-}(x)\right) \cdot \mathcal{R}_{N}^{\psi}\left(\Lambda^{j+\delta g_{n}} n_{-}\left(v_{\bar{P}}\right) \wedge \Lambda^{f-j} n_{+}(y)\right)
\end{aligned}
$$


where, as in the previous paragraph, $v$ is an arbitrary basis of $V$. Assume firstly that $k:=\left(m_{-}(x), n_{+}(y)\right)$ is not a basis of $H$. Then

$$
\mathrm{R}((N, \psi) \circ(M, \varphi))(x) \wedge y=\mathcal{R}_{N \circ M}^{\psi+\varphi}\left(\Lambda^{j} m_{-}(x) \wedge \Lambda^{f-j} n_{+}(y)\right)
$$

is zero. Besides, the long exact sequence $\mathcal{H}$ implies that there exists $w \in V$ such that

$$
\begin{aligned}
m_{+}(w) & =a_{1} m_{-}\left(x_{1}\right)+\cdots+a_{j} m_{-}\left(x_{j}\right) \in H^{M}, \\
-n_{-}(w) & =b_{1} n_{+}\left(y_{1}\right)+\cdots+b_{f-j} n_{+}\left(y_{f-j}\right) \in H^{N}
\end{aligned}
$$

where $a_{1}, \ldots, a_{j}, b_{1}, \ldots, b_{f-j} \in \mathbb{F}$ are not all zeroes. If $w=0$, then we have $\Lambda^{j} m_{-}(x)=$ $0 \in \Lambda^{j} H^{M}$ or $\Lambda^{f-j} n_{+}(y)=0 \in \Lambda^{f-j} H^{N}$ (depending on whether we can find a non-zero scalar among the $a_{i}$ 's or among the $b_{i}$ 's); in both cases, the second term of (4.11) is trivial. If $w \neq 0$, then we take a basis $v$ of $V$ such that $v_{1}=w$ and we easily see that the second term of (4.11) is trivial in that case too. Therefore, we can assume in the sequel that $k=\left(m_{-}(x), n_{+}(y)\right)$ is a basis of $H$.

We now fix a basis $v=\left(v_{1}, \ldots, v_{2 g_{+}}\right)$of $V$ such that $\omega(v)=1$ and we prove (4.11) with $\alpha_{v}=1$. Let also $k^{M}$ and $k^{N}$ be arbitrary bases of $H^{M}$ and $H^{N}$, respectively. By the multiplicativity property of torsions (see Theorem A.3 and Example A.4), we deduce from (4.9) that

$$
\begin{aligned}
& \tau(D ; d, v) \cdot \tau(C ; c, k) \cdot \tau\left(\mathcal{H} ;\left(v,\left(k^{N}, k^{M}\right), k\right)\right) \\
= & \pm \tau\left(C^{N} ; c^{N}, k^{N}\right) \cdot \tau\left(C^{M} ; c^{M}, k^{M}\right) \in \mathbb{F}
\end{aligned}
$$

for some appropriate choices of ordered/oriented lifts of the relative cells, which result in bases $d, c, c^{M}, c^{N}$ of the chain complexes. The sign appearing in (4.12) only depends on the dimensions of the complexes $C, D, C^{M}, C^{N}$ and the dimensions of their homology groups. The sequence $\mathcal{H}$ is viewed here as a finite acyclic $\mathbb{F}$-chain complex concentrated in degrees $3,4,5$; its torsion is

$$
\begin{aligned}
\tau\left(\mathcal{H} ;\left(v,\left(k^{N}, k^{M}\right), k\right)\right) & =\left[\frac{\left(\left(-n_{-}, m_{+}\right)(v), \text { lift of } k \text { to } H^{N} \oplus H^{M}\right)}{\left(k^{N}, k^{M}\right)}\right]^{-1} \\
& =\left[\frac{\left(k^{N}, k^{M}\right)}{\left(\left(-n_{-}, m_{+}\right)(v), \text { lift of } k \text { to } H^{N} \oplus H^{M}\right)}\right]
\end{aligned}
$$

where the symbol $\left[\frac{a}{b}\right]$ stands for the determinant of the square matrix expressing a family of vectors $a$ in the basis $b$ of $H^{N} \oplus H^{M}$. We have $\tau(D ; d, v) \in \pm G$ since $\left(F_{g_{+}}, \star\right)$ has the simple homotopy type of a wedge of circles relative to its vertex. We deduce from (4.12) that

$$
\begin{aligned}
& \mathcal{R}_{N \circ M}^{\psi+\varphi}\left(\Lambda^{j} m_{-}(x) \wedge \Lambda^{f-j} n_{+}(y)\right) \cdot\left[\frac{\left(k^{N}, k^{M}\right)}{\left(\left(-n_{-}, m_{+}\right)(v), \text { lift of } k \text { to } H^{N} \oplus H^{M}\right)}\right] \\
= & \beta_{v} \cdot \mathcal{R}_{M}^{\varphi}\left(k^{M}\right) \cdot \mathcal{R}_{N}^{\psi}\left(k^{N}\right)
\end{aligned}
$$

where $\beta_{v} \in \pm G$ does not depend on $j, x, y, k^{M}, k^{N}$ (but depends on $v$ ). The previous identity makes sense, and holds true, when $k^{M}$ is an arbitrary family of $g$ vectors in $H^{M}$ and $k^{N}$ is an arbitrary family of $h$ vectors in $H^{N}$. (Indeed, if $k^{M}$ is not a basis of $H^{M}$ or $k^{N}$ is not a basis of $H^{N}$, then both sides of this identity are zero.) In particular, we obtain for any subset $P \subset\left\{1, \ldots, 2 g_{+}\right\}$of cardinality $g-j$

$$
\mathcal{R}_{N \circ M}^{\psi+\varphi}\left(\Lambda^{j} m_{-}(x) \wedge \Lambda^{f-j} n_{+}(y)\right) \cdot\left[\frac{\left(n_{-}\left(v_{\bar{P}}\right), n_{+}(y), m_{+}\left(v_{P}\right), m_{-}(x)\right)}{\left(\left(-n_{-}, m_{+}\right)(v), \text { lift of } k \text { to } H^{N} \oplus H^{M}\right)}\right]
$$




$$
=\beta_{v} \cdot \mathcal{R}_{M}^{\varphi}\left(\Lambda^{g-j} m_{+}\left(v_{P}\right) \wedge \Lambda^{j} m_{-}(x)\right) \cdot \mathcal{R}_{N}^{\psi}\left(\Lambda^{\delta g+j} n_{-}\left(v_{\bar{P}}\right) \wedge \Lambda^{f-j} n_{+}(y)\right) .
$$

By multilinearity of the determinant and using the facts that $\operatorname{dim} H^{M}=g$ and $\operatorname{dim} H^{N}=$ $h$, we have

$$
\begin{aligned}
1 & =\left[\frac{\left(-n_{-}\left(v_{1}\right)+m_{+}\left(v_{1}\right), \ldots,-n_{-}\left(v_{2 g_{+}}\right)+m_{+}\left(v_{2 g_{+}}\right), m_{-}(x), n_{+}(y)\right)}{\left(\left(-n_{-}, m_{+}\right)(v), \text { lift of } k \text { to } H^{N} \oplus H^{M}\right)}\right] \\
& =\sum_{|P|=g-j} \varepsilon_{P}(-1)^{|\bar{P}|}\left[\frac{\left(m_{+}\left(v_{P}\right), n_{-}\left(v_{\bar{P}}\right), m_{-}(x), n_{+}(y)\right)}{\left(\left(-n_{-}, m_{+}\right)(v), \text { lift of } k \text { to } H^{N} \oplus H^{M}\right)}\right] \\
& =(-1)^{g(f+1)} \sum_{|P|=g-j} \varepsilon_{P}^{\prime}\left[\frac{\left(n_{-}\left(v_{\bar{P}}\right), n_{+}(y), m_{+}\left(v_{P}\right), m_{-}(x)\right)}{\left(\left(-n_{-}, m_{+}\right)(v), \text { lift of } k \text { to } H^{N} \oplus H^{M}\right)}\right] .
\end{aligned}
$$

Thus we obtain identity (4.11), up to multiplication by an element of $\pm G$ not depending on $j, x, y$. This concludes the proof of Lemma 4.4 .

\section{Back to the Alexander functor}

We show in this section that the functor $A$ is an instance of the functor $R$.

5.1. A formula for the Reidemeister function. Let $M$ be a compact connected orientable 3-manifold with connected boundary, and fix a base point $\star \in \partial M$. Let also $\varphi: H_{1}(M) \rightarrow G$ be a group homomorphism with values in a multiplicative subgroup $G$ of a field $\mathbb{F}$. We use the same notation as in $\S 4.1$, where we have introduced $\mathcal{R}_{M}^{\varphi}$.

When it does not vanish, the Reidemeister function $\mathcal{R}_{M}^{\varphi}$ is defined as an alternated product of 4 determinants since the $\mathbb{F}$-chain complex $C^{\varphi}(M, \star)$ has length 3 . We now give a recipe to compute it by means of a single determinant using Fox's free derivatives. We consider on this purpose a spine $X^{+}$of $M$, i.e. a 2-dimensional subcomplex $X^{+}$of a smooth triangulation of $M$ such that $M$ retracts to $X^{+}$by elementary collapses; we also assume that $\star$ is a vertex of $X^{+}$. (It is well known that any 3-manifold with boundary has a spine: see for instance [Mat03, Remark 1.1.5].) Next, we choose a maximal tree in the 1-skeleton of $X^{+}$which contains $\star$, and let $X$ be the 2-dimensional CW-complex obtained from $X^{+}$by collapsing that tree to the vertex $\star$. Hence $X$ has a single 0 -cell $\star$. We denote by $\gamma_{1}, \ldots, \gamma_{g+r}$ the 1-cells of $X$ and we denote by $R_{1}, \ldots, R_{r}$ the 2-cells of $X$; besides, each of these cells is given an arbitrary orientation. The fundamental group $\pi_{1}(\Gamma)=\pi_{1}(\Gamma, \star)$ of the 1-skeleton $\Gamma:=\gamma_{1} \cup \cdots \cup \gamma_{g+r}$ of $X$ is freely generated by the oriented loops $\gamma_{1}, \ldots, \gamma_{g+r}$, hence the free derivatives $\frac{\partial}{\partial \gamma_{1}}, \ldots, \frac{\partial}{\partial \gamma_{g+r}}: \mathbb{Z}\left[\pi_{1}(\Gamma)\right] \rightarrow$ $\mathbb{Z}\left[\pi_{1}(\Gamma)\right]$ are defined. Note that the attaching maps of the oriented 2 -cells $R_{1}, \ldots, R_{r}$ define some elements $\rho_{1}, \ldots, \rho_{r} \in \pi_{1}(\Gamma)$.

Lemma 5.1. Let $\kappa_{1}, \ldots, \kappa_{g}$ be oriented loops in $\Gamma$ based at $\star$ and, for all $i \in\{1, \ldots, g\}$, let $k_{i} \in H \simeq H_{1}^{\varphi}(X, \star)$ be the homology class of $1 \otimes \widehat{\kappa}_{i} \in C_{1}^{\varphi}(X, \star)$. Then

$$
\mathcal{R}_{M}^{\varphi}\left(k_{1} \wedge \cdots \wedge k_{g}\right)=\operatorname{det} \varphi i_{*}\left(\begin{array}{ccccc}
\frac{\partial \rho_{1}}{\partial \gamma_{1}} & \cdots & \cdots & \cdots & \frac{\partial \rho_{1}}{\partial \gamma_{g}+r} \\
\vdots & & & & \vdots \\
\frac{\partial \rho_{r}}{\partial \gamma_{1}} & \cdots & \ldots & \cdots & \frac{\partial \rho_{r}}{\partial \gamma_{g}+r} \\
\frac{\partial \kappa_{1}}{\partial \gamma_{1}} & \cdots & \cdots & \cdots & \frac{\partial \kappa_{1}}{\partial \gamma_{g}+r} \\
\vdots & & & & \vdots \\
\frac{\partial \kappa_{g}}{\partial \gamma_{1}} & \cdots & \cdots & \cdots & \frac{\partial \kappa_{g}}{\partial \gamma_{g}+r}
\end{array}\right) .
$$


Here the composition of $\varphi$ with the isomorphism $H_{1}(M) \simeq H_{1}(X)$ induced by the homotopy equivalence $M \simeq X$ is still denoted by $\varphi$, and the ring homomorphism $i_{*}$ : $\mathbb{Z}\left[\pi_{1}(\Gamma)\right] \rightarrow \mathbb{Z}\left[\pi_{1}(M)\right]$ is induced by the map $i: \Gamma \rightarrow M$ which is the inclusion $\Gamma \subset X$ composed with the homotopy equivalence $X \simeq M$.

Proof. The lemma is proved in a way similar to Milnor's result relating the Reidemeister torsion of a knot exterior to the Alexander polynomial of the knot [Mil62, Theorem 4]. (See also [Tur02, Theorem II.1.2].) By assumption, the pair $(M, \star)$ has the simple homotopy type of $\left(X^{+}, \star\right)$ and, using the multiplicativity property of torsions (Theorem A.3), it can be checked that the Reidemeister torsions of $(X, \star)$ and $\left(X^{+}, \star\right)$ are equal for any choice of homological bases. Therefore we can safely replace $M$ by $X$ in our computation of $\mathcal{R}_{M}^{\varphi}$. Thus we now consider the $\varphi$-twisted cell chain complex

$$
C:=C^{\varphi}(X, \star)=\mathbb{F} \otimes_{\mathbb{Z}\left[H_{1}(X)\right]} C\left(\widehat{X}, p_{X}^{-1}(\star)\right) .
$$

The lifts $\widehat{\gamma}_{1}, \ldots, \widehat{\gamma}_{g+r}$ of $\gamma_{1}, \ldots, \gamma_{g+r}$ define a basis $c_{1}:=\left(1 \otimes \widehat{\gamma}_{1}, \ldots, 1 \otimes \widehat{\gamma}_{g+r}\right)$ of $C$ in degree 1. Similarly, the lifts $\widehat{R}_{1}, \ldots, \widehat{R}_{r}$ of $R_{1}, \ldots, R_{r}$ that contain $\widehat{\star}$ define a basis $c_{2}:=\left(1 \otimes \widehat{R}_{1}, \ldots, 1 \otimes \widehat{R}_{r}\right)$ of $C$ in degree 2 .

Let $A^{\prime}$ be the square matrix with entries in $\mathbb{F}$ defined by the right-hand side of (5.1), and let $A$ be the $r \times(g+r)$ matrix defined by the first $r$ rows of $A^{\prime}$. Observe that $A$ is the matrix of $\partial_{2}: C_{2} \rightarrow C_{1}$ in the bases $c_{2}$ and $c_{1}$. Since $(X, \star)$ has no relative cells in degree $0, H \simeq H_{1}(C)$ is the cokernel of the linear map $\mathbb{F}^{r} \rightarrow \mathbb{F}^{g+r}$ defined by the multiplication $v \mapsto v A$. Assume that $\operatorname{dim} H>g$ : then the rank of $A$ is less than $r$, so that all the minors of $A$ of order $r$ vanish; by expanding the determinant of $A^{\prime}$ successively along its last $g$ rows, we obtain that $\operatorname{det} A^{\prime}=0$ and the lemma trivially holds true in that case. Therefore we can assume that $\operatorname{dim} H=g$.

Observe, next, that the last $g$ rows of $A^{\prime}$ give the vectors $k_{1}, \ldots, k_{g} \in H \simeq H_{1}^{\varphi}(X, \star)$ as linear combinations of the generators $\left[1 \otimes \widehat{\gamma}_{1}\right], \ldots,\left[1 \otimes \widehat{\gamma}_{g+r}\right]$ of $H_{1}^{\varphi}(X, \star) \simeq H$. If $k:=\left(k_{1}, \ldots, k_{g}\right)$ is not a basis of $H$, then $k_{1}, \ldots, k_{g}$ are linearly dependent: since the first $r$ rows of $A^{\prime}$ give a system of relations for the previous set of generators, we deduce that $\operatorname{det} A^{\prime}=0$ and the lemma is trivially true in that case too. Thus we can assume that $k$ is a basis of $H$. Let $c$ be the basis of $C$ given by $c_{1}$ in degree 1 and $c_{2}$ in degree 2 . By Lemma 4.1, the homology of $C$ is concentrated in degree 1 and, for all $i \in\{1, \ldots, g\}$, $1 \otimes \widehat{\kappa}_{i}$ is a 1-cycle of $C$ representing $k_{i} \in H \simeq H_{1}(C)$. So, by definition of the function $\mathcal{R}_{M}^{\varphi}$, we get

$$
\begin{aligned}
\mathcal{R}_{M}^{\varphi}\left(k_{1} \wedge \cdots \wedge k_{g}\right) & =\tau(C ; c, k) \\
& =\operatorname{det}\left(\text { matrix of }\left(\partial_{2}\left(c_{2}\right), 1 \otimes \widehat{\kappa}\right) \text { in the basis } c_{1}\right) .
\end{aligned}
$$

The conclusion follows from the previous two observations.

Remark 5.2. It follows from Lemma 5.1 that the Reidemeister function has the following integrality property: for all $h_{1}, \ldots, h_{g} \in H_{1}\left(M, \star ; \mathbb{Z}\left[H_{1}(M)\right]\right)$, we have

$$
\mathcal{R}_{M}^{\varphi}\left(\varphi_{*}\left(h_{1}\right) \wedge \cdots \wedge \varphi_{*}\left(h_{g}\right)\right) \in \varphi\left(\mathbb{Z}\left[H_{1}(M)\right]\right)
$$

where $\varphi_{*}: H_{1}\left(M, \star ; \mathbb{Z}\left[H_{1}(M)\right]\right) \rightarrow H_{1}^{\varphi}(M, \star)$ is the canonical map.

5.2. Specialization of $\mathrm{R}$ to $\mathrm{A}$. We now assume that $G$ is a finitely generated free abelian group, and we denote by $Q(G)$ the field of fractions of $\mathbb{Z}[G]$. Let $M$ be a compact connected orientable 3 -manifold with connected boundary, and fix a base point $\star \in \partial M$. Let $\varphi: H_{1}(M) \rightarrow G$ be a group homomorphism: we denote by $\varphi_{\mathbb{Z}}: \mathbb{Z}\left[H_{1}(M)\right] \rightarrow \mathbb{Z}[G]$ and by $\varphi: \mathbb{Z}\left[H_{1}(M)\right] \rightarrow Q(G)$ the extensions of $\varphi$ to ring homomorphisms. Set

$$
g:=g(M), \quad H_{\mathbb{Z}}:=H_{1}^{\varphi_{\mathbb{Z}}}(M, \star), \quad H:=H_{1}^{\varphi}(M, \star) .
$$


Lemma 5.3. We have the following commutative diagram:

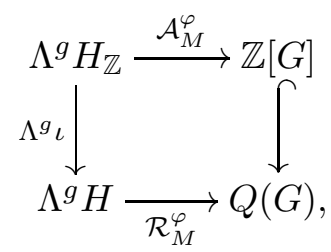

where $\iota: H_{\mathbb{Z}} \rightarrow H \simeq Q(G) \otimes_{\mathbb{Z}[G]} H_{\mathbb{Z}}$ denotes the canonical map.

Proof. We proceed as in $\S 5.1$ : we consider a spine $X^{+}$of $M$, and we obtain a 2dimensional $\mathrm{CW}$-complex $X$ with a single vertex $\star$ by collapsing a maximal tree in the 1-skeleton of $X^{+}$. The cells of $X$ are $\gamma_{1}, \ldots, \gamma_{g+r}$ in dimension 1 , and $R_{1}, \ldots, R_{r}$ in dimension 2. Orient $\gamma_{1}, \ldots, \gamma_{g+r}$ and $R_{1}, \ldots, R_{r}$ arbitrarily, and set

$$
C_{\mathbb{Z}}:=C^{\varphi_{\mathbb{Z}}}(X, \star), \quad C:=C^{\varphi}(X, \star)=Q(G) \otimes_{\mathbb{Z}[G]} C_{\mathbb{Z}} .
$$

Since $M$ deformation retracts to $X, H_{\mathbb{Z}}$ is isomorphic to $H_{1}^{\varphi_{\mathbb{Z}}}(X, \star)$ so that $H_{\mathbb{Z}}$ is the cokernel of $\partial_{2}: C_{\mathbb{Z}, 2} \rightarrow C_{\mathbb{Z}, 1}$. Let $\widehat{\gamma}_{1}, \ldots, \widehat{\gamma}_{g+r}$ be the preferred lifts of $\gamma_{1}, \ldots, \gamma_{g+r}$ to $\widehat{X}$, and let $\widehat{R}_{1}, \ldots, \widehat{R}_{r}$ be the lifts of $R_{1}, \ldots, R_{r}$ that contain $\widehat{\star}$ : we denote by $A$ the matrix of $\partial_{2}$ in the bases $\left(1 \otimes \widehat{R}_{1}, \ldots, 1 \otimes \widehat{R}_{r}\right)$ and $\left(1 \otimes \widehat{\gamma}_{1}, \ldots, 1 \otimes \widehat{\gamma}_{g+r}\right)$. This presentation matrix of the $\mathbb{Z}[G]$-module $H_{\mathbb{Z}}$ can be used to compute $\mathcal{A}_{M}^{\varphi}$. Specifically, let $k_{1}, \ldots, k_{g} \in H_{\mathbb{Z}}$ and assume that each $k_{i}$ has the form $\left[1 \otimes \widehat{\kappa}_{i}\right]$ where $\kappa_{i}$ is an oriented loop in the 1-skeleton of $X$ based at $\star$ : then $\mathcal{A}_{M}^{\varphi}\left(k_{1} \wedge \cdots \wedge k_{g}\right)$ is the determinant of the matrix obtained from $A$ by adding $g$ rows that express the vectors $1 \otimes \widehat{\kappa}_{1}, \ldots, 1 \otimes \widehat{\kappa}_{g}$ in the basis $\left(1 \otimes \widehat{\gamma}_{1}, \ldots, 1 \otimes \widehat{\gamma}_{g+r}\right)$ of $C_{\mathbb{Z}, 1}$. We deduce from formula (5.2) that $\mathcal{A}_{M}^{\varphi}\left(k_{1} \wedge \cdots \wedge k_{g}\right)=\mathcal{R}_{M}^{\varphi}\left(\iota\left(k_{1}\right) \wedge \cdots \wedge \iota\left(k_{g}\right)\right)$.

The next theorem, which compares the Alexander functor to the Reidemeister functor, is a direct application of Lemma 5.3.

Theorem 5.4. The following diagram is commutative:

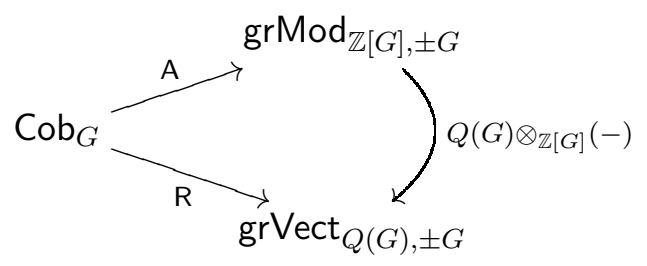

\section{REIDEMEISTER FUnCTOR AND KNOTS}

We now compute the functor $\mathrm{R}$ on knot exteriors and we consider, next, the situation of closed 3-manifolds. In this section, we fix a field $\mathbb{F}$ and a multiplicative subgroup $G$ of $\mathbb{F}$. The extension of a group homomorphism $\varphi: H \rightarrow G$ to a ring homomorphism $\mathbb{Z}[H] \rightarrow \mathbb{F}$ is still denoted by $\varphi$.

6.1. The abelian Reidemeister torsion of a $\mathbf{C W}$-pair. Let $(X, Y)$ be a pair of finite $\mathrm{CW}$-complexes, and let $\varphi: \mathbb{Z}\left[H_{1}(X)\right] \rightarrow \mathbb{F}$ be a ring homomorphism. We consider the $\varphi$-twisted cell chain complex $C^{\varphi}(X, Y)$ of the pair $(X, Y)$, which is a finite $\mathbb{F}$-chain complex of length $p:=\operatorname{dim} X$. For every $i \in\{0, \ldots, p\}$, let $n_{i} \geq 0$ be the number of relative $i$-cells of $(X, Y)$ and order them $\sigma_{1}^{(i)}, \ldots, \sigma_{n_{i}}^{(i)}$ in an arbitrary way. For every cell $\sigma$ of $(X, Y)$, we also choose an orientation of $\sigma$ and a lift $\hat{\sigma}$ of $\sigma$ to the maximal abelian cover $\widehat{X}$ of $X$. Thus, we obtain a basis $c:=\left(c_{p}, \ldots, c_{0}\right)$ of the $\mathbb{F}$-chain complex $C^{\varphi}(X, Y)$ 
where, for every $i \in\{0, \ldots, p\}$, the basis of $C_{i}^{\varphi}(X, Y)$ is $c_{i}:=\left(1 \otimes \hat{\sigma}_{1}^{(i)}, \ldots, 1 \otimes \hat{\sigma}_{n_{i}}^{(i)}\right)$. Recall that the Reidemeister torsion of the pair $(X, Y)$ with coefficients $\varphi$ is the scalar

$$
\tau^{\varphi}(X, Y):= \begin{cases}0 & \text { if } H^{\varphi}(X, Y) \neq 0 \\ \tau\left(C^{\varphi}(X, Y) ; c\right) & \text { if } H^{\varphi}(X, Y)=0\end{cases}
$$

where $\tau(C ; c)$ denotes the torsion of a finite acyclic $\mathbb{F}$-chain complex $C$ based by $c$ : see $\S$ A.1. The reader is referred to the monograph [Tur01] for an introduction to this combinatorial invariant. Without further structure on the $\mathrm{CW}$-pair $(X, Y)$, the scalar $\tau^{\varphi}(X, Y)$ is only defined up to multiplication by an element of $\pm \varphi\left(H_{1}(X)\right)$. If $Y=\varnothing$, we denote it by $\tau^{\varphi}(X)$.

6.2. The Reidemeister function in genus one. We now consider a compact connected orientable 3-manifold $M$ with connected boundary and a group homomorphism $\varphi: H_{1}(M) \rightarrow G$. Let $\star \in \partial M$ and set $H:=H_{1}^{\varphi}(M, \star)$. The next lemma relates the Reidemeister function $\mathcal{R}_{M}^{\varphi}$ to the Reidemeister torsion $\tau^{\varphi}(M)$ in genus one.

Lemma 6.1. Assume that $g(M)=1$ and that $\varphi$ is not trivial. Then, for any $k \in H$,

$$
\mathcal{R}_{M}^{\varphi}(k)=\tau^{\varphi}(M) \cdot \partial_{*}(k) .
$$

Here $\partial_{*}: H \rightarrow \mathbb{F}$ is the connecting homomorphism $H_{1}^{\varphi}(M, \star) \rightarrow H_{0}^{\varphi}(\star)$ in the long exact sequence of the pair $(M, \star)$, followed by the canonical isomorphism $H_{0}^{\varphi}(\star) \simeq \mathbb{F}$.

Proof. Consider a cell decomposition of $M$ where $\star$ is a 0 -cell. The short exact sequence of $\mathbb{F}$-chain complexes

$$
0 \longrightarrow \underbrace{C^{\varphi}(\star)}_{C^{\prime}:=} \longrightarrow \underbrace{C^{\varphi}(M)}_{C:=} \longrightarrow \underbrace{C^{\varphi}(M, \star)}_{C^{\prime \prime}:=} \longrightarrow 0
$$

induces the following long exact sequence in homology:

$$
\begin{gathered}
0 \longrightarrow 0 \longrightarrow 0 \longrightarrow 0 \longrightarrow H_{2}^{\varphi}(M) \longrightarrow H_{2}^{\varphi}(M, \star) \rightarrow \\
\rightarrow 0 \longrightarrow H_{1}^{\varphi}(M) \longrightarrow H_{1}^{\varphi}(M, \star) \stackrel{\partial_{*}}{\longrightarrow} H_{0}^{\varphi}(\star) \longrightarrow H_{0}^{\varphi}(M) \longrightarrow 0
\end{gathered}
$$

We regard (6.3) as an acyclic $\mathbb{F}$-chain complex $\mathcal{H}$ of length 12 : let $\left(h^{\prime}, h, h^{\prime \prime}\right)$ be the basis of $\mathcal{H}$ obtained by choosing bases $h^{\prime}, h, h^{\prime \prime}$ of $H\left(C^{\prime}\right), H(C), H\left(C^{\prime \prime}\right)$ in each degree. We choose an orientation and a lift to $\widehat{M}$ for every cell of $M$ and, for all $i \in\{0, \ldots, 3\}$, we order the $i$-cells in an arbitrary way. Thus, we obtain bases $c^{\prime}, c, c^{\prime \prime}$ of the complexes $C^{\prime}, C, C^{\prime \prime}$, respectively, which are compatible in the sense of $\S \mathrm{A} .1$. By the multiplicativity property of torsions (see Theorem A.3), we obtain

$$
\tau(C ; c, h)=\varepsilon \cdot \tau\left(C^{\prime} ; c^{\prime}, h^{\prime}\right) \cdot \tau\left(C^{\prime \prime} ; c^{\prime \prime}, h^{\prime \prime}\right) \cdot \tau\left(\mathcal{H} ;\left(h^{\prime}, h, h^{\prime \prime}\right)\right)
$$

where $\varepsilon$ is a sign independent of $h, h^{\prime}, h^{\prime \prime}$. If $H_{2}^{\varphi}(M) \neq 0$, then $\tau^{\varphi}(M)=0$ by definition, but (6.3) gives $H_{2}^{\varphi}(M, \star) \neq 0$ and Lemma 4.1 implies that $\operatorname{dim} H_{1}^{\varphi}(M, \star)>g(M)$ : hence $\mathcal{R}_{M}^{\varphi}=0$ by definition and (6.1) trivially holds true. Therefore we can assume that $H_{2}^{\varphi}(M)=0$.

Besides $H_{0}^{\varphi}(M)=0$ since $\varphi$ is non-trivial: the fact that $\chi(M)=1-g(M)$ is zero implies that $H_{1}^{\varphi}(M)=0$ as well. Thus the chain complex $\mathcal{H}$ defined by (6.3) is concentrated in degrees 2 and 3. Let $k \in H \backslash\{0\}$ which defines a basis $h^{\prime \prime}$ of $H\left(C^{\prime \prime}\right)$, and let $h^{\prime}$ be the basis of $H\left(C^{\prime}\right)$ defined by the canonical generator of $H_{0}^{\varphi}(\star)$. Then we obtain

$$
\tau\left(\mathcal{H} ;\left(h^{\prime}, h, h^{\prime \prime}\right)\right)=\left[\partial_{*}\left(h_{1}^{\prime \prime}\right) / h_{0}^{\prime}\right]^{(-1)^{2+1}}=\partial_{*}(k)^{-1} .
$$

Besides we have $\tau\left(C^{\prime} ; c^{\prime}, h^{\prime}\right)=1$ by our choices of $c^{\prime}$ and $h^{\prime}$. We conclude thanks to (6.4) that $\tau^{\varphi}(M)=\varepsilon \cdot \mathcal{R}_{M}^{\varphi}(k) \cdot \partial_{*}(k)^{-1}$. 
Remark 6.2. If $g(M)=0$ and $\varphi$ is not trivial, then $\mathcal{R}_{M}^{\varphi}: \mathbb{F}=\Lambda^{0} H \rightarrow \mathbb{F}$ is the zero map. Indeed, pick an oriented loop $\alpha$ in $M$ based at $\star$ such that $\varphi([\alpha]) \neq 1$; then $\partial_{*}: H \rightarrow \mathbb{F}$ does not vanish on $[\hat{\alpha}]$ and it follows that $\operatorname{dim} H>g(M)$.

6.3. The functor $\mathrm{R}$ on knot exteriors. Let $K$ be an oriented knot in a closed connected oriented 3-manifold $N$, and denote by $M_{K}$ the complement of an open tubular neighborhood of $K$ in $N$. We assume given a group homomorphism $\varphi_{K}: M_{K} \rightarrow G$ and an oriented closed curve $\lambda \subset \partial M_{K}$ such that $\varphi_{K}([\lambda]) \neq 1$. Thus the Reidemeister torsion $\tau^{\varphi_{K}}\left(M_{K}\right) \in \mathbb{F} / \pm G$ is defined.

We make $M_{K}$ a morphism $1 \rightarrow 0$ in the category Cob by choosing a boundaryparametrization $m: F(1,0) \rightarrow \partial M_{K}$, such that $\lambda_{-}:=m^{-1}(\lambda)$ is contained in the bottom surface $F_{1}$ and goes through the base point $\star$. Set $H_{-}:=H_{1}^{\varphi_{K} m_{-}}\left(F_{1}, \star\right)$. The following proposition, which is easily deduced from Lemma 6.1 , shows that the topological invariants $\tau^{\varphi_{K}}\left(M_{K}\right)$ and $\mathrm{R}\left(M_{K}, \varphi_{K}\right)$ are equivalent.

Proposition 6.3. With the above notation and for any $h \in \Lambda^{i} H_{-}$, we have

$$
\mathrm{R}\left(M_{K}, \varphi_{K}\right)(h)= \begin{cases}\tau^{\varphi_{K}}\left(M_{K}\right) \cdot \partial_{*}(h) & \text { if } i=1 \\ 0 & \text { otherwise }\end{cases}
$$

where $\partial_{*}: H_{-} \rightarrow \mathbb{F}$ is the connecting homomorphism for the pair $\left(F_{1}, \star\right)$. In particular, we have $\tau^{\varphi_{K}}\left(M_{K}\right)=\mathrm{R}\left(M_{K}, \varphi_{K}\right)\left(\left[\hat{\lambda}_{-}\right]\right) /\left(\varphi_{K}([\lambda])-1\right)$.

Example 6.4. If $G$ is the infinite cyclic group generated by $t, N$ is a homology 3sphere and $\varphi_{K}$ maps the oriented meridian $\mu$ of $K$ to $t$, then we know from [Mil62] that $\tau^{\varphi_{K}}\left(M_{K}\right)=\Delta(K) /(t-1)$. Thus we recover Proposition 3.3 by taking $\lambda:=\mu$.

6.4. The situation of closed 3-manifolds. Let $N$ be a closed connected orientable 3-manifold, and let $\varphi: H_{1}(N) \rightarrow G$ be a non-trivial group homomorphism. We wish to compute the Reidemeister torsion $\tau^{\varphi}(N)$ with coefficients $\varphi: \mathbb{Z}\left[H_{1}(N)\right] \rightarrow \mathbb{F}$ from the Reidemeister functor R. For this, we have to transform $N$ into a cobordism. Note that removing an open 3-ball $B$ from $N$ and regarding $N \backslash B$ as an element of $\operatorname{Cob}(0,0)$ is not fruitful, since the functor $\mathrm{R}$ maps this morphism to zero (see Remark 6.2).

We proceed in the following (rather indirect) way. Choose a knot $K \subset N$ such that $\varphi([K]) \neq 1$. Consider the complement $M_{K}$ of an open tubular neighborhood of $K$ in $N$, and fix a parallel $\rho \subset \partial M_{K}$ of $K$. Let $\varphi_{K}: H_{1}\left(M_{K}\right) \rightarrow G$ be the homomorphism obtained from $\varphi$ by restriction to $M_{K} \subset N$. Make $M_{K}$ a morphism $1 \rightarrow 0$ in Cob by choosing a boundary-parametrization $m: F(1,0) \rightarrow \partial M_{K}$ such that $\rho_{-}:=m^{-1}(\rho)$ is contained in the bottom surface $F_{1}$ and $\star \in \rho_{-}$.

Proposition 6.5. With the above notation, we have

$$
\tau^{\varphi}(N)=\frac{\mathrm{R}\left(M_{K}, \varphi_{K}\right)\left(\left[\widehat{\rho}_{-}\right]\right)}{(\varphi([K])-1)^{2}} \in \mathbb{F} / \pm G
$$

Proof. There is a formula describing (under certain circumstances) how the abelian Reidemeister torsion changes when a solid torus is glued along a 3-manifold with toroidal boundary: see [Tur02, §VII.1]. This formula applies to our situation and gives

$$
\tau^{\varphi_{K}}\left(M_{K}\right)=(\varphi([K])-1) \cdot \tau^{\varphi}(N) .
$$

We conclude by applying Proposition 6.3 to $\lambda:=\rho$.

As an application, we relate the functor A to the Alexander polynomial of closed 3 -manifolds. Thus, we now assume that $G$ is a finitely generated free abelian group and 
we take $\mathbb{F}:=Q(G)$. We consider the Alexander polynomial of $N$ with coefficients $\varphi$, namely

$$
\Delta^{\varphi}(N)=\Delta_{0} H_{1}^{\varphi} \mathbb{Z}(N) \in \mathbb{Z}[G] / \pm G
$$

where $\varphi_{\mathbb{Z}}: \mathbb{Z}\left[H_{1}(N)\right] \rightarrow \mathbb{Z}[G]$ is the extension of $\varphi: H_{1}(N) \rightarrow G$.

Proposition 6.6. With the above notation, we have

$$
\Delta^{\varphi}(N)= \begin{cases}\frac{\mathrm{A}\left(M_{K}, \varphi_{K}\right)\left(\left[\widehat{\rho}_{-}\right]\right)}{(\varphi([K])-1)^{2}} & \text { if } \operatorname{rank} \varphi\left(H_{1}(N)\right) \geq 2, \\ \frac{\mathrm{A}\left(M_{K}, \varphi_{K}\right)\left(\left[\widehat{\rho}_{-}\right]\right)}{\left(t^{n-1}+\cdots+t+1\right)^{2}} & \text { if } \operatorname{rank} \varphi\left(H_{1}(N)\right)=1 .\end{cases}
$$

In the second case, $t \in \varphi\left(H_{1}(N)\right)$ is a generator and $n \in \mathbb{N}$ is such that $\varphi([K])=t^{n}$.

Proof. Proposition 6.5 and Theorem 5.4 give

$$
\tau^{\varphi}(N)=\frac{\mathrm{R}\left(M_{K}, \varphi_{K}\right)\left(\left[\hat{\rho}_{-}\right]\right)}{(\varphi([K])-1)^{2}}=\frac{\mathrm{A}\left(M_{K}, \varphi_{K}\right)\left(\left[\widehat{\rho}_{-}\right]\right)}{(\varphi([K])-1)^{2}} \in Q(G) / \pm G .
$$

Besides, according to [Tur75], we have

$$
\tau^{\varphi}(N)= \begin{cases}\Delta^{\varphi}(N) & \text { if } \operatorname{rank} \varphi\left(H_{1}(N)\right) \geq 2 \\ \Delta^{\varphi}(N) /(t-1)^{2} & \text { if } \operatorname{rank} \varphi\left(H_{1}(N)\right)=1\end{cases}
$$

We conclude by combining (6.5) to (6.6).

\section{The MONOID OF HOMOLOGY COBORDISMS}

In this section, we fix an integer $k \geq 1$, an abelian group $G$ and a group homomorphism $\psi: H_{1}\left(F_{k}\right) \rightarrow G$. We shall compute the functors $\mathrm{A}$ and $\mathrm{R}$ on the monoid of homology cobordisms over the surface $F_{k}$.

7.1. Homology cobordisms. A homology cobordism over $F_{k}$ is a morphism $M: k \rightarrow k$ in the category Cob such that $m_{ \pm}: H_{1}\left(F_{k}\right) \rightarrow H_{1}(M)$ is an isomorphism. The set of equivalence classes of homology cobordisms defines a submonoid

$$
\mathcal{C}\left(F_{k}\right) \subset \operatorname{Cob}(k, k) \text {. }
$$

We restrict ourselves to homology cobordisms $M$ such that the composition

$$
H_{1}\left(F_{k}\right) \stackrel{m_{-}}{\simeq} H_{1}(M) \stackrel{m_{+}^{-1}}{\simeq} H_{1}\left(F_{k}\right) \stackrel{\psi}{\longrightarrow} G
$$

coincides with $\psi$. Thus we obtain a submonoid

$$
\mathcal{C}^{\psi}\left(F_{k}\right) \subset \mathcal{C}\left(F_{k}\right),
$$

which we also view as a submonoid of $\operatorname{Cob}_{G}((k, \psi),(k, \psi))$ by equipping every cobordism $M$ of the above form with the homomorphism $\psi:=\psi \circ m_{-}^{-1}=\psi \circ m_{+}^{-1}: H_{1}(M) \rightarrow G$.

Example 7.1. A homology cylinder is a homology cobordism $M$ over $F_{k}$ such that $m_{-}=m_{+}: H_{1}\left(F_{k}\right) \rightarrow H_{1}(M)$. Homology cylinders constitute a submonoid $\mathcal{I C}\left(F_{k}\right)$ of $\mathcal{C}\left(F_{k}\right)$ such that $\mathcal{I C}\left(F_{k}\right) \subset \mathcal{C}^{\psi}\left(F_{k}\right)$, whatever $\psi$ is. 
7.2. The Magnus representation. Assume now that $G$ is a multiplicative subgroup of a field $\mathbb{F}$. The extension of $\psi: H_{1}\left(F_{k}\right) \rightarrow G$ to a ring homomorphism $\mathbb{Z}\left[H_{1}\left(F_{k}\right)\right] \rightarrow \mathbb{F}$ is still denoted by $\psi$. We set

$$
H^{\psi}:=H_{1}^{\psi}\left(F_{k}, \star\right)
$$

and, when we are given an $M \in \mathcal{C}^{\psi}\left(F_{k}\right)$, we denote $H:=H_{1}^{\psi}(M, I)$. The fact that the map $m_{ \pm}: H_{1}\left(F_{k}\right) \rightarrow H_{1}(M)$ is an isomorphism of abelian groups implies that $m_{ \pm}: H^{\psi} \rightarrow H$ is an isomorphism of $\mathbb{F}$-vector spaces. (See [KLW01, Proposition 2.1] for a similar statement.) Consequently, we are allowed to set $r^{\psi}(M):=m_{+}^{-1} \circ m_{-}: H^{\psi} \rightarrow H^{\psi}$. This results in a monoid homomorphism

$$
r^{\psi}: \mathcal{C}^{\psi}\left(F_{k}\right) \longrightarrow \operatorname{Aut}\left(H^{\psi}\right),
$$

which is called the Magnus representation. See [Sak12] for a survey of this invariant.

7.3. The restriction of $\mathrm{R}$ to homology cobordisms. The Reidemeister functor restricts to a monoid homomorphism

$$
\mathrm{R}: \mathcal{C}^{\psi}\left(F_{k}\right) \longrightarrow \operatorname{grVect}_{\mathbb{F}, \pm G}\left(\Lambda H^{\psi}, \Lambda H^{\psi}\right) .
$$

We now compute this projective representation of the monoid $\mathcal{C}^{\psi}\left(F_{k}\right)$.

Proposition 7.2. For any $M \in \mathcal{C}^{\psi}\left(F_{k}\right)$ with top surface $\partial_{+} M$, we have

$$
\mathrm{R}(M, \psi)=\tau^{\psi}\left(M, \partial_{+} M\right) \cdot \Lambda\left(r^{\psi}(M)\right): \Lambda H^{\psi} \longrightarrow \Lambda H^{\psi}
$$

where $\tau^{\psi}\left(M, \partial_{+} M\right)$ is the Reidemeister torsion of $\left(M, \partial_{+} M\right)$ as defined in $\S 6.1$.

Proof. We shall prove a slightly more general statement: let $\psi_{ \pm}: H_{1}\left(F_{k}\right) \rightarrow G$ be any group homomorphisms and assume that $M \in \mathcal{C}\left(F_{k}\right)$ is a cobordism such that $\psi_{-} \circ m_{-}^{-1}=$ $\psi_{+} \circ m_{+}^{-1}: H_{1}(M) \rightarrow G$. Then we claim that

$$
\mathrm{R}(M, \psi)=\tau^{\psi}\left(M, \partial_{+} M\right) \cdot \Lambda\left(m_{+}^{-1} \circ m_{-}\right): \Lambda H_{-} \longrightarrow \Lambda H_{+}
$$

where $H_{ \pm}:=H_{1}^{\psi_{ \pm}}\left(F_{k}, \star\right)$ and $\psi:=\psi_{ \pm} \circ m_{ \pm}^{-1}$. (The proposition is the particular case where $\psi_{+}=\psi_{-}: H_{1}\left(F_{k}\right) \rightarrow G$.)

To prove this claim, we set $g:=g(M)=2 k, H:=H_{1}^{\psi}(M, I)$ and let $h=\left(h_{1}, \ldots, h_{g}\right)$ be a basis of $H$. In order to compute $\mathcal{R}_{M}^{\psi}\left(h_{1} \wedge \cdots \wedge h_{g}\right)$, we consider the short exact sequence of $\mathbb{F}$-chain complexes:

$$
0 \longrightarrow \underbrace{C^{\psi_{+}}\left(F_{k}, \star\right)}_{C^{\prime}:=} \stackrel{m_{+}}{\longrightarrow} \underbrace{C^{\psi}(M, \star)}_{C:=} \longrightarrow \underbrace{C^{\psi}\left(M, \partial_{+} M\right)}_{C^{\prime \prime}:=} \longrightarrow 0
$$

The complex $C^{\prime \prime}$ is acyclic while $C^{\prime}$ and $C$ have their homology concentrated in degree 1 . Therefore, the long exact sequence in homology $\mathcal{H}$ induced by $(7.2)$ is concentrated in degrees 4 and 5 where it reduces to the map $m_{+}: H_{+}=H_{1}\left(C^{\prime}\right) \rightarrow H_{1}(C)=H$.

There exists a wedge of circles $S_{1} \vee \cdots \vee S_{g}$ based at $\star$ onto which the surface $F_{k}$ retracts by elementary collapses. Let $h^{\prime}=\left(h_{1}^{\prime}, \ldots, h_{g}^{\prime}\right)$ be the basis of $H_{+}$obtained by lifting each of the loops $S_{1}, \ldots, S_{g}$ to the maximal abelian cover of $F_{k}$. Then we have

$$
\tau\left(C^{\prime} ; c^{\prime}, h^{\prime}\right) \in \pm G \subset \mathbb{F}
$$

for any choice of ordered/oriented lifts of the relative cells of $\left(F_{k}, \star\right)$ inducing a basis $c^{\prime}$ of $C^{\prime}$. Besides, by the multiplicativity property of torsions (see Theorem A.3), we have

$$
\tau(C ; c, h)=\varepsilon \cdot \tau\left(C^{\prime} ; c^{\prime}, h^{\prime}\right) \cdot \tau\left(C^{\prime \prime} ; c^{\prime \prime}\right) \cdot \tau\left(\mathcal{H} ;\left(h^{\prime}, h\right)\right) \in \mathbb{F} \backslash\{0\}
$$


for some appropriate choices of ordered/oriented lifts of the relative cells, which result in bases $c^{\prime}, c, c^{\prime \prime}$ of the chain complexes. Here $\varepsilon$ is a sign not depending on $h$ and $\mathcal{H}$ is regarded as an acyclic $\mathbb{F}$-chain complex based by $\left(h^{\prime}, h\right)$. We deduce that

$$
\begin{aligned}
\mathcal{R}_{M}^{\psi}\left(h_{1} \wedge \cdots \wedge h_{g}\right)=\tau(C ; c, h) & =\tau^{\psi}\left(M, \partial_{+} M\right) \cdot\left[m_{+}\left(h^{\prime}\right) / h\right]^{(-1)^{4+1}} \\
& =\tau^{\psi}\left(M, \partial_{+} M\right) \cdot\left[h / m_{+}\left(h^{\prime}\right)\right] .
\end{aligned}
$$

(Here the identities are up to multiplication by an element of $\pm G$ not depending on $h$.)

To proceed, we consider any integer $j \geq 0$ and any $x \in \Lambda^{j} H_{-}$. Let $\omega: \Lambda^{g} H_{+} \rightarrow \mathbb{F}$ be the volume form defined by $\omega\left(h_{1}^{\prime} \wedge \cdots \wedge h_{g}^{\prime}\right)=1$. (Note that $\omega$ is integral.) Then, for any $y \in \Lambda^{g-j} H_{+}$, we have

$$
\begin{aligned}
\omega(\mathrm{R}(M, \psi)(x) \wedge y) & =\mathcal{R}_{M}^{\psi}\left(\Lambda^{j} m_{-}(x) \wedge \Lambda^{g-j} m_{+}(y)\right) \\
& =\tau^{\psi}\left(M, \partial_{+} M\right) \cdot\left[\left(\Lambda^{j} m_{-}(x) \wedge \Lambda^{g-j} m_{+}(y)\right) / m_{+}\left(h^{\prime}\right)\right] \\
& =\tau^{\psi}\left(M, \partial_{+} M\right) \cdot\left[\left(\Lambda^{j}\left(m_{+}^{-1} m_{-}\right)(x) \wedge y\right) / h^{\prime}\right] \\
& =\tau^{\psi}\left(M, \partial_{+} M\right) \cdot \omega\left(\Lambda^{j}\left(m_{+}^{-1} m_{-}\right)(x) \wedge y\right) .
\end{aligned}
$$

We conclude that $\mathrm{R}(M, \psi)(x)=\tau^{\psi}\left(M, \partial_{+} M\right) \cdot \Lambda^{j}\left(m_{+}^{-1} m_{-}\right)(x)$ up to multiplication by an element of $\pm G$ not depending on $x$, which proves (7.1).

7.4. The restriction of A to homology cobordisms. Assume now that the abelian group $G$ is finitely generated and free, and assume that $\mathbb{F}:=Q(G)$. We denote by $\psi_{\mathbb{Z}}: \mathbb{Z}\left[H_{1}\left(F_{k}\right)\right] \rightarrow \mathbb{Z}[G]$ the extension of $\psi: H_{1}\left(F_{k}\right) \rightarrow G$ to a ring homomorphism and we set $H_{\mathbb{Z}}^{\psi}:=H_{1}^{\psi_{\mathbb{Z}}}\left(F_{k}, \star\right)$. The Alexander functor restricts to a monoid homomorphism

$$
\mathrm{A}: \mathcal{C}^{\psi}\left(F_{k}\right) \longrightarrow \operatorname{grMod}_{\mathbb{Z}[G], \pm G}\left(\Lambda H_{\mathbb{Z}}^{\psi}, \Lambda H_{\mathbb{Z}}^{\psi}\right) .
$$

This projective representation of the monoid $\mathcal{C}^{\psi}\left(F_{k}\right)$ is computed as follows.

Proposition 7.3. For any $M \in \mathcal{C}^{\psi}\left(F_{k}\right)$, we have the commutative diagram

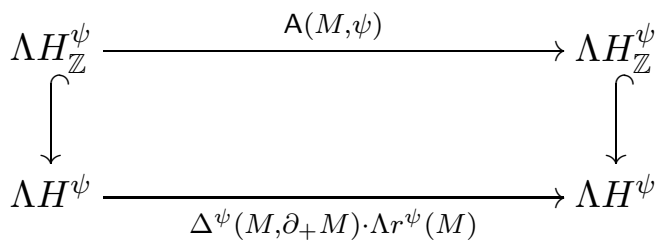

where $\Delta^{\psi}\left(M, \partial_{+} M\right)$ is the Alexander polynomial of the pair $\left(M, \partial_{+} M\right)$ as defined in $\S 3.1$.

Proof. The proposition can be proved directly from the definition of A, using an appropriate presentation of the $\mathbb{Z}[G]$-module $H_{1}^{\psi_{\mathbb{Z}}}(M, I)$. It also follows from Theorem 5.4, Proposition 7.2 and the fact that

$$
\tau^{\psi}\left(M, \partial_{+} M\right)=\Delta^{\psi}\left(M, \partial_{+} M\right) \in \mathbb{Z}[G] / \pm G .
$$

The latter identity is shown using the fact that $M$ collapses, relatively to $\partial_{+} M$, onto a cell complex having only 1-cells and 2-cells in an equal number. (For instance, consider the CW-complex resulting from a handle decomposition of $M$ as discussed in §8.1.) Thus, the computation of both invariants $\tau^{\psi}\left(M, \partial_{+} M\right)$ and $\Delta^{\psi}\left(M, \partial_{+} M\right)$ reduces to the determinant of a same matrix. (See [FJR11, Lemma 3.6] for instance.)

Example 7.4. Assume that $G:=\{1\}$ is the trivial group. Then $\mathcal{C}^{\psi}\left(F_{k}\right)=\mathcal{C}\left(F_{k}\right)$. Moreover $\mathbb{Z}[G]=\mathbb{Z}$ and $Q(G)=\mathbb{Q}$, so that $H_{\mathbb{Z}}^{\psi}=H_{1}\left(F_{k}\right)$ and $H^{\psi}=H_{1}\left(F_{k} ; \mathbb{Q}\right)$. Note that $\Delta^{\psi}\left(M, \partial_{+} M\right)=1$ since $H_{1}^{\psi_{\mathbb{Z}}}\left(M, \partial_{+} M\right)=H_{1}\left(M, \partial_{+} M\right)$ is trivial in that case. 
It follows from Proposition 7.3 that $\mathrm{A}(M, \psi): \Lambda H_{1}\left(F_{k}\right) \rightarrow \Lambda H_{1}\left(F_{k}\right)$ is induced by the isomorphism $\left(m_{+}\right)^{-1} m_{-}: H_{1}\left(F_{k}\right) \rightarrow H_{1}\left(F_{k}\right)$.

Remark 7.5. If two cobordisms $M, M^{\prime} \in \mathcal{C}^{\psi}\left(F_{k}\right)$ are homology cobordant, then we have $r^{\psi}(M)=r^{\psi}\left(M^{\prime}\right)\left(\right.$ see $\left[S a k 08\right.$, Theorem 3.6]), but it may happen that $\Delta^{\psi}\left(M, \partial_{+} M\right) \neq$ $\Delta^{\psi}\left(M^{\prime}, \partial_{+} M^{\prime}\right)$ (see [MM13, Lemma 3.15] for an example). It follows from Proposition 7.3 that the restriction of $\mathrm{A}$ to $\mathcal{C}^{\psi}\left(F_{k}\right)$ is stronger than the representation $r^{\psi}$.

\section{Computations with Heegaard splittings}

Let $G$ be a multiplicative subgroup of a field $\mathbb{F}$. We give a simple recipe to compute the functor $\mathrm{R}=\mathrm{R}_{\mathbb{F}, G}$ using Heegaard splittings of cobordisms. In this section, the extension of a group homomorphism $\rho: H \rightarrow G$ to a ring homomorphism $\mathbb{Z}[H] \rightarrow \mathbb{F}$ is still denoted by $\rho$.

8.1. Heegaard splittings. In order to obtain concrete formulas for the functor R, it is convenient to fix compatible systems of "meridians and parallels" on the model surfaces. Specifically, we choose on the model surface $F_{1}$ a meridian $\alpha$ and a parallel $\beta$, which means the following: $\alpha$ and $\beta$ are oriented simple closed curves in the interior of $F_{1}$ meeting transversely at a single point with homological intersection $[\alpha] \bullet[\beta]=+1$. Then the identification between $F_{1} \sharp_{\partial} \cdots \sharp_{\partial} F_{1}$ and $F_{k}$ induces, for any integer $k \geq 1$, a system of meridians and parallels $\left(\alpha_{1}, \ldots, \alpha_{k}, \beta_{1}, \ldots, \beta_{k}\right)$ on the surface $F_{k}$.

For any $k \geq 0$, we denote by $C_{0}^{k} \in \operatorname{Cob}(0, k)$ the cobordism obtained from $F_{k} \times[-1,1]$ by attaching $k 2$-handles along the curves $\alpha_{1} \times\{-1\}, \ldots, \alpha_{k} \times\{-1\}$. Similarly, let $C_{k}^{0} \in \operatorname{Cob}(k, 0)$ be the cobordism obtained from $F_{k} \times[-1,1]$ by attaching $k$ 2-handles along the curves $\beta_{1} \times\{1\}, \ldots, \beta_{k} \times\{1\}$. Observe that $C_{k}^{0} \circ C_{0}^{k}=C_{0}^{0} \in \operatorname{Cob}(0,0)$ is the 3-dimensional ball $F_{0} \times[-1,1]$. Thus we shall refer to $C_{k}^{0}$ and $C_{0}^{k}$ as the upper and lower handlebodies, respectively. (Clearly, these notions depend on the above choice of meridians and parallels.)

Let also $\mathcal{M}\left(F_{k}\right)$ be the mapping class group of the surface $F_{k}$, which consists of isotopy classes of (orientation-preserving) homeomorphisms $f: F_{k} \rightarrow F_{k}$ fixing $\partial F_{k}$ pointwisely. The mapping cylinder construction, which associates to any such homeomorphism $f$ the cobordism

$$
\mathbf{c}(f):=\left(F_{k} \times[-1,1],(f \times\{-1\}) \cup\left(\partial F_{k} \times \operatorname{Id}\right) \cup(\operatorname{Id} \times\{1\})\right),
$$

defines an embedding $\mathbf{c}: \mathcal{M}\left(F_{k}\right) \rightarrow \mathcal{C}\left(F_{k}\right)$ of the mapping class group into the monoid of homology cobordisms (see $\S 7.1$ ).

Let $M \in \operatorname{Cob}\left(g_{-}, g_{+}\right)$be an arbitrary cobordism. By elementary Morse theory, the 3-manifold underlying $M$ can be obtained from the trivial cobordism $F_{g_{+}} \times[-1,1]$ by attaching simultaneously some 1-handles (say, $r_{+} \geq 0$ ) along the "bottom surface" $F_{g_{+}} \times\{-1\}$, and by attaching subsequently some 2-handles (say, $r_{-} \geq 0$ ) along the new "bottom surface." We obtain in that way a Heegaard splitting of $M$, i.e. a decomposition in the monoidal category Cob of the form

$$
M=\left(C_{r_{+}}^{0} \otimes \operatorname{Id}_{g_{+}}\right) \circ \mathbf{c}(f) \circ\left(C_{0}^{r_{-}} \otimes \operatorname{Id}_{g_{-}}\right)
$$

where $g_{+}+r_{+}=g_{-}+r_{-}$and $f \in \mathcal{M}\left(F_{g_{ \pm}+r_{ \pm}}\right)$. See [Ker03b, Theorem 5].

8.2. Computation of $\mathrm{R}$ with Heegaard splittings. We now assume that the above cobordism $M$ comes with a group homomorphism $\varphi: H_{1}(M) \rightarrow G$ :

$$
(M, \varphi) \in \operatorname{Cob}_{G}\left(\left(g_{-}, \varphi_{-}\right),\left(g_{+}, \varphi_{+}\right)\right)
$$


The Heegaard splitting (8.1) of $M$ induces a decomposition in the monoidal category $\operatorname{Cob}_{G}$ by endowing each submanifold $S$ of that decomposition with the group homomorphism $\bar{\varphi}: H_{1}(S) \rightarrow G$ obtained by restricting $\varphi$ to $S \subset M$. Hence we obtain

$$
\mathrm{R}(M, \varphi)=\left(\mathrm{R}\left(C_{r_{+}}^{0}, \bar{\varphi}\right) \otimes \operatorname{Id}_{\Lambda H_{+}}\right) \circ \mathrm{R}(\mathbf{c}(f), \bar{\varphi}) \circ\left(\mathrm{R}\left(C_{0}^{r_{-}}, \bar{\varphi}\right) \otimes \operatorname{Id}_{\Lambda H_{-}}\right)
$$

where $H_{ \pm}:=H_{1}^{\varphi_{ \pm}^{ \pm}}\left(F_{g_{ \pm}}, \star\right)$ and the symbol $\bar{\varphi}$ denotes a representation in $G$ induced by $\varphi$. Thus the computation of $\mathrm{R}(M, \varphi)$ reduces to three cases: upper handlebodies, lower handlebodies and mapping cylinders.

To describe the values of $\mathrm{R}$ in those three cases, we need to fix further notation. Let $k \geq 0$ be an integer and let $\psi: H_{1}\left(F_{k}\right) \rightarrow G$ be a group homomorphism. We assume that, in our model surface $F_{1}$, the intersection point $\alpha \cap \beta$ is connected by an arc to the base point $\star \in \partial F_{1}$ : hence the curves $\alpha_{1}, \ldots, \alpha_{k}, \beta_{1}, \ldots, \beta_{k}$ are now viewed as oriented loops based at $\star \in \partial F_{k}$. We denote by $\left(a_{1}^{\psi}, \ldots, a_{k}^{\psi}, b_{1}^{\psi}, \ldots, b_{k}^{\psi}\right)$ the basis of $H_{1}^{\psi}\left(F_{k}, \star\right)$ obtained by lifting these loops to the maximal abelian cover:

$$
\forall i=1, \ldots, k, \quad a_{i}^{\psi}:=\left[1 \otimes \widehat{\alpha}_{i}\right], b_{i}^{\psi}:=\left[1 \otimes \widehat{\beta}_{i}\right] .
$$

Then the space $\Lambda H_{1}^{\psi}\left(F_{k}, \star\right)$ can be identified to $\Lambda A_{k}^{\psi} \otimes \Lambda B_{k}^{\psi}$ where $A_{k}^{\psi}:=\left\langle a_{1}^{\psi}, \ldots, a_{k}^{\psi}\right\rangle$ and $B_{k}^{\psi}:=\left\langle b_{1}^{\psi}, \ldots, b_{k}^{\psi}\right\rangle$ are the subspaces of $H_{1}^{\psi}\left(F_{k}, \star\right)$ corresponding to meridians and parallels, respectively.

Lemma 8.1. Let $\psi: H_{1}\left(C_{k}^{0}\right) \rightarrow G$ be a group homomorphism and let $\psi_{-}: H_{1}\left(F_{k}\right) \rightarrow G$ be the restriction of $\psi$ to $F_{k} \subset \partial C_{k}^{0}$. Then the linear map

$$
\mathrm{R}\left(C_{k}^{0}, \psi\right): \Lambda H_{1}^{\psi_{-}}\left(F_{k}, \star\right) \longrightarrow \mathbb{F}
$$

is trivial on $\Lambda^{i} A_{k}^{\psi_{-}} \otimes \Lambda^{j} B_{k}^{\psi_{-}}$if $i \neq k$ or $j \neq 0$, and it sends $a_{1}^{\psi_{-}} \wedge \cdots \wedge a_{k}^{\psi_{-}}$to 1.

Proof. Set $N:=C_{k}^{0} \in \operatorname{Cob}(k, 0)$. Since $\mathrm{R}(N, \psi)$ has degree $-k$, it must be trivial on $\Lambda^{r} H_{1}^{\psi_{-}}\left(F_{k}, \star\right)$ for $r \neq k$. It remains to compute

$$
\mathrm{R}(N, \psi)\left(x_{1} \wedge \cdots \wedge x_{k}\right)=\mathcal{R}_{N}^{\psi}\left(n_{-}\left(x_{1}\right) \wedge \cdots \wedge n_{-}\left(x_{k}\right)\right)
$$

for any $x_{1}, \ldots, x_{k} \in H_{1}^{\psi_{-}}\left(F_{k}, \star\right)$. If one of the $x_{i}$ 's belongs to $B_{k}^{\psi_{-}}$, the right-hand side of (8.3) is zero since, for all $j \in\{1, \ldots, k\}, \beta_{j}$ bounds a disk in $N$ so that $n_{-}\left(b_{j}^{\psi_{-}}\right)=0$. So, we can assume that $x_{1} \wedge \cdots \wedge x_{k}=a_{1}^{\psi_{-}} \wedge \cdots \wedge a_{k}^{\psi_{-}}$. In this case, we apply Lemma 5.1 to the obvious spine $X=X^{+}$of $N$ : the spine $X$ is a wedge of circles whose 1-cells $\gamma_{1}, \ldots, \gamma_{k}$ are obtained by "pushing" the curves $\alpha_{1}, \ldots, \alpha_{k}$ in the interior of $N$. We deduce that the right-hand side of (8.3) is equal to 1 .

Lemma 8.2. Let $\psi: H_{1}\left(C_{0}^{k}\right) \rightarrow G$ be a group homomorphism and let $\psi_{+}: H_{1}\left(F_{k}\right) \rightarrow G$ be the restriction of $\psi$ to $F_{k} \subset \partial C_{0}^{k}$. Then the linear map

$$
\mathrm{R}\left(C_{0}^{k}, \psi\right): \mathbb{F} \longrightarrow \Lambda H_{1}^{\psi_{+}}\left(F_{k}, \star\right)
$$

sends the scalar 1 to the multivector $a_{1}^{\psi_{+}} \wedge \cdots \wedge a_{k}^{\psi_{+}}$.

Proof. Set $\left(v_{1}, \ldots, v_{k}, v_{k+1}, \ldots, v_{2 k}\right):=\left(a_{1}^{\psi_{+}}, \ldots, a_{k}^{\psi_{+}}, b_{1}^{\psi_{+}}, \ldots, b_{k}^{\psi_{+}}\right)$and let $\omega$ be the volume form on $H_{1}^{\psi_{+}}\left(F_{k}, \star\right)$ defined by $\omega\left(v_{1} \wedge \cdots \wedge v_{2 k}\right)=1$. We denote $N:=C_{0}^{k} \in$ $\operatorname{Cob}(0, k)$ and write

$$
\mathrm{R}(N, \psi)(1)=\sum_{P} z_{P} \cdot v_{P} \in \Lambda^{k} H_{1}^{\psi_{+}}\left(F_{k}, \star\right)
$$


where $P$ runs over $k$-element subsets of $\{1, \ldots, 2 k\}$ and $v_{P}$ is the wedge of the $v_{p}$ 's for all $p \in P$. For any $k$-element subset $P \subset\{1, \ldots, 2 k\}$, we have

$$
\varepsilon_{P} \cdot z_{P}=\omega\left(\mathrm{R}(N, \psi)(1) \wedge v_{\bar{P}}\right)=\mathcal{R}_{N}^{\psi}\left(\Lambda^{k} n_{+}\left(v_{\bar{P}}\right)\right)
$$

where $\bar{P}$ is the complement of $P$ and $\varepsilon_{P}$ is the signature of the permutation $P \bar{P}$. To compute the right-hand side of (8.4), we apply Lemma 5.1 to the obvious spine $X=X^{+}$ of $N$ : the spine $X$ is a wedge of circles whose 1 -cells $\gamma_{1}, \ldots, \gamma_{k}$ are obtained by "pushing" the curves $\beta_{1}, \ldots, \beta_{k}$ in the interior of $N$. We obtain that $\mathcal{R}_{N}^{\psi}\left(\Lambda^{k} n_{+}\left(v_{\bar{P}}\right)\right)$ is trivial except if $\bar{P}=\{k+1, \ldots, 2 k\}$, in which case it takes the value 1 . We conclude that $z_{P}=1$ if $P=\{1, \ldots, k\}$ and $z_{P}=0$ otherwise.

Lemma 8.3. Let $f \in \mathcal{M}\left(F_{k}\right)$ and let $\psi_{ \pm}: H_{1}\left(F_{k}\right) \rightarrow G$ be group homomorphisms such that $\psi_{-}=\psi_{+} \circ f$. Denote by $\psi: H_{1}\left(F_{k} \times[-1,1]\right) \rightarrow G$ the isomorphism $\psi_{+} \circ$ pr, where $\operatorname{pr}: F_{k} \times[-1,1] \rightarrow F_{k}$ is the cartesian projection. Then

$$
\mathrm{R}(\mathbf{c}(f), \psi): \Lambda H_{1}^{\psi_{-}}\left(F_{k}, \star\right) \longrightarrow \Lambda H_{1}^{\psi_{+}}\left(F_{k}, \star\right)
$$

is induced by the isomorphism $f: H_{1}^{\psi_{-}}\left(F_{k}, \star\right) \rightarrow H_{1}^{\psi_{+}}\left(F_{k}, \star\right)$. Moreover, the matrix of this isomorphism in the bases $\left(a_{1}^{\psi_{ \pm}}, \ldots, a_{k}^{\psi_{ \pm}}, b_{1}^{\psi_{ \pm}}, \ldots, b_{k}^{\psi_{ \pm}}\right)$of $H_{1}^{\psi_{ \pm}}\left(F_{k}, \star\right)$ is

$$
\psi_{+}\left(\begin{array}{cccccc}
\frac{\partial f_{*}\left(\alpha_{1}\right)}{\partial \alpha_{1}} & \ldots & \frac{\partial f_{*}\left(\alpha_{k}\right)}{\partial \alpha_{1}} & \frac{\partial f_{*}\left(\beta_{1}\right)}{\partial \alpha_{1}} & \ldots & \frac{\partial f_{*}\left(\beta_{k}\right)}{\partial \alpha_{1}} \\
\vdots & & \vdots & \vdots & & \vdots \\
\frac{\partial f_{*}\left(\alpha_{1}\right)}{\partial \alpha_{k}} & \ldots & \frac{\partial f_{*}\left(\alpha_{k}\right)}{\partial \alpha_{k}} & \frac{\partial f_{*}\left(\beta_{1}\right)}{\partial \alpha_{k}} & \ldots & \frac{\partial f_{*}\left(\beta_{k}\right)}{\partial \alpha_{k}} \\
\frac{\partial f_{*}\left(\alpha_{1}\right)}{\partial \beta_{1}} & \ldots & \frac{\partial f_{*}\left(\alpha_{k}\right)}{\partial \beta_{1}} & \frac{\partial f_{*}\left(\beta_{1}\right)}{\partial \beta_{1}} & \ldots & \frac{\partial f_{*}\left(\beta_{k}\right)}{\partial \beta_{1}} \\
\vdots & & \vdots & \vdots & & \vdots \\
\frac{\partial f_{*}\left(\alpha_{1}\right)}{\partial \beta_{k}} & \ldots & \frac{\partial f_{*}\left(\alpha_{k}\right)}{\partial \beta_{k}} & \frac{\partial f_{*}\left(\beta_{1}\right)}{\partial \beta_{k}} & \ldots & \frac{\partial f_{*}\left(\beta_{k}\right)}{\partial \beta_{k}}
\end{array}\right)
$$

where $f_{*}: \pi_{1}\left(F_{k}, \star\right) \rightarrow \pi_{1}\left(F_{k}, \star\right)$ is induced by $f$.

Proof. The first statement follows from (7.1). The second statement is well known.

8.3. Computation of A with Heegaard splittings. Assume now that $G$ is a finitely generated free abelian group and take $\mathbb{F}:=Q(G)$. There are counterparts of Lemmas 8.1, 8.2 and 8.3 for the Alexander functor A. These counterparts follow from the same lemmas using Theorem 5.4, or they can be proved independently using presentations of $\mathbb{Z}[G]$-modules.

For $G=\{1\}$, we deduce that the functor $\mathrm{A}$ is essentially the same thing as the TQFT constructed in [FN91]. (Compare the formulas given in [Ker03a, §3] with the above lemmas.) However, there are a few technical differences: in particular, we have considered surfaces with circle boundary, whereas [FN91] works with closed surfaces.

\section{Duality}

We prove two duality properties for the Reidemeister functor. In this section, $\mathbb{F}$ is a field where a multiplicative subgroup $G$ is fixed, and we assume that $\mathbb{F}$ is equipped with an involutive automorphism $f \mapsto \bar{f}$ such that $\bar{g}=g^{-1}$ for all $g \in G$.

9.1. Twisted intersection form. The first duality satisfied by R involves the "twisted" intersection forms of oriented surfaces with boundary. We start by recalling this notion.

Let $k \geq 0$ be an integer and set $\pi:=\pi_{1}\left(F_{k}, \star\right)$. The homotopy intersection form of $F_{k}$ is the pairing $\lambda: \mathbb{Z}[\pi] \times \mathbb{Z}[\pi] \rightarrow \mathbb{Z}[\pi]$ defined by Turaev in [Tur78]. We also refer to Papakyriakopoulos' work [Pap75] where this form is implicit, and to Perron's work [Per06] where the same form $\lambda$ is re-discovered (and is denoted there by $\omega$ ). 
The twisted homology group $H_{1}\left(F_{k}, \star ; \mathbb{Z}[\pi]\right)$ is identified (as a left $\mathbb{Z}[\pi]$-module) to the augmentation ideal $I(\pi)$ of $\mathbb{Z}[\pi]$ in the following way: for any oriented loop $\gamma \subset F_{k}$ based at $\star$, let $\widetilde{\gamma}$ be the unique lift of $\gamma$ to the universal cover of $F_{k}$ starting at the preferred lift $\widetilde{\star}$, and identify $[1 \otimes \widetilde{\gamma}] \in H_{1}\left(F_{k}, \star ; \mathbb{Z}[\pi]\right)$ to $[\gamma]-1 \in I(\pi)$. Thus, by restricting $\lambda$ to $I(\pi) \times I(\pi)$, we obtain a pairing

$$
\langle-,-\rangle: H_{1}\left(F_{k}, \star ; \mathbb{Z}[\pi]\right) \times H_{1}\left(F_{k}, \star ; \mathbb{Z}[\pi]\right) \longrightarrow \mathbb{Z}[\pi] .
$$

The derivation properties of $\lambda$ given in [Tur78, Per06] imply that $\langle-,-\rangle$ is sesquilinear in the sense that

$$
\langle a x+y, z\rangle=a\langle x, z\rangle+\langle y, z\rangle, \quad\langle z, a x+y\rangle=\langle z, x\rangle S(a)+\langle z, y\rangle
$$

for all $a \in \mathbb{Z}[\pi]$ and $x, y, z \in H_{1}\left(F_{k}, \star ; \mathbb{Z}[\pi]\right)$. Here $S: \mathbb{Z}[\pi] \rightarrow \mathbb{Z}[\pi]$ is the antipode, i.e. the $\mathbb{Z}$-linear map defined by $S(a)=a^{-1}$ for all $a \in \pi$.

Let now $\psi: H_{1}\left(F_{k}\right) \rightarrow G$ be a group homomorphism: this induces a structure of right $\mathbb{Z}[\pi]$-module on $\mathbb{F}$. By identifying $H_{1}^{\psi}\left(F_{k}, \star\right)$ to $\mathbb{F} \otimes_{\mathbb{Z}[\pi]} H_{1}\left(F_{k}, \star ; \mathbb{Z}[\pi]\right)$, we obtain a pairing

$$
\langle-,-\rangle: H_{1}^{\psi}\left(F_{k}, \star\right) \times H_{1}^{\psi}\left(F_{k}, \star\right) \longrightarrow \mathbb{F}
$$

defined by $\left\langle f_{1} \otimes h_{1}, f_{2} \otimes h_{2}\right\rangle:=f_{1} \overline{f_{2}} \psi\left(\left\langle h_{1}, h_{2}\right\rangle\right)$ for all $f_{1}, f_{2} \in \mathbb{F}$ and $h_{1}, h_{2} \in H_{1}\left(F_{k}, \star ; \mathbb{Z}[\pi]\right)$. This pairing is sesquilinear in the sense that

$$
\langle f x+y, z\rangle=f\langle x, z\rangle+\langle y, z\rangle, \quad\langle z, f x+y\rangle=\bar{f}\langle z, x\rangle+\langle z, y\rangle
$$

for all $f \in \mathbb{F}$ and $x, y, z \in H_{1}^{\psi}\left(F_{k}, \star\right)$. The pairing (9.1) can also be defined using Poincaré duality (with twisted coefficients) and the fact that $H_{1}^{\psi}\left(F_{k}, J\right) \simeq H_{1}^{\psi}\left(F_{k}, \star\right) \simeq$ $H_{1}^{\psi}\left(F_{k}, J^{\prime}\right)$, where $J, J^{\prime}$ are two closed intervals such that $J \cup J^{\prime}=\partial F_{k}$ and $J \cap J^{\prime}=$ $\partial J=\partial J^{\prime}$. In particular, the pairing (9.1) is non-singular in the sense that $\langle x,-\rangle$ : $H_{1}^{\psi}\left(F_{k}, \star\right) \rightarrow \operatorname{Hom}\left(H_{1}^{\psi}\left(F_{k}, \star\right), \mathbb{F}\right)$ is an isomorphism for any $x \in H_{1}^{\psi}\left(F_{k}, \star\right)$.

For any integer $r \geq 1$, the pairing (9.1) also induces a non-singular sesquilinear pairing $\langle-,-\rangle: \Lambda^{r} H_{1}^{\psi}\left(F_{k}, \star\right) \times \Lambda^{r} H_{1}^{\psi}\left(F_{k}, \star\right) \rightarrow \mathbb{F}$ defined by

$$
\left\langle x_{1} \wedge \cdots \wedge x_{r}, y_{1} \wedge \cdots \wedge y_{r}\right\rangle=\operatorname{det}\left(\begin{array}{ccc}
\left\langle x_{1}, y_{1}\right\rangle & \cdots & \left\langle x_{1}, y_{r}\right\rangle \\
\vdots & \ddots & \vdots \\
\left\langle x_{r}, y_{1}\right\rangle & \cdots & \left\langle x_{r}, y_{r}\right\rangle
\end{array}\right)
$$

for all $x_{1}, \ldots, x_{r}, y_{1}, \ldots, y_{r} \in H_{1}^{\psi}\left(F_{k}, \star\right)$. For $r=0$, we set $\langle x, y\rangle:=x \bar{y}$ for all $x, y \in \mathbb{F}$.

Remark 9.1. The sesquilinear pairing (9.1) is not skew-hermitian. Instead, we have

$$
\forall x, y \in H_{1}^{\psi}\left(F_{k}, \star\right), \quad\langle x, y\rangle=-\overline{\langle y, x\rangle}+\partial_{*}(x) \overline{\partial_{*}(y)}
$$

where $\partial_{*}: H_{1}^{\psi}\left(F_{k}, \star\right) \rightarrow \mathbb{F}$ is the connecting homomorphism in the long exact sequence of the pair $\left(F_{k}, \star\right)$. This identity follows from a similar property for the homotopy intersection form $\lambda$ : see [Tur78, Per06].

9.2. First duality. Let $g_{-}, g_{+} \geq 0$ be integers. The $d u a l$ of an $M \in \operatorname{Cob}\left(g_{-}, g_{+}\right)$is the cobordism $\bar{M} \in \operatorname{Cob}\left(g_{+}, g_{-}\right)$obtained from $M$ by reversing its orientation and by composing its boundary-parametrization $m: F\left(g_{-}, g_{+}\right) \rightarrow \partial M$ with the orientationreversing homeomorphism

$$
\underbrace{-F_{g_{+}} \cup_{S^{1} \times\{-1\}}\left(S^{1} \times[-1,1]\right) \cup_{S^{1} \times\{1\}} F_{g_{-}}}_{F\left(g_{+}, g_{-}\right)} \stackrel{\cong}{\rightarrow} \underbrace{-F_{g_{-}} \cup_{S^{1} \times\{-1\}}\left(S^{1} \times[-1,1]\right) \cup_{S^{1} \times\{1\}} F_{g_{+}}}_{F\left(g_{-}, g_{+}\right)},
$$


which is given by "time-reversal" $(x, t) \mapsto(x,-t)$ on the annulus $S^{1} \times[-1,1]$ and by the identity on $F_{g_{+}}$and $F_{g_{-}}$.

Theorem 9.2. For any $(M, \varphi) \in \operatorname{Cob}_{G}\left(\left(g_{-}, \varphi_{-}\right),\left(g_{+}, \varphi_{+}\right)\right)$and for any $j \geq 0$, we have

$$
\forall x \in \Lambda^{j} H_{-}, \forall y \in \Lambda^{j+\delta g} H_{+}, \quad\langle\mathrm{R}(M, \varphi)(x), y\rangle=\langle x, \mathrm{R}(\bar{M}, \varphi)(y)\rangle
$$

where $\delta g:=g_{+}-g_{-}$and $H_{ \pm}:=H_{1}^{\varphi_{ \pm}}\left(F_{g_{ \pm}}, \star\right)$.

Of course, the identity (9.3) only holds true up to multiplication by a constant in $\pm G$ (independent of $x$ and $y$ ). The pairing $\langle-,-\rangle$ denotes the twisted intersection form of $H_{+}$(respectively, $H_{-}$) on the left-hand side (respectively, the right-hand side) of (9.3).

Proof of Theorem 9.2. Assume that $(M, \varphi)=\left(M^{\prime}, \varphi^{\prime}\right) \circ\left(M^{\prime \prime}, \varphi^{\prime \prime}\right)$ where $\left(M^{\prime}, \varphi^{\prime}\right)$ and $\left(M^{\prime \prime}, \varphi^{\prime \prime}\right)$ are two morphisms in $\operatorname{Cob}_{G}$ satisfying (9.3). Then the dual of $M$ is $\bar{M}^{\prime \prime} \circ \bar{M}^{\prime}$, and it easily follows that $(M, \varphi)$ also satisfies (9.3). Consequently, and following the discussion of $\S 8$, it is enough to prove (9.3) in the following three cases: (i) $M$ is a mapping cylinder; (ii) $M$ is a "stabilized" lower handlebody; (iii) $M$ is a "stabilized" upper handlebody.

Case (i). Assume that $g_{-}=g_{+}=: k$ and that $M=\mathbf{c}(f)$ is the mapping cylinder of an $f \in \mathcal{M}\left(F_{k}\right)$. Then $\bar{M}=\mathbf{c}\left(f^{-1}\right)$. Since $\varphi_{+} f=\varphi_{-}: H_{1}\left(F_{k}\right) \rightarrow G$ and since $f_{*}: \pi_{1}\left(F_{k}, \star\right) \rightarrow \pi_{1}\left(F_{k}, \star\right)$ preserves the homotopy intersection form, the isomorphism $f: H_{-} \rightarrow H_{+}$induced by $f: F_{k} \rightarrow F_{k}$ preserves the pairings (9.1). Using the first statement of Lemma 8.3, we obtain (9.3) as follows:

$$
\begin{aligned}
\forall x \in \Lambda^{j} H_{-}, \forall y \in \Lambda^{j} H_{+}, \quad\langle\mathrm{R}(M, \varphi)(x), y\rangle & =\left\langle\Lambda^{j} f(x), y\right\rangle \\
& =\left\langle x, \Lambda^{j} f^{-1}(y)\right\rangle=\langle x, \mathrm{R}(\bar{M}, \varphi)(y)\rangle .
\end{aligned}
$$

Interlude. In order to deal with cases (ii) and (iii), we need an explicit formula for the twisted intersection form $\langle-,-\rangle: H_{1}^{\psi}\left(F_{k}, \star\right) \times H_{1}^{\psi}\left(F_{k}, \star\right) \rightarrow \mathbb{F}$ defined by a group homomorphism $\psi: H_{1}\left(F_{k}\right) \rightarrow G$. For this, we fix a system of meridians and parallels $\left(\alpha_{1}, \ldots, \alpha_{k}, \beta_{1}, \ldots, \beta_{k}\right)$ on $F_{k}$ as explained in $\S 8.1$, and we denote by $\left(a_{1}^{\psi}, \ldots, a_{k}^{\psi}, b_{1}^{\psi}, \ldots, b_{k}^{\psi}\right)$ the corresponding basis of $H_{1}^{\psi}\left(F_{k}, \star\right)$ : see (8.2). For every $x, y \in$ $H_{1}\left(F_{k}\right)$, set $P^{\psi}(x, y):=(1-\psi(x)) \overline{(1-\psi(y))} \in \mathbb{F}$. Then, for an appropriate choice of meridians and parallels, the matrix of $\langle-,-\rangle$ in the basis $\left(a_{1}^{\psi}, \ldots, a_{k}^{\psi}, b_{1}^{\psi}, \ldots, b_{k}^{\psi}\right)$ is

$$
J^{\psi}=\left(\begin{array}{c|c}
J_{a a}^{\psi} & J_{a b}^{\psi} \\
\hline J_{b a}^{\psi} & J_{b b}^{\psi}
\end{array}\right)
$$

where $J_{a a}^{\psi}, J_{a b}^{\psi}, J_{b a}^{\psi}, J_{b b}^{\psi}$ are the following lower triangular matrices [Per06, Lemma 2.4]:

$$
\begin{aligned}
J_{a a}^{\psi}= & \left(\begin{array}{ccccc}
1-\psi\left(\alpha_{1}\right) & 0 & 0 & \ldots & 0 \\
P^{\psi}\left(\alpha_{2}, \alpha_{1}\right) & 1-\psi\left(\alpha_{2}\right) & 0 & \ldots & 0 \\
P^{\psi}\left(\alpha_{3}, \alpha_{1}\right) & P^{\psi}\left(\alpha_{3}, \alpha_{2}\right) & \ddots & \ddots & \vdots \\
\vdots & \vdots & \ddots & \ddots & 0 \\
P^{\psi}\left(\alpha_{k}, \alpha_{1}\right) & P^{\psi}\left(\alpha_{k}, \alpha_{2}\right) & \ldots & P^{\psi}\left(\alpha_{k}, \alpha_{k-1}\right) & 1-\psi\left(\alpha_{k}\right)
\end{array}\right), \\
J_{a b}^{\psi}= & \left(\begin{array}{ccccc}
\psi\left(\alpha_{1}\right) \overline{\psi\left(\beta_{1}\right)} & 0 & 0 & \ldots & 0 \\
P^{\psi}\left(\alpha_{2}, \beta_{1}\right) & \psi\left(\alpha_{2}\right) \overline{\psi\left(\beta_{2}\right)} & 0 & \ldots & 0 \\
P^{\psi}\left(\alpha_{3}, \beta_{1}\right) & P^{\psi}\left(\alpha_{3}, \beta_{2}\right) & \ddots & \ddots & \vdots \\
\vdots & \vdots & \ddots & \ddots & 0 \\
P^{\psi}\left(\alpha_{k}, \beta_{1}\right) & P^{\psi}\left(\alpha_{k}, \beta_{2}\right) & \ldots & P^{\psi}\left(\alpha_{k}, \beta_{k-1}\right) & \psi\left(\alpha_{k}\right) \overline{\psi\left(\beta_{k}\right)}
\end{array}\right),
\end{aligned}
$$




$$
\begin{gathered}
J_{b a}^{\psi}=\left(\begin{array}{cccccc}
1-\overline{\psi\left(\alpha_{1}\right)}-\psi\left(\beta_{1}\right) & 0 & 0 & \ldots & 0 \\
P^{\psi}\left(\beta_{2}, \alpha_{1}\right) & 1-\overline{\psi\left(\alpha_{2}\right)}-\psi\left(\beta_{2}\right) & 0 & \ldots & 0 \\
P^{\psi}\left(\beta_{3}, \alpha_{1}\right) & P^{\psi}\left(\beta_{3}, \alpha_{2}\right) & \ddots & \ddots & \vdots \\
\vdots & \vdots & \ddots & \ddots & 0 \\
P^{\psi}\left(\beta_{k}, \alpha_{1}\right) & P^{\psi}\left(\beta_{k}, \alpha_{2}\right) & \ldots & P^{\psi}\left(\beta_{k}, \alpha_{k-1}\right) & 1-\overline{\psi\left(\alpha_{k}\right)}-\psi\left(\beta_{k}\right)
\end{array}\right), \\
J_{b b}^{\psi}=\left(\begin{array}{ccccc}
1-\overline{\psi\left(\beta_{1}\right)} & 0 & 0 & \ldots & 0 \\
P^{\psi}\left(\beta_{2}, \beta_{1}\right) & 1-\overline{\psi\left(\beta_{2}\right)} & 0 & \ldots & 0 \\
P^{\psi}\left(\beta_{3}, \beta_{1}\right) & P^{\psi}\left(\beta_{3}, \beta_{2}\right) & \ddots & \ddots & \vdots \\
\vdots & \vdots & \ddots & \ddots & 0 \\
P^{\psi}\left(\beta_{k}, \beta_{1}\right) & P^{\psi}\left(\beta_{k}, \beta_{2}\right) & \ldots & P^{\psi}\left(\beta_{k}, \beta_{k-1}\right) & 1-\frac{0}{\psi\left(\beta_{k}\right)}
\end{array}\right)
\end{gathered}
$$

Besides, the following notation will be useful in the sequel. Let $\varepsilon \in\{+,-\}$ be a sign. We denote by $\left(v_{1}^{\varepsilon}, \ldots, v_{g_{\varepsilon}}^{\varepsilon}, v_{g_{\varepsilon}+1}^{\varepsilon}, \ldots, v_{2 g_{\varepsilon}}^{\varepsilon}\right):=\left(a_{1}^{\varphi_{\varepsilon}}, \ldots, a_{g_{\varepsilon}}^{\varphi_{\varepsilon}}, b_{1}^{\varphi_{\varepsilon}}, \ldots, b_{g_{\varepsilon}}^{\varphi_{\varepsilon}}\right)$ the basis of $H_{\varepsilon}=H_{1}^{\varphi_{\varepsilon}}\left(F_{g_{\varepsilon}}, \star\right)$. For any $s$-element subset $P \subset\left\{1, \ldots, 2 g_{\varepsilon}\right\}$, let $v_{P}^{\varepsilon} \in \Lambda^{s} H_{\varepsilon}$ be the wedge of the vectors $v_{p}^{\varepsilon}$ 's for all $p \in P$ and, when this makes sense, we shall also denote by $\left(v_{P}^{\varepsilon}\right)^{-\varepsilon} \in \Lambda^{s} H_{-\varepsilon}$ the multivector obtained from $v_{P}^{\varepsilon}$ by the transformations $a_{i}^{\varphi_{\varepsilon}} \mapsto a_{i-\varepsilon \delta g}^{\varphi_{-\varepsilon}}$ and $b_{i}^{\varphi_{\varepsilon}} \mapsto b_{i-\varepsilon \delta g}^{\varphi_{-\varepsilon}}$.

Case (ii). Assume that $M=C_{0}^{r} \otimes \operatorname{Id}_{g_{-}}$where $r=\delta g$. Note that $\varphi_{+}\left(\alpha_{i}\right)=1$ for all $i \in\{1, \ldots, r\}$, so that (9.4) and (9.5) applied to $\psi:=\varphi_{+}$give

$$
\forall i \in\{1, \ldots, r\}, \forall j \in\left\{1, \ldots, r+g_{-}\right\}, \quad\left\langle a_{i}^{\varphi_{+}}, a_{j}^{\varphi_{+}}\right\rangle=0,\left\langle a_{i}^{\varphi_{+}}, b_{j}^{\varphi_{+}}\right\rangle=\delta_{i j} \overline{\varphi_{+}\left(\beta_{j}\right)}
$$

and, combining this with (9.2), we also obtain

(9.9) $\forall i \in\{1, \ldots, r\}, \forall j \in\left\{1, \ldots, r+g_{-}\right\}, \quad\left\langle a_{j}^{\varphi_{+}}, a_{i}^{\varphi_{+}}\right\rangle=0,\left\langle b_{j}^{\varphi_{+}}, a_{i}^{\varphi_{+}}\right\rangle=-\delta_{i j} \varphi_{+}\left(\beta_{j}\right)$.

Let $P \subset\left\{1, \ldots, 2 g_{-}\right\}$with $|P|=j$ and let $Q \subset\left\{1, \ldots, 2 g_{+}\right\}$with $|Q|=r+j$. It follows from Lemma 8.2 that

$$
\left\langle\mathrm{R}(M, \varphi)\left(v_{P}^{-}\right), v_{Q}^{+}\right\rangle=\left\langle a_{1}^{\varphi_{+}} \wedge \cdots \wedge a_{r}^{\varphi_{+}} \wedge\left(v_{P}^{-}\right)^{+}, v_{Q}^{+}\right\rangle .
$$

According to (9.8), this determinant is zero if the subset $B:=\left\{g_{+}+1, \ldots, g_{+}+r\right\}$ is not contained in $Q$. If $B \subset Q$, then we get

$$
\begin{aligned}
& \left\langle\mathrm{R}(M, \varphi)\left(v_{P}^{-}\right), v_{Q}^{+}\right\rangle=\varepsilon_{B}\left\langle a_{1}^{\varphi_{+}} \wedge \cdots \wedge a_{r}^{\varphi_{+}} \wedge\left(v_{P}^{-}\right)^{+}, v_{B}^{+} \wedge v_{B^{c}}^{+}\right\rangle \\
& =\varepsilon_{B}\left\langle a_{1}^{\varphi_{+}} \wedge \cdots \wedge a_{r}^{\varphi_{+}}, v_{B}^{+}\right\rangle\left\langle\left(v_{P}^{-}\right)^{+}, v_{B^{c}}^{+}\right\rangle \\
& =\varepsilon_{B} \overline{\varphi_{+}\left(\beta_{1} \cdots \beta_{r}\right)}\left\langle\left(v_{P}^{-}\right)^{+}, v_{B^{c}}^{+}\right\rangle
\end{aligned}
$$

where $B^{c}:=Q \backslash B$ and $\varepsilon_{B}$ is the signature of the permutation $B B^{c}$ (where the elements of $B$ in increasing order are followed by the elements of $B^{c}$ in increasing order). We also deduce from (9.9) that $\left\langle\left(v_{P}^{-}\right)^{+}, v_{B^{c}}^{+}\right\rangle=0$ if $B^{c}$ has a non-empty intersection with $A:=\{1, \ldots, r\}$, and it follows that $\left\langle\mathrm{R}(M, \varphi)\left(v_{P}^{-}\right), v_{Q}^{+}\right\rangle=0$ if $A \cap Q \neq \varnothing$.

Besides, it follows from Lemma 8.1 that $\mathrm{R}(\bar{M}, \varphi)\left(v_{Q}^{+}\right)$is trivial if $A \cap Q \neq \varnothing$ or $B$ is not contained in $Q$. If $A \cap Q=\varnothing$ and $B \subset Q$, we get

$$
\left\langle v_{P}^{-}, \mathrm{R}(\bar{M}, \varphi)\left(v_{Q}^{+}\right)\right\rangle=\varepsilon_{B}\left\langle v_{P}^{-}, \mathrm{R}(\bar{M}, \varphi)\left(v_{B}^{+} \wedge v_{B^{c}}^{+}\right)\right\rangle=\varepsilon_{B}\left\langle v_{P}^{-},\left(v_{B^{c}}^{+}\right)^{-}\right\rangle .
$$

We deduce that $\left\langle\mathrm{R}(M, \varphi)\left(v_{P}^{-}\right), v_{Q}^{+}\right\rangle=\overline{\varphi_{+}\left(\beta_{1} \cdots \beta_{r}\right)}\left\langle v_{P}^{-}, \mathrm{R}(\bar{M}, \varphi)\left(v_{Q}^{+}\right)\right\rangle$for any $P, Q$. Since (9.3) is only required to hold true up to multiplication by a constant in $\pm G$, the theorem is proved in case (ii). 
Case (iii). Assume now that $M=C_{r}^{0} \otimes \operatorname{Id}_{g_{+}}$where $r=-\delta g$. Note that $\varphi_{-}\left(\beta_{i}\right)=1$ for all $i \in\{1, \ldots, r\}$, so that (9.7) and (9.5) applied to $\psi:=\varphi_{-}$give

$$
\forall i \in\left\{1, \ldots, r+g_{+}\right\}, \forall j \in\{1, \ldots, r\}, \quad\left\langle b_{i}^{\varphi_{-}}, b_{j}^{\varphi_{-}}\right\rangle=0,\left\langle a_{i}^{\varphi_{-}}, b_{j}^{\varphi_{-}}\right\rangle=\delta_{i j} \varphi_{-}\left(\alpha_{i}\right)
$$

and, combining this with (9.2), we also obtain

$$
\text { (9.11) } \forall i \in\left\{1, \ldots, r+g_{+}\right\}, j \in\{1, \ldots, r\}, \quad\left\langle b_{j}^{\varphi_{-}}, b_{i}^{\varphi_{-}}\right\rangle=0,\left\langle b_{j}^{\varphi_{-}}, a_{i}^{\varphi_{-}}\right\rangle=-\delta_{i j} \overline{\varphi_{-}\left(\alpha_{i}\right)}
$$

Let $P \subset\left\{1, \ldots, 2 g_{-}\right\}$with $|P|=j$ and let $Q \subset\left\{1, \ldots, 2 g_{+}\right\}$with $|Q|=j-r$. By Lemma 8.1, $\mathrm{R}(M, \varphi)\left(v_{P}^{-}\right)$is trivial if $P$ does not contain $A:=\{1, \ldots, r\}$ or $P$ has a non-empty intersection with $B:=\left\{g_{-}+1, \ldots, g_{-}+r\right\}$. If $A \subset P$ and $P \cap B=\varnothing$, we obtain

$$
\left\langle\mathrm{R}(M, \varphi)\left(v_{P}^{-}\right), v_{Q}^{+}\right\rangle=\varepsilon_{A}\left\langle\mathrm{R}(M, \varphi)\left(v_{A}^{-} \wedge v_{A^{c}}^{-}\right), v_{Q}^{+}\right\rangle=\varepsilon_{A}\left\langle\left(v_{A^{c}}^{-}\right)^{+}, v_{Q}^{+}\right\rangle
$$

where $A^{c}:=P \backslash A$ and $\varepsilon_{A}$ is the signature of the permutation $A A^{c}$.

Besides, Lemma 8.2 gives

$$
\left\langle v_{P}^{-}, \mathrm{R}(\bar{M}, \varphi)\left(v_{Q}^{+}\right)\right\rangle=\left\langle v_{P}^{-}, b_{1}^{\varphi_{-}} \wedge \cdots \wedge b_{r}^{\varphi_{-}} \wedge\left(v_{Q}^{+}\right)^{-}\right\rangle
$$

which, according to (9.10), is trivial if $P$ does not contain $A$. If $A \subset P$, we get

$$
\begin{aligned}
\left\langle v_{P}^{-}, \mathrm{R}(\bar{M}, \varphi)\left(v_{Q}^{+}\right)\right\rangle & =\varepsilon_{A}\left\langle v_{A}^{-} \wedge v_{A^{c}}^{-}, b_{1}^{\varphi_{-}} \wedge \cdots \wedge b_{r}^{\varphi_{-}} \wedge\left(v_{Q}^{+}\right)^{-}\right\rangle \\
& =\varepsilon_{A}\left\langle v_{A}^{-}, b_{1}^{\varphi_{-}} \wedge \cdots \wedge b_{r}^{\varphi_{-}}\right\rangle\left\langle v_{A^{c}}^{-},\left(v_{Q}^{+}\right)^{-}\right\rangle \\
& =\varepsilon_{A} \varphi_{-}\left(\alpha_{1} \cdots \alpha_{r}\right)\left\langle v_{A^{c}}^{-},\left(v_{Q}^{+}\right)^{-}\right\rangle .
\end{aligned}
$$

It follows from (9.11) that $\left\langle v_{A^{c}}^{-},\left(v_{Q}^{+}\right)^{-}\right\rangle=0$ if $A^{c}$ has a non-empty intersection with $B$, so that $\left\langle v_{P}^{-}, \mathrm{R}(\bar{M}, \varphi)\left(v_{Q}^{+}\right)\right\rangle=0$ if $P \cap B \neq \varnothing$. We deduce that $\left\langle\mathrm{R}(M, \varphi)\left(v_{P}^{-}\right), v_{Q}^{+}\right\rangle=$ $\overline{\varphi_{-}\left(\alpha_{1} \cdots \alpha_{r}\right)}\left\langle v_{P}^{-}, \mathrm{R}(\bar{M}, \varphi)\left(v_{Q}^{+}\right)\right\rangle$for any $P, Q$. This proves the theorem in case (iii).

Example 9.3. We consider the situation of $\S 7.3$ : let $\psi: H_{1}\left(F_{k}\right) \rightarrow G$ be a group homomorphism and let $M \in \mathcal{C}^{\psi}\left(F_{k}\right)$ with $k \geq 1$. According to Proposition $7.2, \mathrm{R}(M, \psi)$ is determined by the relative Reidemeister torsion $\tau^{\psi}\left(M, \partial_{+} M\right)$ and the Magnus representation $r^{\psi}(M): H^{\psi} \rightarrow H^{\psi}$, where $H^{\psi}:=H_{1}^{\psi}\left(F_{k}, \star\right)$. Specializing Theorem 9.2 to $j:=0$, we obtain the well-known duality theorem

$$
\tau^{\psi}\left(M, \partial_{+} M\right)=\overline{\tau^{\psi}\left(M, \partial_{-} M\right)} \in \mathbb{F} / \pm G
$$

see [Tur86, Appendix 3]. Next, specializing Theorem 9.2 successively to $j:=1$ and $j:=2$, we obtain the invariance property

$$
\forall x, z \in H^{\psi}, \quad\left\langle r^{\psi}(M)(x), r^{\psi}(M)(z)\right\rangle=\langle x, z\rangle,
$$

which is already observed in [Sak07, Theorem 2.4].

Example 9.4. We consider the situation of $\S 3.3$ : let $G$ be the infinite cyclic group generated by $t$ and $\mathbb{F}:=Q(G)$, let $M_{K}$ be the exterior of an oriented knot $K$ in an oriented homology 3-sphere and let $\varphi_{K}: H_{1}\left(M_{K}\right) \rightarrow G$ be the canonical isomorphism. There is a system of meridian and parallel $(\alpha, \beta)$ on $F_{1}$ and a boundary-parametrization $m: F(1,0) \rightarrow \partial M_{K}$ such that

(i) $m_{-}(\alpha)$ is the oriented meridian of $K$ and $m_{-}(\beta)$ is the parallel of $K$ that is null-homologous in $M_{K}$,

(ii) the matrix of $\langle-,-\rangle: H_{-} \times H_{-} \rightarrow \mathbb{F}$ in the corresponding basis $(a, b):=$ $\left(a_{1}^{\varphi_{K} m_{-}}, b_{1}^{\varphi_{K} m_{-}}\right)$of $H_{-}:=H_{1}^{\varphi_{K} m_{-}}\left(F_{1}, \star\right)$ is $\left(\begin{array}{cc}1-t & t \\ -t^{-1} & 0\end{array}\right)$. 
According to Proposition 3.3, the map $\mathrm{R}\left(M_{K}, \varphi_{K}\right)$ is determined by the Alexander polynomial $\Delta(K)$. By applying Theorem 9.2 successively to $x:=a$ and $x:=b$, we get

$$
\mathrm{R}\left(\overline{M_{K}}, \varphi_{K}\right)(1)=\overline{\Delta(K)} b \in H_{-} .
$$

9.3. Second duality. The second duality satisfied by $R$ does not involve the conjugation $f \mapsto \bar{f}$ of the field $\mathbb{F}$, and it is an immediate consequence of the definitions.

Proposition 9.5. For any $(M, \varphi) \in \operatorname{Cob}_{G}\left(\left(g_{-}, \varphi_{-}\right),\left(g_{+}, \varphi_{+}\right)\right)$and $j \geq 0$, we have

$$
\forall x \in \Lambda^{j} H_{-}, \forall y \in \Lambda^{g-j} H_{+}, \quad \omega(\mathrm{R}(M, \varphi)(x) \wedge y)=(-1)^{j g} \cdot \omega(x \wedge \mathrm{R}(\bar{M}, \varphi)(y))
$$

where $g:=g_{+}+g_{-}, H_{ \pm}:=H_{1}^{\varphi_{ \pm}}\left(F_{g_{ \pm}}, \star\right)$ and $\omega: \Lambda^{2 g_{ \pm}} H_{ \pm} \rightarrow \mathbb{F}$ is an arbitrary integral volume form.

Despite its simplicity, this proposition turns out to be interesting when it is combined with Theorem 9.2.

Example 9.6. We use the same notation as in Example 9.3. Let $\left(z_{1}, \ldots, z_{2 k}\right)$ be a basis of $H^{\psi}$ arising from of a basis of the free $\mathbb{Z}\left[H_{1}\left(F_{k}\right)\right]$-module $H_{1}\left(F_{k}, \star ; \mathbb{Z}\left[H_{1}\left(F_{k}\right)\right]\right)$ and assume that $\omega$ is given by $\omega\left(z_{1} \wedge \cdots \wedge z_{2 k}\right)=1$. By applying Proposition 9.5 to $x:=z_{1} \wedge \cdots \wedge z_{2 k}$, we get $\tau^{\psi}\left(M, \partial_{+} M\right) \cdot \operatorname{det} r^{\psi}(M)=\tau^{\psi}\left(M, \partial_{-} M\right)$. Combined with (9.12), this relation gives the symmetry

$$
\tau^{\psi}\left(M, \partial_{+} M\right) \cdot \operatorname{det} r^{\psi}(M)=\overline{\tau^{\psi}\left(M, \partial_{+} M\right)} \in \mathbb{F} / \pm G
$$

which is also observed in [Sak11, Theorem 5.3].

Example 9.7. We use the same notation as in Example 9.4. Let $\omega$ be the volume form on $H_{-}$defined by $\omega(a \wedge b)=1$. By applying Proposition 9.5 successively to $x:=a$ and $x:=b$, we obtain $\mathrm{R}\left(\overline{M_{K}}, \varphi_{K}\right)(1)=\Delta(K) b$. Combined with (9.13), we recover the classical symmetry of the Alexander polynomial:

$$
\Delta(K)=\overline{\Delta(K)} \in \mathbb{Z}[G] / \pm G .
$$

\section{Appendix A. A short REVIEW of COMBinatorial torsions}

We recall the definition and basic properties of the torsions of chain complexes. The reader is referred to [Mil66] and [Tur01] for further details and references. In this appendix, $\mathbb{F}$ is a field.

A.1. Definition of the torsion. Given an $\mathbb{F}$-vector space $V$ of finite dimension $n \geq 0$, an $n$-tuple $b=\left(b_{1}, \ldots, b_{n}\right)$ of vectors in $V$ and a basis $c=\left(c_{1}, \ldots, c_{n}\right)$ of $V$, we denote by $[b / c] \in \mathbb{F}$ the determinant of the matrix expressing $b$ in the basis $c$. Two bases $b$ and $c$ are said to be equivalent if $[b / c]=1$.

Given a short exact sequence of $\mathbb{F}$-vector spaces $0 \rightarrow V^{\prime} \rightarrow V \rightarrow V^{\prime \prime} \rightarrow 0$ and some bases $c^{\prime}$ and $c^{\prime \prime}$ of $V^{\prime}$ and $V^{\prime \prime}$ respectively, we denote by $c^{\prime} c^{\prime \prime}$ the equivalence class of bases of $V$ obtained by juxtaposing (in this order) the image of $c^{\prime}$ in $V$ and a lift of $c^{\prime \prime}$ to $V$.

By a finite $\mathbb{F}$-chain complex of length $m \geq 1$, we mean a chain complex $C$ in the category of finite-dimensional $\mathbb{F}$-vector spaces and we assume that $C$ is concentrated in degrees $0, \ldots, m$ :

$$
C=\left(C_{m} \stackrel{\partial_{m}}{\longrightarrow} C_{m-1} \longrightarrow \cdots \stackrel{\partial_{1}}{\longrightarrow} C_{0}\right) .
$$

A basis of $C$ is a family $c=\left(c_{m}, \ldots, c_{0}\right)$ where $c_{i}$ is a basis of $C_{i}$ for all $i \in\{0, \ldots, m\}$. A homological basis of $C$ is a family $h=\left(h_{m}, \ldots, h_{0}\right)$ where $h_{i}$ is a basis of the $i$-th homology group $H_{i}(C)$ for all $i \in\{0, \ldots, m\}$. If we have choosen a basis $b_{j}$ of the 
space of $j$-dimensional boundaries $B_{j}(C):=\operatorname{Im} \partial_{j+1}$ for all $j \in\{0, \ldots, m-1\}$, then a homological basis $h$ of $C$ induces an equivalence class of bases of $C_{i}$ for any $i$ : specifically, we consider the basis $\left(b_{i} h_{i}\right) b_{i-1}$ of $C_{i}$ obtained by juxtaposition in the following short exact sequences where we denote $Z_{i}(C):=\operatorname{Ker} \partial_{i}$ :

$$
\begin{aligned}
0 \longrightarrow B_{i}(C) \longrightarrow Z_{i}(C) \longrightarrow H_{i}(C) \longrightarrow 0 \\
\text { and } 0 \longrightarrow Z_{i}(C) \longrightarrow C_{i} \stackrel{\partial_{i}}{\longrightarrow} B_{i-1}(C) \longrightarrow 0
\end{aligned}
$$

Definition A.1. The torsion of a finite $\mathbb{F}$-chain complex $C$ of length $m$, equipped with a basis $c$ and a homological basis $h$, is the scalar

$$
\tau(C ; c, h):=\prod_{i=0}^{m}\left[\left(b_{i} h_{i}\right) b_{i-1} / c_{i}\right]^{(-1)^{i+1}} \in \mathbb{F} \backslash\{0\} .
$$

It is easily checked that this definition does not depend on the choice of $b_{0}, \ldots, b_{m}$ and, when $C$ is acyclic, we set $\tau(C ; c):=\tau(C ; c, \varnothing)$.

The following lemma, which is well known, is a way of viewing the torsion as a function in homology.

Lemma A.2. Let $C$ be a finite $\mathbb{F}$-chain complex of length $m \geq 1$, let $k \in\{0, \ldots, m\}$ and set $\beta:=\operatorname{dim} H_{k}(C)$. Assume given a basis $c=\left(c_{m}, \ldots, c_{0}\right)$ of $C$ and a basis $h_{i}$ of $H_{i}(C)$ for every $i \neq k$. Then there is a unique linear map $\ell: \Lambda^{\beta} H_{k}(C) \rightarrow \mathbb{F}$ such that

$$
\ell\left(v_{1} \wedge \cdots \wedge v_{\beta}\right)= \begin{cases}\tau\left(C ; c,\left(h_{m}, \ldots, h_{k+1}, v, h_{k-1}, \ldots, h_{0}\right)\right) & \text { if } k \text { is odd, } \\ \tau\left(C ; c,\left(h_{m}, \ldots, h_{k+1}, v, h_{k-1}, \ldots, h_{0}\right)\right)^{-1} & \text { if } k \text { is even },\end{cases}
$$

for any basis $v=\left(v_{1}, \ldots, v_{\beta}\right)$ of $H_{k}(C)$.

Proof. The unicity of $\ell$ is obvious and, clearly, we can assume that $k$ is odd. Let $s: H_{k}(C) \rightarrow Z_{k}(C)$ and $t: B_{k-1}(C) \rightarrow C_{k}$ be $\mathbb{F}$-linear sections of (A.1) and (A.2), respectively. For any $\beta$-tuple $v=\left(v_{1}, \ldots, v_{\beta}\right)$ of elements of $H_{k}(C)$, we set

$$
\ell(v):=\left[b_{k} s(v) t\left(b_{k-1}\right) / c_{k}\right] \cdot \prod_{i \neq k}\left[\left(b_{i} h_{i}\right) b_{i-1} / c_{i}\right]^{(-1)^{i+1}} \in \mathbb{F}
$$

where $b_{k} s(v) t\left(b_{k-1}\right)$ denotes the family of vectors of $C_{k}$ obtained by juxtaposing (in this order) $b_{k}, s(v)$ and $t\left(b_{k-1}\right)$. The resulting map $\ell: H_{k}(C)^{\beta} \rightarrow \mathbb{F}$ is multilinear and alternate, hence it induces a map $\ell: \Lambda^{\beta} H_{k}(C) \rightarrow \mathbb{F}$ with the desired property.

A.2. Multiplicativity of the torsion. Consider a short exact sequence of finite $\mathbb{F}$ chain complexes of length $m \geq 1$ :

$$
0 \longrightarrow C^{\prime} \longrightarrow C \longrightarrow C^{\prime \prime} \longrightarrow 0
$$

Let us assume that $C^{\prime}, C, C^{\prime \prime}$ are based by $c^{\prime}, c, c^{\prime \prime}$ respectively, and homologically based by $h^{\prime}, h, h^{\prime \prime}$ respectively. We further assume that the bases $c^{\prime}, c, c^{\prime \prime}$ are compatible in the sense that $c_{i}$ is equivalent to $c_{i}^{\prime} c_{i}^{\prime \prime}$ for every $i \in\{0, \ldots, m\}$. The short exact sequence (A.3) induces a long exact sequence in homology:

$$
\mathcal{H}:=\left(H_{m}\left(C^{\prime}\right) \rightarrow H_{m}(C) \rightarrow H_{m}\left(C^{\prime \prime}\right) \rightarrow \cdots \rightarrow H_{0}\left(C^{\prime}\right) \rightarrow H_{0}(C) \rightarrow H_{0}\left(C^{\prime \prime}\right)\right) .
$$

We regard $\mathcal{H}$ as an acyclic finite $\mathbb{F}$-chain complex based by

$$
\left(h^{\prime}, h, h^{\prime \prime}\right):=\left(h_{m}^{\prime}, h_{m}, h_{m}^{\prime \prime}, \ldots, h_{0}^{\prime}, h_{0}, h_{0}^{\prime \prime}\right) .
$$

The following formula is classical in the theory of combinatorial torsions: see [Mil66, Theorem 3.2] or [Tur86, Lemma 3.4.2]. 
Theorem A.3. With the above notation, we have

$$
\tau(C ; c, h)=\varepsilon \cdot \tau\left(C^{\prime} ; c^{\prime}, h^{\prime}\right) \cdot \tau\left(C^{\prime \prime} ; c^{\prime \prime}, h^{\prime \prime}\right) \cdot \tau\left(\mathcal{H} ;\left(h^{\prime}, h, h^{\prime \prime}\right)\right)
$$

where $\varepsilon$ is a sign depending only on the dimensions of the $\mathbb{F}$-vector spaces $C_{i}^{\prime}, C_{i}, C_{i}^{\prime \prime}$ and $H_{i}\left(C^{\prime}\right), H_{i}(C), H_{i}\left(C^{\prime \prime}\right)$ for all $i \in\{0, \ldots, m\}$.

Example A.4. Assume that $C=C^{\prime} \oplus C^{\prime \prime}$ and that the chain maps $C^{\prime} \rightarrow C$ and $C \rightarrow C^{\prime \prime}$ in (A.3) are the natural inclusion and projection, respectively. For all $i \in\{0, \ldots, m\}$, let $c_{i}$ be the basis of $C_{i}=C_{i}^{\prime} \oplus C_{i}^{\prime \prime}$ obtained by juxtaposing (in this order) some bases $c_{i}^{\prime}$ and $c_{i}^{\prime \prime}$ of $C_{i}^{\prime}$ and $C_{i}^{\prime \prime}$, respectively; similarly, let $h_{i}$ be the basis of $H_{i}(C)=H_{i}\left(C^{\prime}\right) \oplus H_{i}\left(C^{\prime \prime}\right)$ obtained by juxtaposing some bases $h_{i}^{\prime}$ and $h_{i}^{\prime \prime}$ of $H_{i}\left(C^{\prime}\right)$ and $H_{i}\left(C^{\prime \prime}\right)$, respectively. We set $c:=\left(c_{m}, \ldots, c_{0}\right)$ and $h:=\left(h_{m}, \ldots, h_{0}\right)$. Then $\tau(C ; c, h)=\varepsilon \cdot \tau\left(C^{\prime} ; c^{\prime}, h^{\prime}\right) \cdot \tau\left(C^{\prime \prime} ; c^{\prime \prime}, h^{\prime \prime}\right)$.

\section{REFERENCES}

[Arc10] Jana Archibald, The Multivariable Alexander Polynomial on Tangles, ProQuest LLC, Ann Arbor, MI, 2010, Thesis (Ph.D.)-University of Toronto (Canada).

[Ati88] Michael Atiyah, Topological quantum field theories, Inst. Hautes Études Sci. Publ. Math. (1988), no. 68, 175-186 (1989).

[BCF12] Stephen Bigelow, Alessia Cattabriga, and Vincent Florens, Alexander representation of tangles, arXiv:1203.4590, preprint 2012.

[Bla57] Richard C. Blanchfield, Intersection theory of manifolds with operators with applications to knot theory, Ann. of Math. (2) 65 (1957), 340-356.

[Cha74] T. A. Chapman, Topological invariance of Whitehead torsion, Amer. J. Math. 96 (1974), 488-497.

[Coh73] Marshall M. Cohen, A course in simple-homotopy theory, Springer-Verlag, New York-Berlin, 1973, Graduate Texts in Mathematics, Vol. 10.

[CT05] David Cimasoni and Vladimir Turaev, A Lagrangian representation of tangles, Topology 44 (2005), no. 4, 747-767.

[CT06] A L L L L L

[CY99] Louis Crane and David Yetter, On algebraic structures implicit in topological quantum field theories, J. Knot Theory Ramifications 8 (1999), no. 2, 125-163.

[FJR11] Stefan Friedl, András Juhász, and Jacob Rasmussen, The decategorification of sutured Floer homology, J. Topol. 4 (2011), no. 2, 431-478.

[FN91] Charles Frohman and Andrew Nicas, The Alexander polynomial via topological quantum field theory, Differential geometry, global analysis, and topology (Halifax, NS, 1990), CMS Conf. Proc., vol. 12, Amer. Math. Soc., Providence, RI, 1991, pp. 27-40.

[FN94] _ An intersection homology invariant for knots in a rational homology 3-sphere, Topology 33 (1994), no. 1, 123-158.

[Hab06] Kazuo Habiro, Bottom tangles and universal invariants, Algebr. Geom. Topol. 6 (2006), 11131214 (electronic).

[Ker03a] Thomas Kerler, Homology TQFT's and the Alexander-Reidemeister invariant of 3-manifolds via Hopf algebras and skein theory, Canad. J. Math. 55 (2003), no. 4, 766-821.

[Ker03b] _ Towards an algebraic characterization of 3-dimensional cobordisms, Diagrammatic morphisms and applications (San Francisco, CA, 2000), Contemp. Math., vol. 318, Amer. Math. Soc., Providence, RI, 2003, pp. 141-173.

[KLW01] Paul Kirk, Charles Livingston, and Zhenghan Wang, The Gassner representation for string links, Commun. Contemp. Math. 3 (2001), no. 1, 87-136.

[LD92] J.-Y. Le Dimet, Enlacements d'intervalles et représentation de Gassner, Comment. Math. Helv. 67 (1992), no. 2, 306-315.

[Les98] Christine Lescop, A sum formula for the Casson-Walker invariant, Invent. Math. 133 (1998), no. 3, 613-681.

[Mat03] Sergei Matveev, Algorithmic topology and classification of 3-manifolds, Algorithms and Computation in Mathematics, vol. 9, Springer-Verlag, Berlin, 2003.

[Mil62] John Milnor, A duality theorem for Reidemeister torsion, Ann. of Math. (2) 76 (1962), 137147.

[Mil66] J. Milnor, Whitehead torsion, Bull. Amer. Math. Soc. 72 (1966), 358-426. 
[MM13] Gwénaël Massuyeau and Jean-Baptiste Meilhan, Equivalence relations for homology cylinders and the core of the Casson invariant, Trans. Amer. Math. Soc. 365 (2013), no. 10, 5431-5502.

[Pap75] C. D. Papakyriakopoulos, Planar regular coverings of orientable closed surfaces, Knots, groups, and 3-manifolds (Papers dedicated to the memory of R. H. Fox), Princeton Univ. Press, Princeton, N.J., 1975, pp. 261-292. Ann. of Math. Studies, No. 84.

[Per06] Bernard Perron, A homotopic intersection theory on surfaces: applications to mapping class group and braids, Enseign. Math. (2) 52 (2006), no. 1-2, 159-186.

[Sak07] Takuya Sakasai, The symplecticity of the Magnus representation for homology cobordisms of surfaces, Bull. Austral. Math. Soc. 76 (2007), no. 3, 421-431.

[Sak08] _ The Magnus representation and higher-order Alexander invariants for homology cobordisms of surfaces, Algebr. Geom. Topol. 8 (2008), no. 2, 803-848.

[Sak11] , The Magnus representation and homology cobordism groups of homology cylinders, arXiv:1108.6111, preprint 2011.

[Sak12] _ A survey of Magnus representations for mapping class groups and homology cobordisms of surfaces, Handbook of Teichmüller theory. Volume III, IRMA Lect. Math. Theor. Phys., vol. 17, Eur. Math. Soc., Zürich, 2012, pp. 531-594.

[Tur75] Vladimir Turaev, The Alexander polynomial of a three-dimensional manifold, Mat. Sb. (N.S.) 97(139) (1975), no. 3(7), 341-359, 463.

[Tur78] V. G. Turaev, Intersections of loops in two-dimensional manifolds, Mat. Sb. 106(148) (1978), no. $4,566-588$.

[Tur86] Vladimir Turaev, Reidemeister torsion in knot theory, Uspekhi Mat. Nauk 41 (1986), no. 1(247), 97-147, 240.

[Tur89] _ Euler structures, nonsingular vector fields, and Reidemeister-type torsions, Izv. Akad. Nauk SSSR Ser. Mat. 53 (1989), no. 3, 607-643, 672.

[Tur01] Introduction to combinatorial torsions, Lectures in Mathematics ETH Zürich, Birkhäuser Verlag, Basel, 2001, Notes taken by Felix Schlenk.

[Tur02] _ Torsions of 3-dimensional manifolds, Progress in Mathematics, vol. 208, Birkhäuser Verlag, Basel, 2002.

[Tur10] Homotopy quantum field theory, EMS Tracts in Mathematics, vol. 10, European Mathematical Society (EMS), Zürich, 2010, Appendix 5 by Michael Müger and Appendices 6 and 7 by Alexis Virelizier.

VINCENT FLORENS

LMA, Université De PAU \& CNRS

Avenue De L'Université

64000 PAU, France

vincent.florens@univ-pau.fr

GWÉNAËL MASSUYEAU

IRMA, Université de Strasbourg \& CNRS

7 RUE RENÉ Descartes

67084 Strasbourg, France

massuyeau@math.unistra.fr 